\title{
Nonlinear electroelastic deformations of dielectric elastomer composites: II - Non-Gaussian elastic dielectrics
}

\author{
Victor Lefèvre, Oscar Lopez-Pamies \\ Department of Civil and Environmental Engineering, University of Illinois, Urbana-Champaign, IL 61801, USA
}

\begin{abstract}
This paper presents an analytical framework to construct approximate homogenization solutions for the macroscopic elastic dielectric response — under finite deformations and finite electric fields — of dielectric elastomer composites with two-phase isotropic particulate microstructures. The central idea consists in employing the homogenization solution derived in Part I of this work for ideal elastic dielectric composites within the context of a nonlinear comparison medium method - this is derived as an extension of the comparison medium method of Lopez-Pamies et al. (2013) in nonlinear elastostatics to the coupled realm of nonlinear electroelastostatics — to generate in turn a corresponding solution for composite materials with non-ideal elastic dielectric constituents. Complementary to this analytical framework, a hybrid finite-element formulation to construct homogenization solutions numerically in three dimensions is also presented.

The proposed analytical framework is utilized to work out a general approximate homogenization solution for non-Gaussian dielectric elastomers filled with nonlinear elastic dielectric particles that may exhibit polarization saturation. The solution applies to arbitrary (non-percolative) isotropic distributions of filler particles. By construction, it is exact in the limit of small deformations and moderate electric fields. For finite deformations and finite electric fields, its accuracy is demonstrated by means of direct comparisons with finite-element solutions. Aimed at gaining physical insight into the extreme enhancement in electrostriction properties displayed by emerging dielectric elastomer composites, various cases wherein the filler particles are of poly- and mono-disperse sizes and exhibit different types of elastic dielectric behavior are discussed in detail. Contrary to an initial conjecture in the literature, it is found (inter alia) that the isotropic addition of a small volume fraction of stiff (semi-)conducting/high-permittivity particles to dielectric elastomers does not lead to the extreme electrostriction enhancements observed in experiments. It is posited that such extreme enhancements are the manifestation of interphasial phenomena.
\end{abstract}

Key words: Comparison medium methods; Crouzeix-Raviart conforming elements; Microstructures; Electroactive materials; Electrostriction

\section{Introduction and main analytical result}

In Part I of this work (Lefèvre and Lopez-Pamies, 2016), we determined a general approximate homogenization solution for the macroscopic elastic dielectric response of Gaussian dielectric elastomers filled with any type of non-percolative isotropic distribution of ideal elastic dielectric particles. In this Part II of the work, we make use of this fundamental result as a building block to construct in turn a general approximate analytical solution for the macroscopic response of isotropic dielectric elastomer composites with non-ideal elastic dielectric constituents. This is accomplished by means of a nonlinear comparison medium method.

We recall that comparison medium methods are analytical techniques that allow one to construct variational approximations for the macroscopic properties of a given composite material in terms of the

Email addresses: vlefevre@illinois.edu (Victor Lefèvre), pamies@illinois.edu (Oscar Lopez-Pamies) 
macroscopic properties of another composite material. The latter is referred to as the comparison medium. The central defining idea of these methods can be traced back ${ }^{1}$ to the pioneering work of Talbot and Willis (1985) who laid out a complete comparison medium formalism — where the comparison medium is of arbitrary choice, possibly nonlinear and heterogeneous - for properties characterized by convex potentials of a single field. By introducing suitable partial Legendre transforms, Lopez-Pamies et al. (2013) extended the general formalism of Talbot and Willis (1985) to nonlinear properties characterized by the class of non-convex potentials that are inherent to nonlinear elasticity. In this paper, we work out an extension of the comparison medium method of Lopez-Pamies et al. (2013) to the coupled realm of nonlinear electroelastostatics, where the properties of interest are characterized by non-convex potentials that are functions of two (one mechanical and one electrical) fields. With suitably selected ideal elastic dielectric composites as choices for the comparison medium, we then employ this new formulation to construct a general approximate homogenization solution for the macroscopic elastic dielectric response of nonGaussian dielectric elastomers filled with nonlinear elastic dielectric particles that may exhibit polarization saturation. The solution is valid for any non-percolative two-phase isotropic particulate microstructure of choice. Aimed at scrutinizing experimental results for emerging dielectric elastomer composites, we work out and discuss in detail its specialization to two specific types of microstructures: $i$ ) an isotropic iterative microstructure wherein the particles are infinitely polydisperse in size and $i i$ ) an isotropic distribution of monodisperse spherical particles.

Complementary to the analytical approach outlined above, we also present in this paper a hybrid finiteelement (FE) formulation to construct homogenization solutions numerically for the macroscopic elastic dielectric response of isotropic dielectric elastomer composites. These are viewed as infinite periodic media where their defining repeated unit cells contain a large but finite number of particles that are randomly distributed (as dictated by a sequential adsorption algorithm) so as to approximate their isotropy. The independent variables in the conforming hybrid FE formulation that we adopt are taken as the displacement field, the electric potential, and a pressure field which allow for the analysis of the incompressible behaviors of the matrix and filler particles of interest in this work. The nonlinear algebraic equations that result from the FE discretization are solved by means of an arc-length method in order to be able to carry calculations passed the electromechanical limit loads inherent to nonlinear electroelastostatics. Also in complement to the analytical results to be worked out from the comparison medium method, we work out and discuss in detail FE solutions for two types of microstructures: $i$ ) an isotropic distribution of polydisperse spherical particles of a finite number of different sizes and $i$ ) an isotropic distribution of monodisperse spherical particles.

The presentation of the work is organized as follows. Section 2 introduces some basic notation and formulates the problem that defines the macroscopic response of non-Gaussian dielectric elastomers, filled with an arbitrary isotropic distribution of nonlinear elastic dielectric particles, under finite deformations and finite electric fields. Section 3 presents the comparison medium method for nonlinear electroelastostatics in its general form. Its application to the class of dielectric elastomer composites described in Section 2, which constitutes the main analytical result of this paper, is presented in Section 4. For convenience, we record this main result here:

The macroscopic elastic dielectric response of a non-Gaussian dielectric elastomer with (incompressible) free-energy function $W_{\mathrm{m}}=\Psi\left(I_{1}\right)-\varepsilon / 2 I_{5}^{E}$, filled with any type of isotropic distribution of nonlinear elastic dielectric particles with (incompressible) free-energy function $W_{\mathrm{p}}=\mu_{\mathrm{p}} / 2\left[I_{1}-3\right]-\mathcal{S}\left(I_{5}^{E}\right)$ at volume fraction $c$, is characterized by the (incompressible) effective free-energy function

$$
\bar{W}=(1-c) \Psi\left(\mathcal{I}_{1}\right)-\frac{(1-c) \mu_{0}}{2}\left[\mathcal{I}_{1}-3\right]+\frac{\widetilde{\mu}_{0}}{2}\left[\bar{I}_{1}-3\right]-c \mathcal{S}\left(\mathcal{I}_{5}\right)+\frac{c \varepsilon_{0_{\mathrm{p}}}}{2} \mathcal{I}_{5}+\frac{\widetilde{m}_{K_{0}}-\widetilde{\varepsilon}_{0}}{2} \bar{I}_{4}^{E}-\frac{\widetilde{m}_{K_{0}}}{2} \bar{I}_{5}^{E},
$$

where $\bar{I}_{1}=\overline{\mathbf{F}} \cdot \overline{\mathbf{F}}, \bar{I}_{4}^{E}=\overline{\mathbf{E}} \cdot \overline{\mathbf{E}}, \bar{I}_{5}^{E}=\overline{\mathbf{F}}^{-T} \overline{\mathbf{E}} \cdot \overline{\mathbf{F}}^{-T} \overline{\mathbf{E}}$ are isotropic invariants of the macroscopic deformation gradient $\overline{\mathbf{F}}$ and macroscopic Lagrangian electric field $\overline{\mathbf{E}}$,

$$
\mathcal{I}_{1}=\frac{1}{1-c} \frac{\partial \widetilde{\mu}_{0}}{\partial \mu_{0}}\left[\bar{I}_{1}-3\right]+\frac{1}{1-c} \frac{\partial \widetilde{m}_{K_{0}}}{\partial \mu_{0}}\left[\bar{I}_{4}^{E}-\bar{I}_{5}^{E}\right]+3, \quad \mathcal{I}_{5}=-\frac{1}{c}\left(\frac{\partial \widetilde{m}_{K_{0}}}{\partial \varepsilon_{0_{\mathrm{p}}}}-\frac{\partial \widetilde{\varepsilon}_{0}}{\partial \varepsilon_{0_{\mathrm{p}}}}\right) \bar{I}_{4}^{E}+\frac{1}{c} \frac{\partial \widetilde{m}_{K_{0}}}{\partial \varepsilon_{0_{\mathrm{p}}}} \bar{I}_{5}^{E},
$$

\footnotetext{
${ }^{1}$ The interested reader is referred to Lopez-Pamies et al. (2013) for a discussion of the historical development of comparison medium methods and further references on this topic.
} 
and $\widetilde{\mu}_{0}, \widetilde{\varepsilon}_{0}, \widetilde{m}_{K_{0}}$ are microstructure-dependent coefficients that are defined in terms of the solutions of two uncoupled linear pdes (given by expressions (49) below), while the variables $\mu_{0}$ and $\varepsilon_{0_{\mathrm{p}}}$ are implicitly defined as the solution of the following system of two nonlinear algebraic equations:

$$
\mathcal{F}_{1}\left\{\mu_{0}, \varepsilon_{0_{p}}\right\}=\Psi^{\prime}\left(\mathcal{I}_{1}\right)-\frac{\mu_{0}}{2}=0, \quad \mathcal{F}_{2}\left\{\mu_{0}, \varepsilon_{0_{\mathrm{p}}}\right\}=\mathcal{S}^{\prime}\left(\mathcal{I}_{5}\right)-\frac{\varepsilon_{0_{\mathrm{p}}}}{2}=0
$$

Subsections 4.1 and 4.2 report the specialization of the proposed solution to the infinitely polydisperse and the monodisperse microstructures mentioned above. Section 5 presents the FE formulation. Section 6 provides sample results determined from the analytical approximate solution derived in Section 4 together with comparisons to FE solutions. Finally, Section 7 confronts the theoretical constitutive results derived in this work to experimental data and records some concluding remarks.

\section{The problem}

The general problem to be addressed is that of determining the macroscopic elastic dielectric response of a dielectric elastomer, filled with an isotropic distribution of particles firmly bonded across interfaces, that is subjected to arbitrarily large deformations and electric fields. The sizes of the particles are taken to be much smaller than the macroscopic size. The constitutive behaviors of the dielectric elastomer and filler particles are characterized by "total" free-energy functions $W_{\mathrm{m}}$ and $W_{\mathrm{p}}$ of the deformation gradient $\mathbf{F}$ and Lagrangian electric field $\mathbf{E}$ so that the Lagrangian pointwise (or local) constitutive relation for the dielectric elastomer composite is formally given by

$$
\mathbf{S}=\frac{\partial W}{\partial \mathbf{F}}(\mathbf{X}, \mathbf{F}, \mathbf{E}) \quad \text { and } \quad \mathbf{D}=-\frac{\partial W}{\partial \mathbf{E}}(\mathbf{X}, \mathbf{F}, \mathbf{E})
$$

with

$$
W(\mathbf{X}, \mathbf{F}, \mathbf{E})=[1-\theta(\mathbf{X})] W_{\mathrm{m}}(\mathbf{F}, \mathbf{E})+\theta(\mathbf{X}) W_{\mathbf{p}}(\mathbf{F}, \mathbf{E}) .
$$

Here, $\mathbf{S}$ and $\mathbf{D}$ denote the first Piola-Kirchhoff stress tensor and Lagrangian electric displacement field, while $\theta$ is the indicator function of the spatial regions occupied collectively by the particles, taking the value of 1 if the position vector $\mathbf{X}$ lies in a particle and zero otherwise.

The dielectric elastomer composite is considered to occupy a domain $\Omega$, with boundary $\partial \Omega$, in its undeformed stress-free polarization-free configuration and, for convenience, units of length are chosen so that $\Omega$ has unit volume. The macroscopic response of the material is defined as the relation between the averages of the first Piola-Kirchhoff stress $\mathbf{S}$ and the deformation gradient $\mathbf{F}$ over the volume $\Omega$ under affine displacement and electric potential boundary conditions: $\mathbf{x}=\overline{\mathbf{F}} \mathbf{X}$ and $\Phi=-\overline{\mathbf{E}} \cdot \mathbf{X}$ on $\partial \Omega$, where the second-order tensor $\overline{\mathbf{F}}$ and vector $\overline{\mathbf{E}}$ are prescribed quantities. In this case, it follows from the divergence theorem that $\int_{\Omega} \mathbf{F}(\mathbf{X}) \mathrm{d} \mathbf{X}=\overline{\mathbf{F}}$ and $\int_{\Omega} \mathbf{E}(\mathbf{X}) \mathrm{d} \mathbf{X}=\overline{\mathbf{E}}$, and hence the derivation of the macroscopic response reduces to finding the average stress $\overline{\mathbf{S}} \doteq \int_{\Omega} \mathbf{S}(\mathbf{X}) \mathrm{d} \mathbf{X}$ and average electric displacement $\overline{\mathbf{D}} \doteq \int_{\Omega} \mathbf{D}(\mathbf{X}) \mathrm{d} \mathbf{X}$ for given $\overline{\mathbf{F}}$ and $\overline{\mathbf{E}}$. The result reads formally as (Lopez-Pamies, 2014)

$$
\overline{\mathbf{S}}=\frac{\partial \bar{W}}{\partial \overline{\mathbf{F}}}(\overline{\mathbf{F}}, \overline{\mathbf{E}}, c) \quad \text { and } \quad \overline{\mathbf{D}}=-\frac{\partial \bar{W}}{\partial \overline{\mathbf{E}}}(\overline{\mathbf{F}}, \overline{\mathbf{E}}, c)
$$

with

$$
\bar{W}(\overline{\mathbf{F}}, \overline{\mathbf{E}}, c)=\min _{\mathbf{F} \in \mathcal{K}} \max _{\mathbf{E} \in \mathcal{E}} \int_{\Omega} W(\mathbf{X}, \mathbf{F}, \mathbf{E}) \mathrm{d} \mathbf{X} .
$$

In these last expressions, $\bar{W}$ corresponds physically to the total electroelastic free energy (per unit undeformed volume) of the composite, $c=\int_{\Omega} \theta(\mathbf{X}) \mathrm{d} \mathbf{X}$ is the initial volume fraction or concentration of particles, and $\mathcal{K}, \mathcal{E}$ denote sufficiently large sets of admissible deformation gradients $\mathbf{F}$ and curl-free electric fields E consistent with the applied affine boundary conditions. 
The $\mathbf{F}$ and $\mathbf{D}$ formulation. At several passages in the sequel, it will prove useful to treat the local and macroscopic electric displacement fields $\mathbf{D}$ and $\overline{\mathbf{D}}$ as the independent local and macroscopic electric variables instead of the electric fields $\mathbf{E}$ and $\overline{\mathbf{E}}$. Among several possibilities, this can be readily accomplished with help of suitable partial Legendre transforms. Indeed, for the case of interest here when $W(\mathbf{X}, \mathbf{F}, \mathbf{E})$ is concave in $\mathbf{E}$, the partial Legendre transform

$$
W^{*}(\mathbf{X}, \mathbf{F}, \mathbf{D})=\sup _{\mathbf{E}}\{\mathbf{D} \cdot \mathbf{E}+W(\mathbf{X}, \mathbf{F}, \mathbf{E})\},
$$

which physically corresponds to the local Helmholtz free energy, allows us to write formally the local constitutive relation for the dielectric elastomer composite in the form

$$
\mathbf{S}=\frac{\partial W^{*}}{\partial \mathbf{F}}(\mathbf{X}, \mathbf{F}, \mathbf{D}) \quad \text { and } \quad \mathbf{E}=\frac{\partial W^{*}}{\partial \mathbf{D}}(\mathbf{X}, \mathbf{F}, \mathbf{D})
$$

where now $\mathbf{D}$ plays the role of independent electric variable. Similarly, for the case when $\bar{W}(\overline{\mathbf{F}}, \overline{\mathbf{E}}, c)$ is concave in $\overline{\mathbf{E}}$, the partial Legendre transform

$$
\bar{W}^{*}(\overline{\mathbf{F}}, \overline{\mathbf{D}}, c)=\sup _{\overline{\mathbf{E}}}\{\overline{\mathbf{D}} \cdot \overline{\mathbf{E}}+\bar{W}(\overline{\mathbf{F}}, \overline{\mathbf{E}}, c)\}
$$

allows us to write formally the macroscopic constitutive relation for the dielectric elastomer composite in the form

$$
\overline{\mathbf{S}}=\frac{\partial \bar{W}^{*}}{\partial \overline{\mathbf{F}}}(\overline{\mathbf{F}}, \overline{\mathbf{D}}, c) \quad \text { and } \quad \overline{\mathbf{E}}=\frac{\partial \bar{W}^{*}}{\partial \overline{\mathbf{D}}}(\overline{\mathbf{F}}, \overline{\mathbf{D}}, c)
$$

with $\overline{\mathbf{D}}$ now as the independent macroscopic electric variable.

For later reference, we note that the effective Helmholtz free energy $\bar{W}^{*}$ defined by the partial Legendre transform (7) can be computed directly from the minimization of the local Helmholtz free energy (5):

$$
\bar{W}^{*}(\overline{\mathbf{F}}, \overline{\mathbf{D}}, c)=\min _{\mathbf{F} \in \mathcal{K}} \min _{\mathbf{D} \in \mathcal{D}} \int_{\Omega} W^{*}(\mathbf{X}, \mathbf{F}, \mathbf{D}) \mathrm{d} \mathbf{X}
$$

Here, it is recalled that $\mathcal{K}$ stands for a sufficiently large set of admissible deformation gradients $\mathbf{F}$ consistent with affine boundary conditions and, similarly, $\mathcal{D}$ denotes a sufficiently large set of divergence-free electric displacement fields $\mathbf{D}$ also consistent with affine boundary conditions.

\subsection{Non-Gaussian dielectric elastomers isotropically filled with nonlinear elastic dielectric particles}

The object of this paper is to generate solutions for the effective free energy $\bar{W}$ defined by the variational problem (4) for the practically relevant case of dielectric elastomer composites wherein the distribution of filler particles is isotropic, the elastomeric matrix is non-Gaussian, and the particles are nonlinear elastic dielectrics whose polarization may possibly saturate.

Non-Gaussian dielectric elastomers. In particular, we consider in this work elastomeric matrix materials characterized by free-energy functions of the form

$$
W_{\mathrm{m}}(\mathbf{F}, \mathbf{E})=\left\{\begin{array}{ll}
\Psi\left(I_{1}\right)-\frac{\varepsilon}{2} I_{5}^{E} & \text { if } \operatorname{det} \mathbf{F}=1 \\
+\infty & \text { otherwise }
\end{array},\right.
$$

where $I_{1}=\mathbf{F} \cdot \mathbf{F}, I_{5}^{E}=\mathbf{F}^{-T} \mathbf{E} \cdot \mathbf{F}^{-T} \mathbf{E}, \Psi$ is any non-negative function of choice (suitably well-behaved) satisfying the linearization conditions

$$
\Psi(3)=0 \quad \text { and } \quad \Psi^{\prime}(3)=\frac{\mu}{2}
$$

with $\mu$ and $\varepsilon$ denoting the initial shear modulus and the initial permittivity of the material; throughout this paper, we make use of the convention $y^{\prime}(x)=\mathrm{d} y(x) / \mathrm{d} x$ to denote the derivative of functions of a single scalar variable. We recall that basic physical considerations dictate that $\mu>0$ and $\varepsilon \geqslant \varepsilon_{0}$, where $\varepsilon_{0} \approx 8.85 \times 10^{-12} \mathrm{~F} / \mathrm{m}$ stands for the permittivity of vacuum. 
We remark that free-energy functions of the form (10) have been shown to describe reasonably well the response of a broad variety of dielectric elastomers over wide ranges of deformations and electric fields (see, e.g., Wissler, 2007; Lopez-Pamies, 2010). While analytical results will be worked out in Section 4 for arbitrary choices of the function $\Psi$, in Section 6 sample numerical results will be presented in particular for the choice

$$
\Psi\left(I_{1}\right)=\frac{3^{1-\alpha_{1}}}{2 \alpha_{1}} \mu_{1}\left[I_{1}^{\alpha_{1}}-3^{\alpha_{1}}\right]+\frac{3^{1-\alpha_{2}}}{2 \alpha_{2}} \mu_{2}\left[I_{1}^{\alpha_{2}}-3^{\alpha_{2}}\right] .
$$

In this expression, $\mu_{1}, \mu_{2}, \alpha_{1}, \alpha_{2}$ are real-valued material parameters that may be associated with the non-Gaussian statistical distribution of the underlying polymer chains. In addition to its mathematical simplicity and physical meaning of its parameters, we choose this class of functions because of its rich functional form and demonstrated descriptive and predictive capabilities (Lopez-Pamies, 2010).

For later use, we note that the partial Legendre transform (5) corresponding to the local free-energy function (10) is simply given by

$$
W_{\mathrm{m}}^{*}(\mathbf{F}, \mathbf{D})=\sup _{\mathbf{E}}\left\{\mathbf{D} \cdot \mathbf{E}+W_{\mathrm{m}}(\mathbf{F}, \mathbf{E})\right\}=\left\{\begin{array}{ll}
\Psi\left(I_{1}\right)+\frac{1}{2 \varepsilon} I_{5}^{D} & \text { if } \operatorname{det} \mathbf{F}=1 \\
+\infty & \text { otherwise }
\end{array},\right.
$$

where $I_{5}^{D}=\mathbf{F D} \cdot \mathbf{F D}$.

Nonlinear elastic dielectric particles. On the other hand, we consider the elastic dielectric behavior of the filler particles to be characterized by free-energy functions of the form

$$
W_{\mathbf{p}}(\mathbf{F}, \mathbf{E})=\left\{\begin{array}{ll}
\frac{\mu_{\mathrm{p}}}{2}\left[I_{1}-3\right]-\mathcal{S}\left(I_{5}^{E}\right) & \text { if } \operatorname{det} \mathbf{F}=1 \\
+\infty & \text { otherwise }
\end{array},\right.
$$

where, again, $I_{1}=\mathbf{F} \cdot \mathbf{F}, I_{5}^{E}=\mathbf{F}^{-T} \mathbf{E} \cdot \mathbf{F}^{-T} \mathbf{E}, \mu_{\mathrm{p}}$ stands for the initial shear modulus of the particles, and $\mathcal{S}$ is any function of choice satisfying the linearization conditions

$$
\mathcal{S}(0)=0 \quad \text { and } \quad \mathcal{S}^{\prime}(0)=\frac{\varepsilon_{\mathrm{p}}}{2}
$$

with $\varepsilon_{\mathrm{p}}$ denoting the initial permittivity of the particles, and the convexity conditions

$$
\mathcal{S}^{\prime}\left(I_{5}^{E}\right)>0 \text { and } \mathcal{S}^{\prime}\left(I_{5}^{E}\right)+2 I_{5}^{E} \mathcal{S}^{\prime \prime}\left(I_{5}^{E}\right)>0 .
$$

The inequalities (16) on the function $\mathcal{S}$ ensure that the free energy (14) is, much like (10), concave in E.

Free-energy functions of the form (14) are general enough to adequately model the elastic dielectric responses over wide ranges of deformations and electric fields of hard polymers such as polyaniline (PANI) and copper phthalocyanine oligomers $(\mathrm{O}-\mathrm{CuPc})$, ceramics such as titania $\left(\mathrm{TiO}_{2}\right)$, and liquids such as Galistan, which have been utilized/suggested as filler particles in experimental investigations (see, e.g., Li et al., 2004; Huang et al., 2005; Liu et al., 2013; Fassler and Majidi, 2015). We emphasize in particular that free-energy functions of the form (14) are general enough to model (albeit ignoring dissipative effects) polarization saturation phenomena typical, for instance, of ferroelectric ceramics at large electric fields. In this case, noting that the polarization $\mathbf{p}$ (per unit deformed volume) implied by (14) is given by the expression

$$
\mathbf{p}=-\mathbf{F} \frac{\partial W_{\mathrm{p}}}{\partial \mathbf{E}}(\mathbf{F}, \mathbf{E})-\varepsilon_{0} \mathbf{F}^{-T} \mathbf{E}=\left[2 \mathcal{S}^{\prime}\left(I_{5}^{E}\right)-\varepsilon_{0}\right] \mathbf{F}^{-T} \mathbf{E}
$$

in addition to the linearization and convexity conditions (15)-(16), it must be required that

$$
\mathcal{S}^{\prime}\left(I_{5}^{E}\right)=\frac{\varepsilon_{0}}{2}+\frac{p_{s}}{2 \sqrt{I_{5}^{E}}}+o\left(1 / \sqrt{I_{5}^{E}}\right)
$$

in the limit as $I_{5}^{E} \rightarrow \infty$. In this last expression, the positive material constant $p_{s}$ characterizes the magnitude of the saturated polarization. While analytical results will be worked out in Section 4 for any 
function $\mathcal{S}$ of choice, in Section 6 sample numerical results will be presented in particular for the classical polarization-saturation model due to Debye (Langevin, 1905; Debye, 1929):

$$
\mathcal{S}\left(I_{5}^{E}\right)=\frac{\varepsilon_{0}}{2} I_{5}^{E}+\frac{p_{s}^{2}}{3\left(\varepsilon_{\mathrm{p}}-\varepsilon_{0}\right)}\left[\ln \left(\sinh \left(\frac{3\left(\varepsilon_{\mathrm{p}}-\varepsilon_{0}\right) \sqrt{I_{5}^{E}}}{p_{s}}\right)\right)-\ln \left(\frac{3\left(\varepsilon_{\mathrm{p}}-\varepsilon_{0}\right) \sqrt{I_{5}^{E}}}{p_{s}}\right)\right] .
$$

Also for later use, we note that the partial Legendre transform (5) corresponding to the local free-energy function (14) is given by

$$
W_{\mathrm{p}}^{*}(\mathbf{F}, \mathbf{D})=\sup _{\mathbf{E}}\left\{\mathbf{D} \cdot \mathbf{E}+W_{\mathrm{p}}(\mathbf{F}, \mathbf{E})\right\}=\left\{\begin{array}{ll}
\frac{\mu_{\mathrm{p}}}{2}\left[I_{1}-3\right]+\mathcal{S}^{*}\left(I_{5}^{D}\right) & \text { if } \operatorname{det} \mathbf{F}=1 \\
+\infty & \text { otherwise }
\end{array},\right.
$$

where, again, $I_{5}^{D}=\mathbf{F D} \cdot \mathbf{F D}$ and $\mathcal{S}^{*}\left(I_{5}^{D}\right)=I_{5}^{D} \mathcal{S}^{\prime}\left(z^{-1}\left(I_{5}^{D}\right)\right) / 2-\mathcal{S}\left(z^{-1}\left(I_{5}^{D}\right)\right)$ with $z^{-1}$ denoting the inverse of the function $z$ defined by $z(x)=4 x\left(\mathcal{S}^{\prime}(x)\right)^{2}$. Given the properties (15)-(16) of the function $\mathcal{S}$, it follows that

and

$$
\mathcal{S}^{*}(0)=0, \quad \mathcal{S}^{* \prime}(0)=\frac{1}{2 \varepsilon_{\mathrm{p}}},
$$

$$
\mathcal{S}^{* \prime}\left(I_{5}^{D}\right)>0, \quad \mathcal{S}^{* \prime}\left(I_{5}^{D}\right)+2 I_{5}^{D} \mathcal{S}^{* \prime \prime}\left(I_{5}^{D}\right)>0
$$

the latter of which implies that the free-energy function (20) is convex in $\mathbf{D}$, in agreement with its very definition.

The macroscopic response. Owing to the assumed isotropy of the microstructure and the constitutive isotropy and incompressibility of the matrix material (10) and filler particles (14), the resulting macroscopic elastic dielectric response is isotropic and incompressible. This implies that the effective free-energy function (4) in this case only depends on the macroscopic deformation gradient $\overline{\mathbf{F}}$ and macroscopic Lagrangian electric field $\overline{\mathbf{E}}$ through five invariants and becomes unbounded for non-isochoric deformations when $\operatorname{det} \overline{\mathbf{F}} \neq 1$. Consistent with the variables employed in Part I, with a slight abuse of notation, we write

$$
\bar{W}(\overline{\mathbf{F}}, \overline{\mathbf{E}}, c)= \begin{cases}\bar{W}\left(\bar{I}_{1}, \bar{I}_{2}, \bar{I}_{4}^{E}, \bar{I}_{5}^{E}, \bar{I}_{6}^{E}, c\right) & \text { if } \operatorname{det} \overline{\mathbf{F}}=1 \\ +\infty & \text { otherwise }\end{cases}
$$

in terms of the five standard invariants

$$
\bar{I}_{1}=\overline{\mathbf{F}} \cdot \overline{\mathbf{F}}, \quad \bar{I}_{2}=\overline{\mathbf{F}}^{-T} \cdot \overline{\mathbf{F}}^{-T}, \quad \bar{I}_{4}^{E}=\overline{\mathbf{E}} \cdot \overline{\mathbf{E}}, \quad \bar{I}_{5}^{E}=\overline{\mathbf{F}}^{-T} \overline{\mathbf{E}} \cdot \overline{\mathbf{F}}^{-T} \overline{\mathbf{E}}, \quad \bar{I}_{6}^{E}=\overline{\mathbf{F}}^{-1} \overline{\mathbf{F}}^{-T} \overline{\mathbf{E}} \cdot \overline{\mathbf{F}}^{-1} \overline{\mathbf{F}}^{-T} \overline{\mathbf{E}} .
$$

Similarly, we write the partial Legendre transform (7) of the effective free-energy function (23) as

$$
\bar{W}^{*}(\overline{\mathbf{F}}, \overline{\mathbf{D}}, c)= \begin{cases}\bar{W}^{*}\left(\bar{I}_{1}, \bar{I}_{2}, \bar{I}_{4}^{D}, \bar{I}_{5}^{D}, \bar{I}_{6}^{D}, c\right) & \text { if } \operatorname{det} \overline{\mathbf{F}}=1 \\ +\infty & \text { otherwise }\end{cases}
$$

in terms of the five standard invariants $(24)_{1,2}$ and

$$
\bar{I}_{4}^{D}=\overline{\mathbf{D}} \cdot \overline{\mathbf{D}}, \quad \bar{I}_{5}^{D}=\overline{\mathbf{F}} \overline{\mathbf{D}} \cdot \overline{\mathbf{F}} \overline{\mathbf{D}}, \quad \bar{I}_{6}^{D}=\overline{\mathbf{F}}^{T} \overline{\mathbf{F}} \overline{\mathbf{D}} \cdot \overline{\mathbf{F}}^{T} \overline{\mathbf{F}} \overline{\mathbf{D}} .
$$

\section{A nonlinear comparison medium method in nonlinear electroelastostatics}

In this section, we put forth a comparison medium method that allows us to generate variational approximations for the effective free-energy function $\bar{W}$ of a given two-phase elastic dielectric composite, as defined by the variational problem (4), in terms of the effective free-energy function, $\bar{W}_{0}$ say, of another elastic dielectric composite (possibly comprising finitely or infinitely many phases). For clarity of exposition, the method is presented in its general form. Its specialization to the non-Gaussian dielectric 
elastomers isotropically filled with nonlinear elastic dielectric particles of interest in this work will be presented in Section 4.

As will become apparent below, contrary to the minimax nature of the variational problem (4) defining $\bar{W}$, the overall minimum nature of the variational problem (9) defining $\bar{W}^{*}$ shall prove gainful in our strategy. We thus begin by considering an elastic dielectric composite characterized locally by the (possibly compressible and anisotropic) two-phase Helmholtz free-energy function $W^{*}(\mathbf{X}, \mathbf{F}, \mathbf{D})=$ $[1-\theta(\mathbf{X})] W_{\mathrm{m}}^{*}(\mathbf{F}, \mathbf{D})+\theta(\mathbf{X}) W_{\mathrm{p}}^{*}(\mathbf{F}, \mathbf{D})$, where the indicator function $\theta$ is the same as in (2). Following Lopez-Pamies et al. (2013), we consider as well an arbitrary comparison medium with pointwise free energy $W_{0}^{*}(\mathbf{X}, \mathbf{F}, \mathbf{D})$, introduce the functions $f=f(\mathbf{X}, \mathbf{F}, J, \mathbf{D})$ and $f_{0}=f_{0}(\mathbf{X}, \mathbf{F}, J, \mathbf{D})$ such that

$$
f(\mathbf{X}, \mathbf{F}, J, \mathbf{D})=W^{*}(\mathbf{X}, \mathbf{F}, \mathbf{D})=[1-\theta(\mathbf{X})] W_{\mathrm{m}}^{*}(\mathbf{F}, \mathbf{D})+\theta(\mathbf{X}) W_{\mathrm{p}}^{*}(\mathbf{F}, \mathbf{D}) \quad \text { when } \quad J=\operatorname{det} \mathbf{F}
$$

and

$$
f_{0}(\mathbf{X}, \mathbf{F}, J, \mathbf{D})=W_{0}^{*}(\mathbf{X}, \mathbf{F}, \mathbf{D}) \text { when } J=\operatorname{det} \mathbf{F},
$$

and define the Legendre transform

$$
\left(f-f_{0}\right)^{*}(\mathbf{X}, \mathbf{P}, Q, \mathbf{R}) \doteq \sup _{\mathbf{F}, J, \mathbf{D}}\left[\mathbf{F} \cdot \mathbf{P}+J Q+\mathbf{D} \cdot \mathbf{R}-f(\mathbf{X}, \mathbf{F}, J, \mathbf{D})+f_{0}(\mathbf{X}, \mathbf{F}, J, \mathbf{D})\right] .
$$

Now, for any $\mathbf{P}, Q$, and $\mathbf{R}$ it follows from (29) that

$$
f(\mathbf{X}, \mathbf{F}, J, \mathbf{D}) \geq f_{0}(\mathbf{X}, \mathbf{F}, J, \mathbf{D})+\mathbf{F} \cdot \mathbf{P}+J Q+\mathbf{D} \cdot \mathbf{R}-\left(f-f_{0}\right)^{*}(\mathbf{X}, \mathbf{P}, Q, \mathbf{R})
$$

and therefore that

$$
\bar{W}^{*}(\overline{\mathbf{F}}, \overline{\mathbf{D}}, c) \geq \min _{\mathbf{F} \in \mathcal{K}} \min _{\mathbf{D} \in \mathcal{D}} \int_{\Omega}\left[f_{0}(\mathbf{X}, \mathbf{F}, J, \mathbf{D})+\mathbf{F} \cdot \mathbf{P}+J Q+\mathbf{D} \cdot \mathbf{R}\right] \mathrm{d} \mathbf{X}-\int_{\Omega}\left(f-f_{0}\right)^{*}(\mathbf{X}, \mathbf{P}, Q, \mathbf{R}) \mathrm{d} \mathbf{X}
$$

where $J=\operatorname{det} \mathbf{F}$ here and henceforth. We recall that the minima sought in the right-hand side of (31) are over the sets $\mathcal{K}$ and $\mathcal{D}$ of admissible deformation gradients $\mathbf{F}(\mathbf{X})$ with prescribed volume average $\overline{\mathbf{F}}$ and of admissible divergence-free electric displacement fields $\mathbf{D}(\mathbf{X})$ with volume average $\overline{\mathbf{D}}$. A well-known property of minima of sums yields the further inequality

$$
\begin{aligned}
\bar{W}^{*}(\overline{\mathbf{F}}, \overline{\mathbf{D}}, c) \geq & \bar{W}_{0}^{*}(\overline{\mathbf{F}}, \overline{\mathbf{D}})+\min _{\mathbf{F} \in \mathcal{K}} \int_{\Omega} \mathbf{F} \cdot \mathbf{P} \mathrm{d} \mathbf{X}+\min _{\mathbf{F} \in \mathcal{K}} \int_{\Omega} J Q \mathrm{~d} \mathbf{X}+\min _{\mathbf{D} \in \mathcal{D}} \int_{\Omega} \mathbf{D} \cdot \mathbf{R} \mathrm{d} \mathbf{X}- \\
& \int_{\Omega}\left(f-f_{0}\right)^{*}(\mathbf{X}, \mathbf{P}, Q, \mathbf{R}) \mathrm{d} \mathbf{X},
\end{aligned}
$$

where the notation $\bar{W}_{0}^{*}$ has been introduced to denote the effective free-energy function of the comparison medium with local free energy $f_{0}(\mathbf{X}, \mathbf{F}, J, \mathbf{D})=W_{0}^{*}(\mathbf{X}, \mathbf{F}, \mathbf{D})$ :

$$
\bar{W}_{0}^{*}(\mathbf{F}, \mathbf{D})=\min _{\mathbf{F} \in \mathcal{K}} \min _{\mathbf{D} \in \mathcal{D}} \int_{\Omega} f_{0}(\mathbf{X}, \mathbf{F}, J, \mathbf{D}) \mathrm{d} \mathbf{X} .
$$

The second, third, and fourth terms in the right-hand side of (32) are bounded from below provided that $\mathbf{P}$ is a divergence-free field, $Q$ is a constant, and $\mathbf{R}$ is a curl-free field. For simplicity, we select all these fields to be constant and denote them by $\mathbf{P}=\overline{\mathbf{P}}, Q=\bar{Q}$, and $\mathbf{R}=\overline{\mathbf{R}}$. After a standard calculation, this simplifying prescription gives

$$
\bar{W}^{*}(\overline{\mathbf{F}}, \overline{\mathbf{D}}, c) \geq \bar{W}_{0}^{*}(\overline{\mathbf{F}}, \overline{\mathbf{D}})+\overline{\mathbf{F}} \cdot \overline{\mathbf{P}}+\bar{J} \bar{Q}+\overline{\mathbf{D}} \cdot \overline{\mathbf{R}}-\int_{\Omega}\left(f-f_{0}\right)^{*}(\mathbf{X}, \overline{\mathbf{P}}, \bar{Q}, \overline{\mathbf{R}}) \mathrm{d} \mathbf{X}
$$

where the definition $\bar{J} \doteq \operatorname{det} \overline{\mathbf{F}}$ has been employed.

The inequality (34) is valid for any choice of constant fields $\overline{\mathbf{P}}, \bar{Q}$, and $\overline{\mathbf{R}}$, as well as for any choice of local free-energy function $f_{0}(\mathbf{X}, \mathbf{F}, J, \mathbf{D})$ describing the microstructure and local elastic dielectric behavior 
of the comparison medium. Optimizing with respect to $\overline{\mathbf{P}}, \bar{Q}$, and $\overline{\mathbf{R}}$ yields

$$
\begin{aligned}
\bar{W}^{*}(\overline{\mathbf{F}}, \overline{\mathbf{D}}, c) & \geq \bar{W}_{0}^{*}(\overline{\mathbf{F}}, \overline{\mathbf{D}})+\sup _{\overline{\mathbf{P}}, \bar{Q}, \overline{\mathbf{R}}}\left\{\overline{\mathbf{F}} \cdot \overline{\mathbf{P}}+\bar{J} \bar{Q}+\overline{\mathbf{D}} \cdot \overline{\mathbf{R}}-\int_{\Omega}\left(f-f_{0}\right)^{*}(\mathbf{X}, \overline{\mathbf{P}}, \bar{Q}, \overline{\mathbf{R}}) \mathrm{d} \mathbf{X}\right\} \\
& \geq \bar{W}_{0}^{*}(\overline{\mathbf{F}}, \overline{\mathbf{D}})+\left(\int_{\Omega}\left(f-f_{0}\right)^{*} \mathrm{~d} \mathbf{X}\right)^{*}(\overline{\mathbf{F}}, \bar{J}, \overline{\mathbf{D}}) .
\end{aligned}
$$

Optimizing in turn with respect to $f_{0}$ leads formally to

$$
\bar{W}^{*}(\overline{\mathbf{F}}, \overline{\mathbf{D}}, c) \geq \sup _{f_{0}}\left\{\bar{W}_{0}^{*}(\overline{\mathbf{F}}, \overline{\mathbf{D}})+\left(\int_{\Omega}\left(f-f_{0}\right)^{*} \mathrm{~d} \mathbf{X}\right)^{*}(\overline{\mathbf{F}}, \bar{J}, \overline{\mathbf{D}})\right\} .
$$

\subsection{A partially optimized explicit formulation}

The computation of the optimized bound (36) involves two technical difficulties. The first one is that the function $\left(f-f_{0}\right)^{*}$ may exhibit corners, and hence the computation of the Legendre transform of its average in (36) may require the use of subgradients as opposed to standard differentiation; this is a difficulty that already appears in the classical context of convex energies of a single field (see, e.g., Willis, 1991). The second and more severe technical obstacle is that the supremum operation in (36) involves the optimization with respect to the microstructure of the comparison medium (in addition, as well, to the optimization with respect to its constitutive elastic dielectric behavior). Such an optimization requires the computation of complicated integrals involving the product of different indicator functions - the indicator function $\theta(\mathbf{X})$ associated with $f$ and the indicator function, $\theta_{0}(\mathbf{X})$ say, associated with $f_{0}$ in the second term of the right-hand side of (36). In this work, in the interest of analytical tractability, we shall be content with employing a partially optimized version of the result (34) - and not the fully optimized bound (36) - which avoids the two above-mentioned technical difficulties altogether.

Thus, in order to avoid the use of subgradients we set $\overline{\mathbf{P}}=\mathbf{0}, \bar{Q}=0$, and $\overline{\mathbf{R}}=\mathbf{0}$ so that, upon recognizing the string of equalities

$$
\left(f-f_{0}\right)^{*}(\mathbf{X}, \mathbf{0}, 0, \mathbf{0})=\sup _{\mathbf{A}, a, \mathbf{B}}\left[-f(\mathbf{X}, \mathbf{A}, a, \mathbf{B})+f_{0}(\mathbf{X}, \mathbf{A}, a, \mathbf{B})\right]=-\inf _{\mathbf{A}, a, \mathbf{B}}\left[f(\mathbf{X}, \mathbf{A}, a, \mathbf{B})-f_{0}(\mathbf{X}, \mathbf{A}, a, \mathbf{B})\right],
$$

relation (34) reduces to

$$
\bar{W}^{*}(\overline{\mathbf{F}}, \overline{\mathbf{D}}, c) \geq \bar{W}_{0}^{*}(\overline{\mathbf{F}}, \overline{\mathbf{D}})+\int_{\Omega} \inf _{\mathbf{A}, a, \mathbf{B}}\left[f(\mathbf{X}, \mathbf{A}, a, \mathbf{B})-f_{0}(\mathbf{X}, \mathbf{A}, a, \mathbf{B})\right] \mathrm{d} \mathbf{X} .
$$

Further, in order to avoid the computation of complicated integrals in (38) we restrict attention to comparison media with the same microstructure as the actual elastic dielectric composite, namely,

$$
f_{0}(\mathbf{X}, \mathbf{F}, J, \mathbf{D})=[1-\theta(\mathbf{X})] f_{0_{\mathrm{m}}}(\mathbf{F}, J, \mathbf{D})+\theta(\mathbf{X}) f_{0_{\mathrm{p}}}(\mathbf{F}, J, \mathbf{D}),
$$

where the indicator function $\theta$ is the same as in (2). With this choice, relation (38) leads ${ }^{2}$ to the following lower bound for $\bar{W}^{*}$ :

$$
\bar{W}^{*}(\overline{\mathbf{F}}, \overline{\mathbf{D}}, c) \geq \bar{W}_{0}^{*}(\overline{\mathbf{F}}, \overline{\mathbf{D}})+(1-c) \inf _{\mathbf{A}, a, \mathbf{B}}\left[f_{\mathrm{m}}(\mathbf{A}, a, \mathbf{B})-f_{0_{\mathrm{m}}}(\mathbf{A}, a, \mathbf{B})\right]+c \inf _{\mathbf{A}, a, \mathbf{B}}\left[f_{\mathrm{p}}(\mathbf{A}, a, \mathbf{B})-f_{0_{\mathrm{p}}}(\mathbf{A}, a, \mathbf{B})\right],
$$

where, in analogy with (27), we have made use of the notation $f_{\mathrm{m}}(\mathbf{F}, J, \mathbf{D})=W_{\mathrm{m}}^{*}(\mathbf{F}, \mathbf{D})$ and $f_{\mathrm{p}}(\mathbf{F}, J, \mathbf{D})=$ $W_{\mathrm{p}}^{*}(\mathbf{F}, \mathbf{D})$. Moreover, the symmetry of relation $(38)$ in the pairs $\left(\bar{W}^{*}, f\right)$ and $\left(\bar{W}_{0}^{*}, f_{0}\right)$ implies as well the following upper bound:

$$
\bar{W}^{*}(\overline{\mathbf{F}}, \overline{\mathbf{D}}, c) \leq \bar{W}_{0}^{*}(\overline{\mathbf{F}}, \overline{\mathbf{D}})+(1-c) \sup _{\mathbf{A}, a, \mathbf{B}}\left[f_{\mathrm{m}}(\mathbf{A}, a, \mathbf{B})-f_{0_{\mathrm{m}}}(\mathbf{A}, a, \mathbf{B})\right]+c \sup _{\mathbf{A}, a, \mathbf{B}}\left[f_{\mathrm{p}}(\mathbf{A}, a, \mathbf{B})-f_{0_{\mathrm{p}}}(\mathbf{A}, a, \mathbf{B})\right] .
$$

\footnotetext{
${ }^{2}$ An alternative direct derivation of the formula (40) follows from a straightforward extension of the derivation of Willis (see, e.g., equation (3.3) in Willis, 1991; see also deBotton and Shmuel, 2010) of Ponte Castañeda's bound (1991) in the context of convex energies of a single field: $\bar{W}^{*}=\min _{\mathbf{F} \in \mathcal{K}} \min _{\mathbf{D} \in \mathcal{D}} \int_{\Omega}\left[W_{0}^{*}+\left(W^{*}-W_{0}^{*}\right)\right] \mathrm{d} \mathbf{X} \geq \bar{W}_{0}+\int_{\Omega} \min \left(W^{*}-W_{0}^{*}\right) \mathrm{d} \mathbf{X}$. 
Note that the lower bound (40) is non-trivial provided that $f_{\mathrm{m}}-f_{0_{\mathrm{m}}}>-\infty$ and $f_{\mathrm{p}}-f_{0_{\mathrm{p}}}>-\infty$, while the upper bound (41) is non-trivial provided that $f_{\mathrm{m}}-f_{0_{\mathrm{m}}}<\infty$ and $f_{\mathrm{p}}-f_{0_{\mathrm{p}}}<\infty$. Combining the inequalities (40) and (41) leads to the following approximate solution for the effective free-energy function (9):

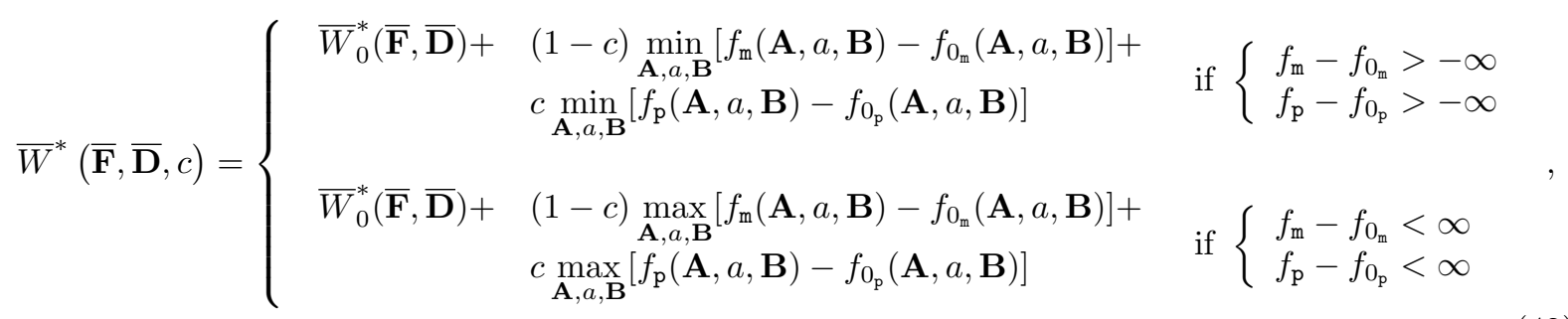

where the equality in this last expression has been used in the sense of a variational approximation. The result (42) is valid for any choice of free-energy functions $f_{0_{\mathrm{m}}}$ and $f_{0_{\mathrm{p}}}$ describing the elastic dielectric behaviors of the matrix and filler particles in the comparison medium. Optimizing (42) with respect to these free energies leads formally to

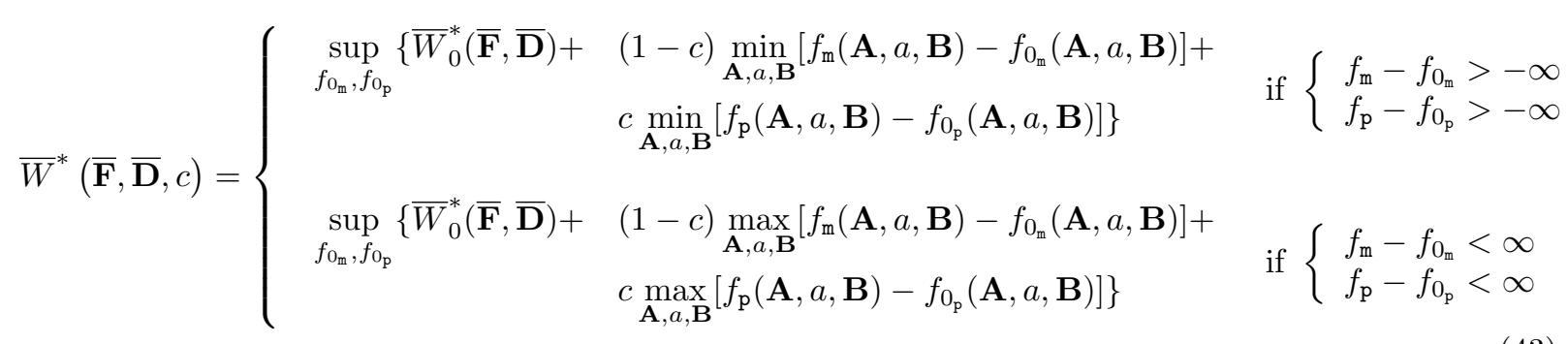

Expression (43) constitutes the main result of this section: it provides a variational approximation for the effective free-energy function (9) of a two-phase elastic dielectric composite with local free energy (27) in terms of the effective free-energy function (33) of another two-phase elastic dielectric composite with local free energy (39). For the case when the effective Helmholtz free-energy function $\bar{W}^{*}$ resulting from (43) is convex in $\overline{\mathbf{D}}$, the corresponding effective free-energy function $\bar{W}$ in terms of $\overline{\mathbf{F}}$ and $\overline{\mathbf{E}}$ can be expediently computed via the following partial Legendre transform:

$$
\bar{W}(\overline{\mathbf{F}}, \overline{\mathbf{E}}, c)=-\sup _{\overline{\mathbf{D}}}\left\{\overline{\mathbf{D}} \cdot \overline{\mathbf{E}}-\bar{W}^{*}(\overline{\mathbf{F}}, \overline{\mathbf{D}}, c)\right\} .
$$

It is plain that the usefulness of the variational approximation (43) - or more generally (42) - hinges upon having knowledge of the effective free-energy function $\bar{W}_{0}^{*}$ for the comparison medium. While there have been no prior results available heretofore, we now have at our disposal the results for ideal elastic dielectric composites worked out in Part I.

\section{Application to non-Gaussian dielectric elastomers isotropically filled with nonlinear elas- tic dielectric particles}

In the sequel, we make use of the ideal elastic dielectric composites considered in Part I as choices for the comparison medium in the formulation (43)-(44) in order to construct an approximate homogenization solution for the effective free energy (4) of non-Gaussian dielectric elastomers, characterized by free-energy functions of the form (10), isotropically filled with nonlinear elastic dielectric particles characterized by free-energy functions of the form (14).

We begin by setting

$$
f_{\mathrm{m}}(\mathbf{F}, J, \mathbf{D})=\left\{\begin{array}{ll}
\Psi\left(I_{1}\right)+\frac{1}{2 \varepsilon} I_{5}^{D} & \text { if } J=1 \\
+\infty & \text { otherwise }
\end{array}, \quad f_{\mathrm{p}}(\mathbf{F}, J, \mathbf{D})=\left\{\begin{array}{ll}
\frac{\mu_{\mathrm{p}}}{2}\left[I_{1}-3\right]+\mathcal{S}^{*}\left(I_{5}^{D}\right) & \text { if } J=1 \\
+\infty & \text { otherwise }
\end{array},\right.\right.
$$


and

$f_{0_{\mathrm{m}}}(\mathbf{F}, J, \mathbf{D})=\left\{\begin{array}{ll}\frac{\mu_{0}}{2}\left[I_{1}-3\right]+\frac{1}{2 \varepsilon_{0_{\mathrm{m}}}} I_{5}^{D} & \text { if } J=1 \\ +\infty & \text { otherwise }\end{array} \quad, \quad f_{0_{\mathrm{p}}}(\mathbf{F}, J, \mathbf{D})=\left\{\begin{array}{ll}\frac{\mu_{0_{\mathrm{p}}}}{2}\left[I_{1}-3\right]+\frac{1}{2 \varepsilon_{0_{\mathrm{p}}}} I_{5}^{D} & \text { if } J=1 \\ +\infty & \text { otherwise }\end{array}\right.\right.$.

Upon substitution of these expressions in the general variational approximation (43), it is not difficult to deduce that the optimal comparison medium is such that $\varepsilon_{0_{\mathrm{m}}}=\varepsilon, \mu_{0_{\mathrm{p}}}=\mu_{\mathrm{p}}$ and, moreover, that

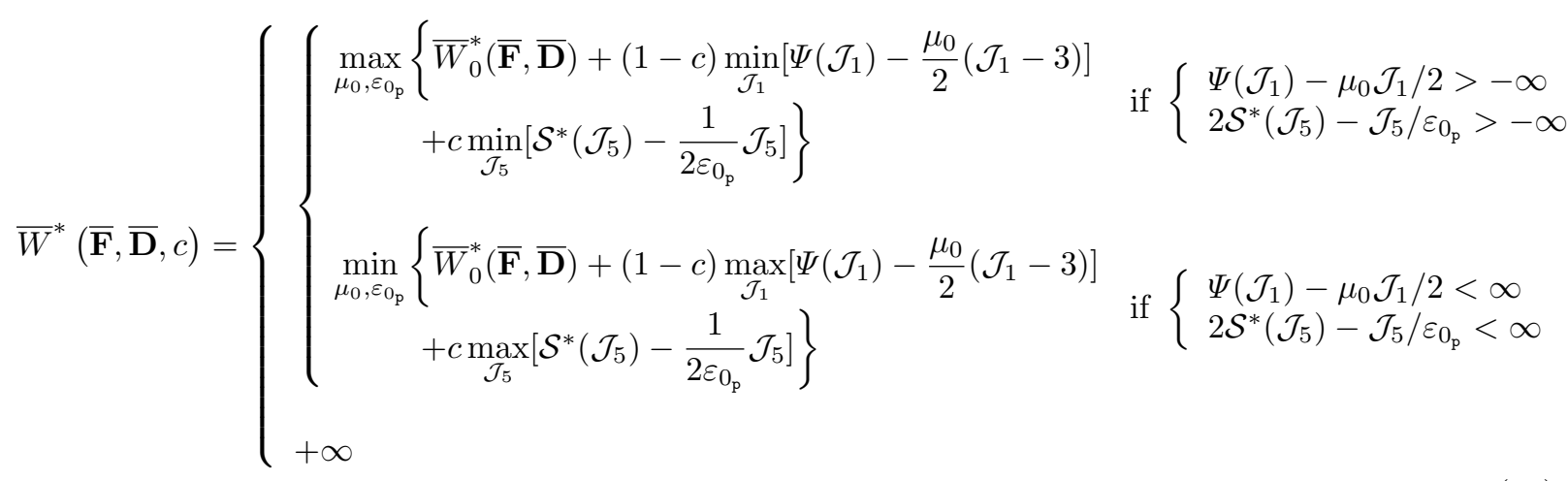

In this result, it is worth remarking that the macroscopic incompressibility constraint $\bar{J}=1$ that ensues from the local incompressibility constraint $J=1$ in (45)-(46) is indeed the exact constraint (within the broad context of comparison medium methods, this is a highly non-trivial result, see, e.g., Lahellec et al. (2004); deBotton and Shmuel (2010)).

Next, we recall from Part I that the effective free-energy function (33) of an elastic dielectric composite wherein the matrix and filler particles are characterized by the ideal elastic dielectric free energies (46) reads as

$$
\bar{W}_{0}^{*}(\overline{\mathbf{F}}, \overline{\mathbf{D}})=\left\{\begin{array}{ll}
\frac{\widetilde{\mu}_{0}}{2}\left[\bar{I}_{1}-3\right]+\frac{1}{2 \widetilde{m}_{K_{0}}}\left[\frac{\bar{I}_{5}^{D}+\widetilde{\eta}_{0}^{2} \bar{I}_{4}^{D}+\widetilde{\eta}_{0}\left[\bar{I}_{1} \bar{I}_{5}^{D}-\bar{I}_{6}^{D}\right]}{1+\widetilde{\eta}_{0}^{3}+\widetilde{\eta}_{0}^{2} \bar{I}_{2}+\widetilde{\eta}_{0} \bar{I}_{1}}\right] & \text { if } \bar{J}=1 \\
+\infty & \text { otherwise }
\end{array},\right.
$$

where the coefficient $\widetilde{\eta}_{0}=\left(\widetilde{\varepsilon}_{0}-\widetilde{m}_{K_{0}}\right) / \widetilde{m}_{K_{0}}$ has been introduced to ease notation and the effective coefficients $\widetilde{\mu}_{0}, \widetilde{\varepsilon}_{0}, \widetilde{m}_{K_{0}}$ are given by

$$
\begin{aligned}
& \widetilde{\mu}_{0}=\frac{1}{5} \int_{\Omega}\left([1-\theta(\mathbf{X})] \mu_{0}+\theta(\mathbf{X}) \mu_{\mathrm{p}}\right) \mathcal{K}_{k l m n} \Gamma_{m k l, n} \mathrm{~d} \mathbf{X}, \\
& \widetilde{\varepsilon}_{0}=\int_{\Omega}\left([1-\theta(\mathbf{X})] \varepsilon+\theta(\mathbf{X}) \varepsilon_{0_{\mathrm{p}}}\right) \gamma_{m, m} \mathrm{~d} \mathbf{X}, \\
& \widetilde{m}_{K_{0}}=\frac{1}{5} \int_{\Omega}\left([1-\theta(\mathbf{X})] \varepsilon+\theta(\mathbf{X}) \varepsilon_{0_{\mathrm{p}}}\right) \mathcal{K}_{i j k l} \Gamma_{r i j, s} \mathcal{K}_{r s u v} \gamma_{u, k} \gamma_{v, l} \mathrm{~d} \mathbf{X} .
\end{aligned}
$$

In these formulae, explicit use has been made of the optimality conditions $\varepsilon_{0_{\mathrm{m}}}=\varepsilon$ and $\mu_{0_{\mathrm{p}}}=\mu_{\mathrm{p}}, \mathcal{K}_{i j k l}=$ $1 / 2\left(\delta_{i k} \delta_{j l}+\delta_{i l} \delta_{j k}\right)-1 / 3 \delta_{i j} \delta_{k l}$ with $\delta_{i j}$ denoting the Kronecker delta, the notation ${ }_{i}$ represents partial differentiation with respect to the material point coordinate $X_{i}$, and the tensor fields $\boldsymbol{\Gamma}$ and $\gamma$ are defined as the solutions of the following uncoupled linear boundary value problems:

$$
\left\{\begin{array}{l}
{\left[\left([1-\theta(\mathbf{X})] \mu_{0}+\theta(\mathbf{X}) \mu_{\mathrm{p}}\right) \mathcal{K}_{i j m n} \Gamma_{m k l, n}\right]_{, j}=0} \\
\Gamma_{m k l, m}=0
\end{array} \text { for } \mathbf{X} \in \Omega, \quad \Gamma_{i k l}=\delta_{i k} X_{l} \text { for } \mathbf{X} \in \partial \Omega\right.
$$

and

$$
\left[\left([1-\theta(\mathbf{X})] \varepsilon+\theta(\mathbf{X}) \varepsilon_{0_{\mathrm{p}}}\right) \gamma_{i, j}\right]_{, i}=0 \quad \text { for } \mathbf{X} \in \Omega, \quad \gamma_{i}=X_{i} \text { for } \mathbf{X} \in \partial \Omega
$$

if $\bar{J}=1$

otherwise 
Granted the result (48) for the effective free-energy function of the comparison medium, irrespectively of the growth conditions of the functions $\Psi$ and $\mathcal{S}^{*}$, the variational approximation (47) can be shown to reduce to

$$
\bar{W}^{*}(\overline{\mathbf{F}}, \overline{\mathbf{D}}, c)= \begin{cases}(1-c) \Psi\left(\mathcal{J}_{1}\right)-\frac{\mu_{0}(1-c)}{2}\left[\mathcal{J}_{1}-3\right]+\frac{\widetilde{\mu}_{0}}{2}\left[\bar{I}_{1}-3\right]+ & \\ c \mathcal{S}^{*}\left(\mathcal{J}_{5}\right)-\frac{c}{2 \varepsilon_{0_{\mathrm{p}}}} \mathcal{J}_{5}+\frac{1}{2 \widetilde{m}_{K_{0}}}\left[\frac{\bar{I}_{5}^{D}+\widetilde{\eta}_{0}^{2} \bar{I}_{4}^{D}+\widetilde{\eta}_{0}\left[\bar{I}_{1} \bar{I}_{5}^{D}-\bar{I}_{6}^{D}\right]}{\left.1+\widetilde{\eta}_{0}^{3}+\widetilde{\eta}_{0}^{2} \bar{I}_{2}+\widetilde{\eta}_{0} \bar{I}_{1}\right]}\right. & \text { if } \bar{J}=1 \\ +\infty & \text { otherwise }\end{cases}
$$

with

$$
\begin{aligned}
\mathcal{J}_{1}= & \frac{1}{1-c}\left[\left(\bar{I}_{1}-3\right) \frac{\partial \widetilde{\mu}_{0}}{\partial \mu_{0}}-\frac{1}{\widetilde{m}_{K_{0}}^{2}} \frac{\partial \widetilde{m}_{K_{0}}}{\partial \mu_{0}}\left(\frac{3 \bar{I}_{4}^{D} \widetilde{\eta}_{0}^{2}+2 \widetilde{\eta}_{0}\left[\bar{I}_{1} \bar{I}_{5}^{D}+\bar{I}_{4}^{D}-\bar{I}_{6}^{D}\right]+\bar{I}_{1} \bar{I}_{5}^{D}+\bar{I}_{5}^{D}-\bar{I}_{6}^{D}-}{1+\widetilde{\eta}_{0}^{3}+\widetilde{\eta}_{0}^{2} \bar{I}_{2}+\widetilde{\eta}_{0} \bar{I}_{1}}-\right.\right. \\
& \left.\left.\frac{\left(\bar{I}_{5}^{D}+\widetilde{\eta}_{0}^{2} \bar{I}_{4}^{D}+\widetilde{\eta}_{0}\left[\bar{I}_{1} \bar{I}_{5}^{D}-\bar{I}_{6}^{D}\right]\right)\left(\bar{I}_{1}+\widetilde{\eta}_{0}\left[\bar{I}_{1}+2 \bar{I}_{2}\right]+\widetilde{\eta}_{0}^{2}\left[2 \bar{I}_{2}+3\right]+3 \widetilde{\eta}_{0}^{3}\right)}{\left[1+\widetilde{\eta}_{0}^{3}+\widetilde{\eta}_{0}^{2} \bar{I}_{2}+\widetilde{\eta}_{0} \bar{I}_{1}\right]^{2}}\right)\right]+3
\end{aligned}
$$

and

$$
\begin{aligned}
\mathcal{J}_{5}= & \frac{\varepsilon_{0_{\mathrm{p}}}^{2}}{c \widetilde{m}_{K_{0}}^{2}} \frac{\partial \widetilde{m}_{K_{0}}}{\partial \varepsilon_{0_{\mathrm{p}}}}\left[\frac{\bar{I}_{5}^{D}+\bar{I}_{4}^{D} \widetilde{\eta}_{0}^{2}+\widetilde{\eta}_{0}\left[\bar{I}_{1} \bar{I}_{5}^{D}-\bar{I}_{6}^{D}\right]}{1+\widetilde{\eta}_{0}^{3}+\widetilde{\eta}_{0}^{2} \bar{I}_{2}+\widetilde{\eta}_{0} \bar{I}_{1}}\right]+\frac{\varepsilon_{0_{\mathrm{p}}}^{2}}{c \widetilde{m}_{K_{0}}^{2}}\left[\left(1+\widetilde{\eta}_{0}\right) \frac{\partial \widetilde{m}_{K_{0}}}{\partial \varepsilon_{0_{\mathrm{p}}}}-\frac{\partial \widetilde{\varepsilon}_{0}}{\partial \varepsilon_{0_{\mathrm{p}}}}\right] \times \\
& {\left[\frac{\bar{I}_{1} \bar{I}_{5}^{D}-\bar{I}_{6}^{D}+2 \widetilde{\eta}_{0} \bar{I}_{4}^{D}}{1+\widetilde{\eta}_{0}^{3}+\widetilde{\eta}_{0}^{2} \bar{I}_{2}+\widetilde{\eta}_{0} \bar{I}_{1}}-\frac{\left(\bar{I}_{5}^{D}+\widetilde{\eta}_{0}^{2} \bar{I}_{4}^{D}+\widetilde{\eta}_{0}\left[\bar{I}_{1} \bar{I}_{5}^{D}-\bar{I}_{6}^{D}\right]\right)\left(\bar{I}_{1}+2 \widetilde{\eta}_{0} \bar{I}_{2}+3 \widetilde{\eta}_{0}^{2}\right)}{\left[1+\widetilde{\eta}_{0}^{3}+\widetilde{\eta}_{0}^{2} \bar{I}_{2}+\widetilde{\eta}_{0} \bar{I}_{1}\right]^{2}}\right], }
\end{aligned}
$$

where the variables $\mu_{0}$ and $\varepsilon_{0_{\mathrm{p}}}$ are defined implicitly as the solution of the following system of two nonlinear algebraic equations:

$$
\mathcal{F}_{1}^{*}\left\{\mu_{0}, \varepsilon_{0_{\mathrm{p}}}\right\} \doteq \Psi^{\prime}\left(\mathcal{J}_{1}\right)-\frac{\mu_{0}}{2}=0, \quad \mathcal{F}_{2}^{*}\left\{\mu_{0}, \varepsilon_{0_{\mathrm{p}}}\right\} \doteq \mathcal{S}^{*^{\prime}}\left(\mathcal{J}_{5}\right)-\frac{1}{2 \varepsilon_{0_{\mathrm{p}}}}=0 .
$$

Having obtained the approximation (52) for the effective Helmholtz free energy of the composite, the final step of the derivation consist in computing its Legendre transform (44). After making use of the stationarity conditions $\partial \bar{W}^{*} / \partial \mathcal{J}_{1}=\partial \bar{W}^{*} / \partial \mathcal{J}_{5}=\partial \bar{W}^{*} / \partial \mu_{0}=\partial \bar{W}^{*} / \partial \varepsilon_{0_{\mathrm{p}}}=0$ and the convexity properties (22) of the function $\mathcal{S}^{*}$, the result can be written as

$$
\bar{W}(\overline{\mathbf{F}}, \overline{\mathbf{E}}, c)= \begin{cases}(1-c) \Psi\left(\mathcal{I}_{1}\right)-\frac{\mu_{0}(1-c)}{2}\left[\mathcal{I}_{1}-3\right]+\frac{\widetilde{\mu}_{0}}{2}\left[\bar{I}_{1}-3\right]- & \text { if } \bar{J}=1 \\ c \mathcal{S}\left(\mathcal{I}_{5}\right)+\frac{c \varepsilon_{0_{\mathrm{p}}}}{2} \mathcal{I}_{5}+\frac{\widetilde{m}_{K_{0}}-\widetilde{\varepsilon}_{0}}{2} \bar{I}_{4}^{E}-\frac{m_{K_{0}}}{2} \bar{I}_{5}^{E} & \text { otherwise }\end{cases}
$$

with

$$
\mathcal{I}_{1}=\frac{1}{1-c} \frac{\partial \widetilde{\mu}_{0}}{\partial \mu_{0}}\left[\bar{I}_{1}-3\right]+\frac{1}{1-c} \frac{\partial \widetilde{m}_{K_{0}}}{\partial \mu_{0}}\left[\bar{I}_{4}^{E}-\bar{I}_{5}^{E}\right]+3 \text { and } \mathcal{I}_{5}=-\frac{1}{c}\left(\frac{\partial \widetilde{m}_{K_{0}}}{\partial \varepsilon_{0_{\mathrm{p}}}}-\frac{\partial \widetilde{\varepsilon}_{0}}{\partial \varepsilon_{0_{\mathrm{p}}}}\right) \bar{I}_{4}^{E}+\frac{1}{c} \frac{\partial \widetilde{m}_{K_{0}}}{\partial \varepsilon_{0_{\mathrm{p}}}} \bar{I}_{5}^{E}
$$

where, again, $\bar{I}_{1}, \bar{I}_{4}^{E}, \bar{I}_{5}^{E}$ stand for the standard $(\overline{\mathbf{F}}, \overline{\mathbf{E}})$-based invariants defined by $(24)_{1,3,4}, \widetilde{\mu}_{0}, \widetilde{\varepsilon}_{0}, \widetilde{m}_{K_{0}}$ are the effective elastic dielectric coefficients of the comparison medium, as defined by relations (49) with (50)-(51), while the variables $\mu_{0}$ and $\varepsilon_{0_{\mathrm{p}}}$ are defined implicitly as the solution of the system of two nonlinear algebraic equations

$$
\mathcal{F}_{1}\left\{\mu_{0}, \varepsilon_{0_{\mathrm{p}}}\right\} \doteq \Psi^{\prime}\left(\mathcal{I}_{1}\right)-\frac{\mu_{0}}{2}=0, \quad \mathcal{F}_{2}\left\{\mu_{0}, \varepsilon_{0_{\mathrm{p}}}\right\} \doteq \mathcal{S}^{\prime}\left(\mathcal{I}_{5}\right)-\frac{\varepsilon_{0_{\mathrm{p}}}}{2}=0 .
$$


Expression (56) constitutes the main analytical result of this paper: it provides a variational approximation for the effective free energy (4) of a non-Gaussian dielectric elastomer, with free-energy function (10), filled with any type of non-percolative isotropic distribution of nonlinear elastic dielectric particles, with freeenergy function (14). The following remarks are in order.

$i$. The macroscopic constitutive response. In view of the equivalence between equations (57)-(58) and the stationarity conditions $\partial \bar{W} / \partial \mathcal{I}_{1}=\partial \bar{W} / \partial \mathcal{I}_{5}=\partial \bar{W} / \partial \mu_{0}=\partial \bar{W} / \partial \varepsilon_{0_{\mathrm{p}}}=0$, the macroscopic electromechanical constitutive relation (3) implied by the effective free energy (56) is given by

$$
\overline{\mathbf{S}}=\widetilde{\mu}_{0} \overline{\mathbf{F}}+\widetilde{m}_{K_{0}} \overline{\mathbf{F}}^{-T} \overline{\mathbf{E}} \otimes \overline{\mathbf{F}}^{-1} \overline{\mathbf{F}}^{-T} \overline{\mathbf{E}}-\bar{p} \overline{\mathbf{F}}^{-T}
$$

where $\bar{p}$ stands for the arbitrary hydrostatic pressure associated with the incompressibility constraint $\bar{J}=1$, and

$$
\overline{\mathbf{D}}=\left(\widetilde{\varepsilon}_{0}-\widetilde{m}_{K_{0}}\right) \overline{\mathbf{E}}+\widetilde{m}_{K_{0}} \overline{\mathbf{F}}^{-1} \overline{\mathbf{F}}^{-T} \overline{\mathbf{E}} .
$$

Much like the computation of the effective free energy (56) itself, the computation of the macroscopic stress (59) and macroscopic electric displacement (60) for a given microstructure (i.e., a given indicator function $\theta(\mathbf{X})$ ), given constitutive relations for the matrix and particles (i.e., given $\Psi\left(I_{1}\right)$, $\varepsilon_{\mathrm{m}}, \mu_{\mathrm{p}}$, and $\left.\mathcal{S}\left(I_{5}\right)\right)$, and given macroscopic deformation gradient $\overline{\mathbf{F}}$ and macroscopic electric field $\overline{\mathbf{E}}$ amounts to solving the uncoupled linear pdes (50)-(51) for the fields $\boldsymbol{\Gamma}$ and $\boldsymbol{\gamma}$, evaluating the integrals (49) to determine the effective coefficients $\widetilde{\mu}_{0}, \widetilde{\varepsilon}_{0}, \widetilde{m}_{K_{0}}$, and solving the system of nonlinear algebraic equations (58) for $\mu_{0}$ and $\varepsilon_{0_{\mathrm{p}}}$.

ii. The limit of small deformations and moderate electric fields. By construction, the effective free energy (56) is asymptotically exact in the classical limit of small deformations and moderate electric fields. Indeed, in the limit when $\overline{\mathbf{F}} \rightarrow \mathbf{I}$ and $\overline{\mathbf{E}} \rightarrow \mathbf{0}$, the nonlinear algebraic equations (58) admit the explicit solution $\mu_{0}=2 \Psi^{\prime}(3)=\mu$ and $\varepsilon_{0_{\mathrm{p}}}=2 \mathcal{S}^{\prime}(0)=\varepsilon_{\mathrm{p}}$ to leading order, and the effective free energy (56) reduces asymptotically to (see Section 2.1 in Part I)

$$
\bar{W}(\overline{\mathbf{F}}, \overline{\mathbf{E}}, c)= \begin{cases}2 \widetilde{\mu}\left[\left(\bar{\lambda}_{1}-1\right)^{2}+\left(\bar{\lambda}_{2}-1\right)^{2}+\left(\bar{\lambda}_{3}-1\right)^{2}\right]-\frac{\widetilde{\varepsilon}}{2}\left[\bar{E}_{1}^{2}+\bar{E}_{2}^{2}+\bar{E}_{3}^{2}\right]+ & \text { if } \bar{\lambda}_{1} \bar{\lambda}_{2} \bar{\lambda}_{3}=1 \\ \tilde{m}_{K}\left[\left(\bar{\lambda}_{1}-1\right) \bar{E}_{1}^{2}+\left(\bar{\lambda}_{2}-1\right) \bar{E}_{2}^{2}+\left(\bar{\lambda}_{3}-1\right) \bar{E}_{3}^{2}\right] & \text { otherwise } \\ +\infty & \text { other }\end{cases}
$$

Here, $\bar{\lambda}_{1}, \bar{\lambda}_{2}, \bar{\lambda}_{3}$ stand for the singular values of the macroscopic deformation gradient tensor $\overline{\mathbf{F}}$, $\bar{E}_{1}, \bar{E}_{2}, \bar{E}_{3}$ are the components of the macroscopic electric field $\overline{\mathbf{E}}$ with respect to the Lagrangian principal axes (i.e., the principal axes of $\overline{\mathbf{F}}^{T} \overline{\mathbf{F}}$ ), and the effective shear modulus $\widetilde{\mu}$, effective permittivity $\widetilde{\varepsilon}$, and effective electrostrictive constant $\widetilde{m}_{K}$ are defined by relations (49) with (50)-(51) when evaluated at the aforementioned solution of equations (58), namely, $\mu_{0}=\mu$ and $\varepsilon_{0_{\mathrm{p}}}=\varepsilon_{\mathrm{p}}$.

iii. Independence of $\bar{W}$ on the invariants $\bar{I}_{2}$ and $\bar{I}_{6}^{E}$. For finite deformations and finite electric fields, the effective free energy (56) is in general not exact. The direct comparisons with FE simulations presented further below in Section 6 support that it does provide, however, an accurate approximation. In this regard, we remark in particular that the effective free energy (56) is independent of the invariants $\bar{I}_{2}$ and $\bar{I}_{6}^{E}$. The FE simulations presented in Section 6 indicate that this distinctive functional trait is indeed exhibited by the corresponding exact solutions.

$i v$. The case of ideal elastic dielectric constituents. For finite deformations and finite electric fields, the nonlinear algebraic equations (58) do not generally admit explicit solutions. There are, nonetheless, a number of special cases of practical interest for which they do. The first one that we report here corresponds to the basic case when both the matrix and the particles are ideal elastic dielectrics, which was the focus of Part I. Namely, when

$$
\Psi\left(I_{1}\right)=\frac{\mu}{2}\left[I_{1}-3\right] \quad \text { and } \quad \mathcal{S}\left(I_{5}^{E}\right)=\frac{\varepsilon_{\mathrm{p}}}{2} I_{5}^{E},
$$


the equations (58) are solved by $\mu_{0}=\mu$ and $\varepsilon_{0_{\mathrm{p}}}=\varepsilon_{\mathrm{p}}$. In this case, the effective free energy (56) reduces, of course, to the result obtained in Part I:

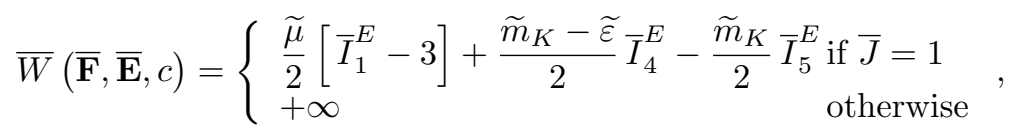

where, as in the asymptotic result (61), the effective constants $\widetilde{\mu}, \widetilde{\varepsilon}, \widetilde{m}_{K}$ are defined by relations (49) with (50)-(51) when $\mu_{0}=\mu$ and $\varepsilon_{0_{\mathrm{p}}}=\varepsilon_{\mathrm{p}}$.

$v$. The limiting case of rigid particles: $\mu_{\mathrm{p}}=+\infty$. The majority of existing experimental investigations on dielectric elastomer composites involve filler particles that are mechanically much stiffer than the underlying elastomeric matrix. For example, PANI, O-CuPc, and $\mathrm{TiO}_{2}$ particles, which have been often utilized in experimental investigations (see, e.g., Li et al., 2004; Huang et al., 2005; Liu et al., 2013), all exhibit initial shear moduli that are several orders of magnitude larger than the shear moduli of typical dielectric elastomers, such as for instance acrylic elastomers and silicones. This prompts the analysis of the effective free energy (56) in the limit of rigid particles when $\mu_{\mathrm{p}}=+\infty$.

Now, for the case of rigid particles when $\mu_{\mathrm{p}}=+\infty$, the effective shear modulus $\widetilde{\mu}_{0}$ of the comparison medium is necessarily linear in $\mu_{0}$, while the effective electrostrictive coefficient $\widetilde{m}_{K_{0}}$ is independent of $\mu_{0}$. More specifically, we have that

$$
\widetilde{\mu}_{0}=(1-c) r(c) \mu_{0} \quad \text { and } \quad \frac{\partial \widetilde{m}_{K_{0}}}{\partial \mu_{0}}=0
$$

with

$$
r(c)=\frac{1}{5(1-c)} \int_{\Omega}[1-\theta(\mathbf{X})] \mathcal{K}_{k l m n} \Gamma_{m k l, n} \mathrm{~d} \mathbf{X}
$$

where the field $\boldsymbol{\Gamma}$ here corresponds to the solution of the linear boundary value problem (50) for the choice of particle shear modulus $\mu_{\mathrm{p}}=+\infty$. It follows from (64) that equation $(58)_{1}$ can be solved in closed form for $\mu_{0}$. The result reads as $\mu_{0}=2 \Psi^{\prime}\left(r(c)\left[\bar{I}_{1}-3\right]+3\right)$. In turn, the effective free energy (56) simplifies to

$\bar{W}(\overline{\mathbf{F}}, \overline{\mathbf{E}}, c)=\left\{\begin{array}{l}(1-c) \Psi\left(r(c)\left[\bar{I}_{1}-3\right]+3\right)-c \mathcal{S}\left(\mathcal{I}_{5}\right)+\frac{c \varepsilon_{0_{\mathrm{p}}}}{2} \mathcal{I}_{5}+\frac{\widetilde{m}_{K_{0}}-\widetilde{\varepsilon}_{0}}{2} \bar{I}_{4}^{E}-\frac{\widetilde{m}_{K_{0}}}{2} \bar{I}_{5}^{E} \text { if } \bar{J}=1 \\ +\infty\end{array}\right.$, otherwise

where we recall that the variable $\mathcal{I}_{5}$ is given by expression $(57)_{2}, \widetilde{\varepsilon}_{0}$ and $\widetilde{m}_{K_{0}}$ are the effective permittivity and electrostrictive coefficient of the comparison medium, as defined by relations $(49)_{2}$ and $(49)_{3}$ with (50)-(51) for the case when $\mu_{0}=2 \Psi^{\prime}\left(r(c)\left[\bar{I}_{1}-3\right]+3\right)$ and $\mu_{\mathrm{p}}=+\infty$, while the variable $\varepsilon_{0_{\mathrm{p}}}$ is defined implicitly as the solution of the nonlinear algebraic equation $(58)_{2}$.

In the absence of an electric field when $\overline{\mathbf{E}}=\mathbf{0}$, it is fitting to mention that equation (58) $)_{2}$ is solved by $\varepsilon_{0_{\mathrm{p}}}=\varepsilon_{\mathrm{p}}$ and the effective free-energy function (66) reduces to an earlier result of Lopez-Pamies et al. (2013) for the effective stored-energy function of an isotropic suspension of rigid inclusions in non-Gaussian rubber:

$$
\bar{W}(\overline{\mathbf{F}}, \mathbf{0}, c)=\left\{\begin{array}{ll}
(1-c) \Psi\left(r(c)\left[\bar{I}_{1}-3\right]+3\right) & \text { if } \bar{J}=1 \\
+\infty & \text { otherwise }
\end{array} .\right.
$$

vi. The limiting case of liquid-like particles: $\mu_{\mathrm{p}}=0$. Recent theoretical (Lopez-Pamies, 2014; Spinelli et al., 2015) and experimental (Fassler and Majidi, 2015) investigations have indicated that the addition of liquid-like filler particles — that is, incompressible particles of vanishingly small shear resistance - to dielectric elastomers may have the potential to lead to significantly enhanced elastic dielectric properties.

In the present context, liquid-like particles can be modelled by setting $\mu_{\mathrm{p}}=0$. In this limiting case, much like for rigid particles, the effective shear modulus $\widetilde{\mu}_{0}$ of the comparison medium can be shown 
to depend linearly on $\mu_{0}$, while the effective electrostrictive coefficient $\widetilde{m}_{K_{0}}$ is independent altogether of the value of $\mu_{0}$. In particular, we have that

$$
\widetilde{\mu}_{0}=(1-c) l(c) \mu_{0} \quad \text { and } \quad \frac{\partial \widetilde{m}_{K_{0}}}{\partial \mu_{0}}=0
$$

with

$$
l(c)=\frac{1}{5(1-c)} \int_{\Omega}[1-\theta(\mathbf{X})] \mathcal{K}_{k l m n} \Gamma_{m k l, n} \mathrm{~d} \mathbf{X},
$$

where the field $\boldsymbol{\Gamma}$ in this last expression corresponds to the solution of the linear boundary value problem (50) for the choice of particle shear modulus $\mu_{\mathrm{p}}=0$. Given relations (68), it is a simple matter to recognize that equation (58) $)_{1}$ can be solved in closed form for the variable $\mu_{0}$. The result reads as $\mu_{0}=2 \Psi^{\prime}\left(l(c)\left[\bar{I}_{1}-3\right]+3\right)$. This in turn leads to the following simplification of the effective free energy (56):

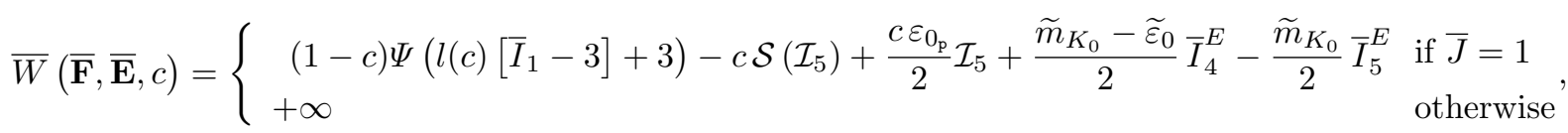

where, again, the variable $\mathcal{I}_{5}$ is given by expression $(57)_{2}, \widetilde{\varepsilon}_{0}$ and $\widetilde{m}_{K_{0}}$ are the effective permittivity and electrostrictive coefficient of the comparison medium, as defined by relations $(49)_{2}$ and $(49)_{3}$ with (50)-(51) for the case when $\mu_{0}=2 \Psi^{\prime}\left(l(c)\left[\bar{I}_{1}-3\right]+3\right)$ and $\mu_{\mathrm{p}}=0$, while the variable $\varepsilon_{0_{\mathrm{p}}}$ is defined implicitly by equation $(58)_{2}$.

vii. The $\overline{\mathbf{F}}$ and $\overline{\mathbf{D}}$ formulation. With help of the effective Helmholtz free energy (52) and relations (8), it is straightforward to partly invert the macroscopic constitutive relations (59)-(60) in order to rewrite them with the electric displacement field $\overline{\mathbf{D}}$ playing the role of electric independent variable instead of the electric field $\overline{\mathbf{E}}$. The result reads as

$$
\begin{aligned}
\overline{\mathbf{S}}= & \widetilde{\mu}_{0} \overline{\mathbf{F}}-\bar{q} \overline{\mathbf{F}}^{-T}-\frac{\widetilde{\eta}_{0} \bar{I}_{5}^{D}+\widetilde{\eta}_{0}^{3} \bar{I}_{4}^{D}+\widetilde{\eta}_{0}^{2}\left[\bar{I}_{1} \bar{I}_{5}^{D}-\bar{I}_{6}^{D}\right]}{\widetilde{m}_{K_{0}}\left[1+\widetilde{\eta}_{0}^{3}+\widetilde{\eta}_{0}^{2} \bar{I}_{2}+\widetilde{\eta}_{0} \bar{I}_{1}\right]^{2}}\left[\overline{\mathbf{F}}-\widetilde{\eta}_{0} \overline{\mathbf{F}}^{-T} \overline{\mathbf{F}}^{-1} \overline{\mathbf{F}}^{-T}\right]+ \\
& \frac{1}{\widetilde{m}_{K_{0}}\left[1+\widetilde{\eta}_{0}^{3}+\widetilde{\eta}_{0}^{2} \bar{I}_{2}+\widetilde{\eta}_{0} \bar{I}_{1}\right]} \times\left(\widetilde{\eta}_{0}\left[\bar{I}_{5}^{D} \overline{\mathbf{F}}-\overline{\mathbf{F}} \overline{\mathbf{D}} \otimes \overline{\mathbf{F}}^{T} \overline{\mathbf{F}} \overline{\mathbf{D}}-\overline{\mathbf{F}} \overline{\mathbf{F}}^{T} \overline{\mathbf{F}} \overline{\mathbf{D}} \otimes \overline{\mathbf{D}}\right]+\left(1+\bar{I}_{1} \widetilde{\eta}_{0}\right) \overline{\mathbf{F}} \overline{\mathbf{D}} \otimes \overline{\mathbf{D}}\right),
\end{aligned}
$$

where we recall that $\widetilde{\eta}_{0}=\left(\widetilde{\varepsilon}_{0}-\widetilde{m}_{K_{0}}\right) / \widetilde{m}_{K_{0}}, \bar{q}$ stands for the arbitrary hydrostatic pressure associated with the incompressibility constraint $\bar{J}=1$, and

$$
\overline{\mathbf{E}}=\frac{1}{\widetilde{m}_{K_{0}}\left[1+\widetilde{\eta}_{0}^{3}+\widetilde{\eta}_{0}^{2} \bar{I}_{2}+\widetilde{\eta}_{0} \bar{I}_{1}\right]}\left[\left(1+\bar{I}_{1} \widetilde{\eta}_{0}\right) \overline{\mathbf{F}}^{T} \overline{\mathbf{F}} \overline{\mathbf{D}}+\widetilde{\eta}_{0}^{2} \overline{\mathbf{D}}-\widetilde{\eta}_{0} \overline{\mathbf{F}}^{T} \overline{\mathbf{F}} \overline{\mathbf{F}}^{T} \overline{\mathbf{F}} \overline{\mathbf{D}}\right] .
$$

viii. Material instabilities. In addition to facilitating the computation of the macroscopic electromechanical constitutive response (71)-(72), the effective Helmholtz free-energy function (52) in terms of $\overline{\mathbf{F}}$ and $\overline{\mathbf{D}}$ provides the means to conveniently determine the possible onset of two classes of material instabilities: $i$ ) instabilities associated with electromechanical limit loads and ii) microstructural instabilities of long wavelength. Section 5.2 of Part I discusses the relevant conditions that signal the triggering of these instabilities and hence we do not repeat them here.

As already indicated above, the variational solution (56) applies to arbitrary non-percolative isotropic distributions of filler particles. With the two-pronged objective of demonstrating its use and of facilitating comparisons with experimental results, in the next two subsections we present the specialization of the approximation (56) to two specific types of isotropic distributions of filler particles: $i$ ) an isotropic iterative microstructure wherein the particles are infinitely polydisperse in size and $i i$ ) an isotropic distribution of monodisperse spherical particles. For conciseness, we shall restrict attention to the practically relevant cases of rigid and liquid-like filler particles. 


\subsection{An isotropic iterative microstructure with infinitely polydisperse particles}

We begin with the specialization of (56) to the iterative microstructure of Lopez-Pamies (2014), wherein the filler particles are infinitely polydisperse in size. In a recent contribution, Spinelli et al. (2015) worked out exact closed-form solutions for the effective elastic dielectric tensors that characterize the response of dielectric elastomer composites with such iterative microstructures in the limit of small deformations and moderate electric fields. In its general form, their solution applies to anisotropic microstructures, as well as to compressible and anisotropic matrix and filler particle behaviors. In the sequel, we invoke its specialization to the two cases of interest here: incompressible matrix materials and isotropic distributions of rigid and liquid-like particles.

Rigid particles. When specialized to incompressible dielectric elastomers with initial shear modulus $\mu_{0}$ and initial permittivity $\varepsilon$ and to isotropic distributions of rigid particles with initial shear modulus $\mu_{\mathrm{p}}=+\infty$ and initial permittivity $\varepsilon_{0_{\mathrm{p}}}$, the result of Spinelli et al. (2015) for the effective elastic dielectric coefficients (49) reduces to

$$
\begin{aligned}
& \widetilde{\mu}_{0}=\mu_{0}+\frac{5 c}{2(1-c)} \mu_{0}, \quad \widetilde{\varepsilon}_{0}=\varepsilon+\frac{3 c\left(\varepsilon_{0_{\mathrm{p}}}-\varepsilon\right)}{(2+c) \varepsilon+(1-c) \varepsilon_{0_{\mathrm{p}}}} \varepsilon, \\
& \widetilde{m}_{K_{0}}=\varepsilon+\frac{3 c\left(\varepsilon_{0_{\mathrm{p}}}-\varepsilon\right)\left[(23+7 c) \varepsilon+7(1-c) \varepsilon_{0_{\mathrm{p}}}\right]}{10\left[(2+c) \varepsilon+(1-c) \varepsilon_{0_{\mathrm{p}}}\right]^{2}} \varepsilon .
\end{aligned}
$$

It is trivial to deduce from $(73)_{1}$ that the corresponding function $r(c)$ defined by $(65)$ is thus given by

$$
r(c)=\frac{2+3 c}{2(1-c)^{2}}
$$

Moreover, it is a simple matter to deduce that the finite branch of the effective free-energy function (66) for this class of dielectric elastomer composites specializes to

$$
\bar{W}(\overline{\mathbf{F}}, \overline{\mathbf{E}}, c)=(1-c) \Psi\left(\frac{2+3 c}{2(1-c)^{2}}\left[\bar{I}_{1}-3\right]+3\right)-c \mathcal{S}\left(\mathcal{I}_{5}^{\mathrm{HJ}}\right)+\frac{c \varepsilon_{0_{\mathrm{p}}}}{2} \mathcal{I}_{5}^{\mathrm{HJ}_{r}}+\frac{\widetilde{m}_{K_{0}}-\widetilde{\varepsilon}_{0}}{2} \bar{I}_{4}^{E}-\frac{\widetilde{m}_{K_{0}}}{2} \bar{I}_{5}^{E}
$$

where $\widetilde{\varepsilon}_{0}$ and $\widetilde{m}_{K_{0}}$ are given by expressions $(73)_{2,3}$,

$$
\mathcal{I}_{5}^{\mathrm{HJ}}=\frac{27(1-c)\left(\varepsilon_{0_{\mathrm{p}}}-\varepsilon\right) \varepsilon^{2}}{5\left[(2+c) \varepsilon+(1-c) \varepsilon_{0_{\mathrm{p}}}\right]^{3}} \bar{I}_{4}^{E}+\frac{9\left[(13+2 c) \varepsilon+2(1-c) \varepsilon_{0_{\mathrm{p}}}\right] \varepsilon^{2}}{5\left[(2+c) \varepsilon+(1-c) \varepsilon_{0_{\mathrm{p}}}\right]^{3}} \bar{I}_{5}^{E},
$$

and the variable $\varepsilon_{0_{\mathrm{p}}}$ is defined as the solution of the nonlinear algebraic equation

$$
\mathcal{S}^{\prime}\left(\mathcal{I}_{5}^{\mathrm{HJ}_{r}}\right)-\frac{\varepsilon_{0_{\mathrm{p}}}}{2}=0
$$

which, in general, for most choices of the function $\mathcal{S}$, needs to be solved numerically.

The effective free-energy function (75) contains several limiting cases of practical significance. Here, we report the case corresponding to filler particles that, in addition to being infinitely stiff, are electrically conducting so that the function $\mathcal{S}\left(I_{5}^{E}\right)=0$ if $I_{5}^{E}=0$ and $\mathcal{S}\left(I_{5}^{E}\right)=+\infty$ otherwise. For such a limiting case, the solution of equation (77) can be shown to be given by $\varepsilon_{0_{\mathrm{p}}}=+\infty$ and the effective free-energy function (75) simplifies to the following fully explicit expression:

$$
\bar{W}(\overline{\mathbf{F}}, \overline{\mathbf{E}}, c)=(1-c) \Psi\left(\frac{2+3 c}{2(1-c)^{2}}\left[\bar{I}_{1}-3\right]+3\right)-\frac{9 c \varepsilon}{20(1-c)} \bar{I}_{4}^{E}-\frac{(10+11 c) \varepsilon}{20(1-c)} \bar{I}_{5}^{E} .
$$

The result (78) is relevant for dielectric elastomer composites wherein the filler particles are hard (semi)conducting polymers (e.g., PANI and $\mathrm{O}-\mathrm{CuPc}$ ) or metals. 
Liquid-like particles. When specialized to incompressible dielectric elastomers with initial shear modulus $\mu_{0}$ and initial permittivity $\varepsilon$ and to isotropic distributions of liquid-like particles with initial shear modulus $\mu_{\mathrm{p}}=0$ and initial permittivity $\varepsilon_{0_{\mathrm{p}}}$, the result of Spinelli et al. (2015) for the effective elastic dielectric coefficients (49) reduces to

$$
\begin{aligned}
& \widetilde{\mu}_{0}=\mu_{0}-\frac{5 c}{3+2 c} \mu_{0}, \widetilde{\varepsilon}_{0}=\varepsilon+\frac{3 c\left(\varepsilon_{0_{\mathrm{p}}}-\varepsilon\right)}{(2+c) \varepsilon+(1-c) \varepsilon_{0_{\mathrm{p}}}} \varepsilon, \\
& \widetilde{m}_{K_{0}}=\varepsilon+\frac{3 c\left(\varepsilon_{0_{\mathrm{p}}}-\varepsilon\right)\left[\left(42+26 c+7 c^{2}\right) \varepsilon+(1-c)(3+7 c) \varepsilon_{0_{\mathrm{p}}}\right]}{5(3+2 c)\left[(2+c) \varepsilon+(1-c) \varepsilon_{0_{\mathrm{p}}}\right]^{2}} \varepsilon .
\end{aligned}
$$

The corresponding function $l(c)$ defined by (69) is given by

$$
l(c)=\frac{3}{3+2 c} .
$$

In view of the above expressions, the finite branch of the effective free-energy function (70) for this class of dielectric elastomer composites reduces to

$$
\bar{W}(\overline{\mathbf{F}}, \overline{\mathbf{E}}, c)=(1-c) \Psi\left(\frac{3}{3+2 c}\left[\bar{I}_{1}-3\right]+3\right)-c \mathcal{S}\left(\mathcal{I}_{5}^{\mathrm{HJ}}{ }_{l}\right)+\frac{c \varepsilon_{0_{\mathrm{p}}}}{2} \mathcal{I}_{5}^{\mathrm{HJ}_{l}}+\frac{\widetilde{m}_{K_{0}}-\widetilde{\varepsilon}_{0}}{2} \bar{I}_{4}^{E}-\frac{\widetilde{m}_{K_{0}}}{2} \bar{I}_{5}^{E}
$$

where $\widetilde{\varepsilon}_{0}$ and $\widetilde{m}_{K_{0}}$ are given by expressions $(79)_{2,3}$,

$$
\mathcal{I}_{5}^{\mathrm{HJ}_{l}}=\frac{54(1-c)(4+c)\left(\varepsilon_{0_{\mathrm{p}}}-\varepsilon\right) \varepsilon^{2}}{5(3+2 c)\left[(2+c) \varepsilon+(1-c) \varepsilon_{0_{\mathrm{p}}}\right]^{3}} \bar{I}_{4}^{E}+\frac{9\left[\left(54+17 c+4 c^{2}\right) \varepsilon-(1-c)(9-4 c) \varepsilon_{0_{\mathrm{p}}}\right] \varepsilon^{2}}{5(3+2 c)\left[(2+c) \varepsilon+(1-c) \varepsilon_{0_{\mathrm{p}}}\right]^{3}} \bar{I}_{5}^{E},
$$

and $\varepsilon_{0_{\mathrm{p}}}$ is defined implicitly by the nonlinear algebraic equation

$$
\mathcal{S}^{\prime}\left(\mathcal{I}_{5}^{\mathrm{HJ}_{l}}\right)-\frac{\varepsilon_{0_{\mathrm{p}}}}{2}=0
$$

which, similar to (77), for most choices of the function $\mathcal{S}$, needs to be solved numerically.

For the limiting case when the particles, in addition to being of vanishingly small shear resistance, are electrically conducting, the solution of equation (83) can be shown to be given by $\varepsilon_{0_{\mathrm{p}}}=+\infty$ and the effective free-energy function (81) reduces to

$$
\bar{W}(\overline{\mathbf{F}}, \overline{\mathbf{E}}, c)=(1-c) \Psi\left(\frac{3}{3+2 c}\left[\bar{I}_{1}-3\right]+3\right)-\frac{9 c(4+c) \varepsilon}{10\left(3-c-2 c^{2}\right)} \bar{I}_{4}^{E}-\frac{\left(15+4 c+11 c^{2}\right) \varepsilon}{10\left(3-c-2 c^{2}\right)} \bar{I}_{5}^{E} .
$$

The simple explicit result (84) is relevant for dielectric elastomer composites wherein the filler particles are conducting liquids (e.g., Galistan).

\subsection{An isotropic distribution of monodisperse spherical particles}

Complementary to the foregoing results, in this subsection we present the specialization of the effective free energy (56) to an isotropic distribution of monodisperse spherical particles.

Now, for an isotropic distribution of monodisperse spherical particles, the pdes (50) and (51) do not admit an analytical solution. As discussed in Appendix A of Spinelli et al. (2015), however, they can be readily solved numerically by means of finite elements (FE). This in turn allows for the numerical evaluation of the effective coefficients (49) required in the computation of the effective free energy (56). In general, since the derivatives $\partial \widetilde{\mu}_{0} / \partial \mu_{0}, \partial \widetilde{m}_{K_{0}} / \partial \mu_{0}, \partial \widetilde{\varepsilon}_{0} / \partial \varepsilon_{0_{\mathrm{p}}}, \partial \widetilde{m}_{K_{0}} / \partial \varepsilon_{0_{\mathrm{p}}}$ of the effective coefficients (49) are also needed in the computation of (56), the pdes (50) and (51) need to be solved multiple times for a sufficiently wide range of values $\mu_{0}>0$ and $\varepsilon_{0_{\mathrm{p}}} \geq \varepsilon_{0}$ to make possible the numerical computation of these derivatives. For the two specific cases of interest here when the filler particles are either rigid or liquid-like, the pde (50) needs to be solved only once since it is independent of $\mu_{0}$ (see remarks $v$ and $v i$ above) and only the pde (51) needs to be solved multiple times for a sufficiently wide range of values $\varepsilon_{0_{\mathrm{p}}} \geq \varepsilon_{0}$. For these two cases, moreover, it is possible to obtain from the FE solutions simple explicit interpolating formulas for the effective elastic dielectric coefficients (49) over a large range of volume fractions of particles $c$. In the sequel, we report such formulas for the practically relevant range $c \in[0,0.25]$. 
Rigid particles. For incompressible dielectric elastomers, with initial shear modulus $\mu_{0}$ and initial permittivity $\varepsilon$, and rigid spherical particles, with initial shear modulus $\mu_{\mathrm{p}}=+\infty$ and initial permittivity $\varepsilon_{0_{\mathrm{p}}}$, the FE solutions for the effective elastic dielectric coefficients (49) are accurately described for the range of volume fraction of particles $c \in[0,0.25]$ by the following explicit formulas:

$$
\begin{aligned}
& \widetilde{\mu}_{0}=\frac{\mu_{0}}{(1-c)^{5 / 2}}, \quad \widetilde{\varepsilon}_{0}=\varepsilon+\frac{3 c \varepsilon\left(\varepsilon_{0_{\mathrm{p}}}-\varepsilon\right)}{(2+c) \varepsilon+(1-c) \varepsilon_{0_{\mathrm{p}}}}, \\
& \widetilde{m}_{K_{0}}=\varepsilon+\frac{3 c \varepsilon\left(\varepsilon_{0_{\mathrm{p}}}-\varepsilon\right)\left[\left(10+2 c+3 c^{2}\right) \varepsilon+(1-c)(5+3 c) \varepsilon_{0_{\mathrm{p}}}\right]}{5\left[(2+c) \varepsilon+(1-c) \varepsilon_{0_{\mathrm{p}}}\right]^{2}} .
\end{aligned}
$$

The corresponding function $r(c)$ defined by (65) is given by

$$
r(c)=\frac{1}{(1-c)^{7 / 2}} .
$$

In view of relations (85)-(86), the finite branch of the effective free-energy function (66) for this class of dielectric elastomer composites specializes to

$$
\bar{W}(\overline{\mathbf{F}}, \overline{\mathbf{E}}, c)=(1-c) \Psi\left(\frac{\bar{I}_{1}-3}{(1-c)^{7 / 2}}+3\right)-c \mathcal{S}\left(\mathcal{I}_{5}^{\mathrm{Sph}_{r}}\right)+\frac{c \varepsilon_{0_{\mathrm{p}}}}{2} \mathcal{I}_{5}^{\mathrm{Sph}_{r}}+\frac{\widetilde{m}_{K_{0}}-\widetilde{\varepsilon}_{0}}{2} \bar{I}_{4}^{E}-\frac{\tilde{m}_{K_{0}}}{2} \bar{I}_{5}^{E},
$$

where $\widetilde{\varepsilon}_{0}$ and $\widetilde{m}_{K_{0}}$ are given by expressions $(85)_{2,3}$,

$$
\mathcal{I}_{5}^{\mathrm{Sph}_{r}}=-\frac{54 c(1-c)\left(\varepsilon_{0_{\mathrm{p}}}-\varepsilon\right) \varepsilon^{2}}{5\left[(2+c) \varepsilon+(1-c) \varepsilon_{0_{\mathrm{p}}}\right]^{3}} \bar{I}_{4}^{E}+\frac{9\left[\left(10-c+6 c^{2}\right) \varepsilon+\left(5+c-6 c^{2}\right) \varepsilon_{0_{\mathrm{p}}}\right] \varepsilon^{2}}{5\left[(2+c) \varepsilon+(1-c) \varepsilon_{0_{\mathrm{p}}}\right]^{3}} \bar{I}_{5}^{E},
$$

and the variable $\varepsilon_{0_{\mathrm{p}}}$ is defined implicitly by the nonlinear algebraic equation

$$
\mathcal{S}^{\prime}\left(\mathcal{I}_{5}^{\operatorname{Sph}_{r}}\right)-\frac{\varepsilon_{0_{\mathrm{p}}}}{2}=0 .
$$

For the limiting case when the particles are electrically conducting, equation (89) admits the explicit solution $\varepsilon_{0_{\mathrm{p}}}=+\infty$ and the effective free-energy function (87) reduces to

$$
\bar{W}(\overline{\mathbf{F}}, \overline{\mathbf{E}}, c)=(1-c) \Psi\left(\frac{\bar{I}_{1}-3}{(1-c)^{7 / 2}}+3\right)+\frac{9 c^{2} \varepsilon}{10(1-c)} \bar{I}_{4}^{E}-\frac{\left(5+10 c+9 c^{2}\right) \varepsilon}{10(1-c)} \bar{I}_{5}^{E} .
$$

Again, much like the effective free-energy function (78), the fully explicit effective free-energy function (90) is relevant for dielectric elastomer composites wherein the filler particles are hard (semi-)conducting materials.

Liquid-like particles. For incompressible dielectric elastomers, with initial shear modulus $\mu_{0}$ and initial permittivity $\varepsilon$, and liquid-like spherical particles, with initial shear modulus $\mu_{\mathrm{p}}=0$ and initial permittivity $\varepsilon_{0_{\mathrm{p}}}$, the FE solutions for the effective elastic dielectric coefficients (49) are accurately described for the range of volume fraction of particles $c \in[0,0.25]$ by the following formulas:

$$
\begin{aligned}
\widetilde{\mu}_{0} & =(1-c)^{5 / 3} \mu_{0}, \quad \widetilde{\varepsilon}_{0}=\varepsilon+\frac{3 c \varepsilon\left(\varepsilon_{0_{\mathrm{p}}}-\varepsilon\right)}{(2+c) \varepsilon+(1-c) \varepsilon_{0_{\mathrm{p}}}}, \\
\widetilde{m}_{K_{0}} & =\varepsilon+\frac{9 \varepsilon\left(\varepsilon_{0_{\mathrm{p}}}-\varepsilon\right)^{2}}{\left[(2+c) \varepsilon+(1-c) \varepsilon_{0_{\mathrm{p}}}\right]^{2}}\left(\frac{4 c^{2}}{45}-\frac{81 c^{61 / 25}}{500}+\frac{c \varepsilon}{\varepsilon_{0_{\mathrm{p}}}-\varepsilon}\right) .
\end{aligned}
$$

The corresponding function $l(c)$ defined by (69) is given by

$$
l(c)=(1-c)^{2 / 3} .
$$

It follows from expressions (91)-(92) that the finite branch of the effective free-energy function (70) for this class of dielectric elastomer composites reduces to

$$
\bar{W}(\overline{\mathbf{F}}, \overline{\mathbf{E}}, c)=(1-c) \Psi\left((1-c)^{2 / 3}\left[I_{1}-3\right]+3\right)-c \mathcal{S}\left(\mathcal{I}_{5}^{\mathrm{Sph}_{l}}\right)+\frac{c \varepsilon_{0_{\mathrm{p}}}}{2} \mathcal{I}_{5}^{\mathrm{Sph}_{l}}+\frac{\widetilde{m}_{K_{0}}-\widetilde{\varepsilon}_{0}}{2} \bar{I}_{4}^{E}-\frac{\widetilde{m}_{K_{0}}}{2} \bar{I}_{5}^{E}
$$


where $\widetilde{\varepsilon}_{0}$ and $\widetilde{m}_{K_{0}}$ are given by expressions $(91)_{2,3}$,

$$
\mathcal{I}_{5}^{\mathrm{Sph}_{l}}=\frac{3\left(1500-1900 c+729 c^{36 / 25}\right)\left(\varepsilon_{0_{\mathrm{p}}}-\varepsilon\right) \varepsilon^{2}}{250\left[(2+c) \varepsilon+(1-c) \varepsilon_{0_{\mathrm{p}}}\right]^{3}} \bar{I}_{4}^{E}-\frac{3\left[\left(750-1150 c+729 c^{36 / 25}\right)\left(\varepsilon_{0_{\mathrm{p}}}-\varepsilon\right)-2250 \varepsilon\right] \varepsilon^{2}}{250\left[(2+c) \varepsilon+(1-c) \varepsilon_{0_{\mathrm{p}}}\right]^{3}} \bar{I}_{5}^{E},
$$

and $\varepsilon_{0_{\mathrm{p}}}$ is implicitly defined by the nonlinear algebraic equation

$$
\mathcal{S}^{\prime}\left(\mathcal{I}_{5}^{\mathrm{Sph}_{l}}\right)-\frac{\varepsilon_{0_{\mathrm{p}}}}{2}=0
$$

For the limiting case when the particles, in addition to being of vanishingly small shear resistance, are electrically conducting, the solution of equation (95) is simply given by $\varepsilon_{0_{\mathrm{p}}}=+\infty$ and the effective free-energy function (93) simplifies to

$$
\begin{aligned}
\bar{W}(\overline{\mathbf{F}}, \overline{\mathbf{E}}, c)= & (1-c) \Psi\left((1-c)^{2 / 3}\left[\bar{I}_{1}-3\right]+3\right)-\frac{c\left(1500-1900 c+729 c^{36 / 25}\right) \varepsilon}{1000(1-c)^{2}} \bar{I}_{4}^{E}- \\
& \frac{\left(500-1000 c+900 c^{2}-729 c^{61 / 25}\right) \varepsilon}{1000(1-c)^{2}} \bar{I}_{5}^{E} .
\end{aligned}
$$

Akin to the effective free-energy function (84), the fully explicit effective free-energy function (96) is relevant for dielectric elastomer composites wherein the fillers are conducting liquids.

\subsection{Electrostriction}
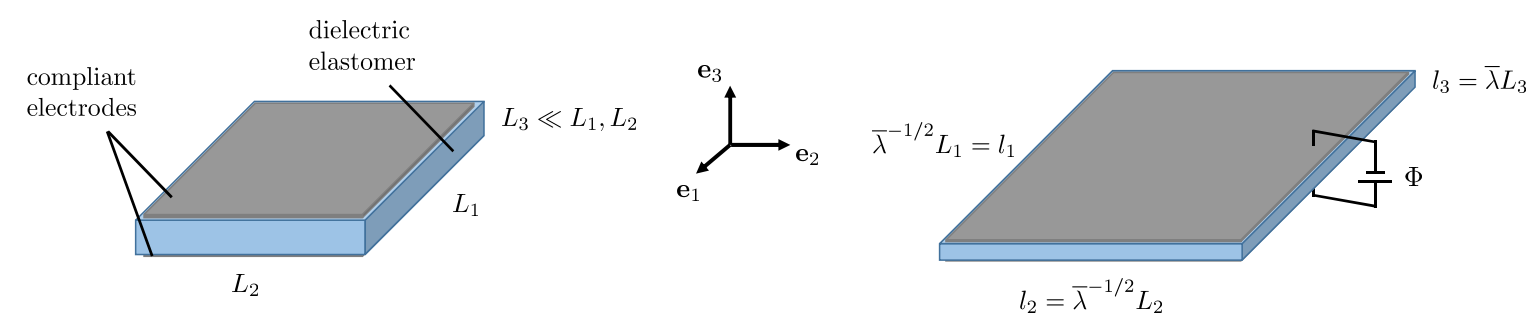

Figure 1: Schematic of the typical experimental setup, in (left) the undeformed and (right) the deformed configurations, to probe the electrostriction of dielectric elastomers under the application of an uniaxial Lagrangian electric field $\bar{E}=-\Phi / L_{3}$.

We conclude this section by spelling out the response predicted by the effective free-energy function (56) for the experimentally prominent boundary conditions of uniaxial electrostriction when the macroscopic first Piola-Kirchhoff stress $\overline{\mathbf{S}}$ and macroscopic electric field $\overline{\mathbf{E}}$ are of the form

$$
\bar{S}_{i j}=\left[\begin{array}{ccc}
0 & 0 & 0 \\
0 & 0 & 0 \\
0 & 0 & 0
\end{array}\right], \quad \bar{E}_{i}=\left[\begin{array}{c}
0 \\
0 \\
\bar{E}
\end{array}\right]
$$

the components of all tensorial quantities throughout this subsection are referred to the Cartesian laboratory axes $\mathbf{e}_{1}, \mathbf{e}_{2}, \mathbf{e}_{3}$ depicted in Fig. 1. It follows from the constitutive relations (59) and (60) that

$$
\bar{F}_{i j}=\left[\begin{array}{ccc}
\bar{\lambda}^{-1 / 2} & 0 & 0 \\
0 & \bar{\lambda}^{-1 / 2} & 0 \\
0 & 0 & \bar{\lambda}
\end{array}\right] \quad \text { and } \quad \bar{D}_{i}=\left[\begin{array}{c}
0 \\
0 \\
\bar{D}
\end{array}\right]
$$

where the electrostriction stretch $\bar{\lambda}$ in the direction of the applied electric field (see Fig. 1) and the non-trivial component $\bar{D}$ of the electric displacement field are defined by the relations

$$
\bar{\lambda}^{4}-\bar{\lambda}+\frac{\widetilde{m}_{K_{0}}}{\widetilde{\mu}_{0}} \bar{E}^{2}=0 \quad \text { and } \quad \bar{D}=\left[\widetilde{\varepsilon}_{0}-\widetilde{m}_{K_{0}}\left(1-\frac{1}{\bar{\lambda}^{2}}\right)\right] \bar{E}
$$


in terms of the applied electric field $\bar{E}$. Here, we stress yet again that the coefficients $\widetilde{\mu}_{0}, \widetilde{\varepsilon}_{0}, \widetilde{m}_{K_{0}}$ in (99) are ultimately functions of the microstructure through the indicator function $\theta(\mathbf{X})$, of the properties of the dielectric elastomeric matrix through the function $\Psi$ and initial permittivity $\varepsilon$, of the properties of the filler particles through the initial shear modulus $\mu_{\mathrm{p}}$ and function $\mathcal{S}$, and of the macroscopic deformation gradient $\overline{\mathbf{F}}$ and electric field $\overline{\mathbf{E}}$, in this case, through the electrostriction stretch $\bar{\lambda}$ and uniaxial component $\bar{E}$.

\subsubsection{The case of an isotropic distribution of monodisperse spherical particles}

For demonstration purposes and later use in Section 6, we spell out next the specialization of the general results (99) to the case discussed in subsection 4.2 when the microstructure consists of an isotropic distribution of monodisperse spherical particles that are either rigid or liquid-like.

Rigid particles. For the case of rigid spherical particles, the coefficients $\widetilde{\mu}_{0}, \widetilde{\varepsilon}_{0}, \widetilde{m}_{K_{0}}$ are given by expressions (85) so that relations (99) defining the electrostriction stretch $\bar{\lambda}$ and electric displacement $\bar{D}$ specialize to

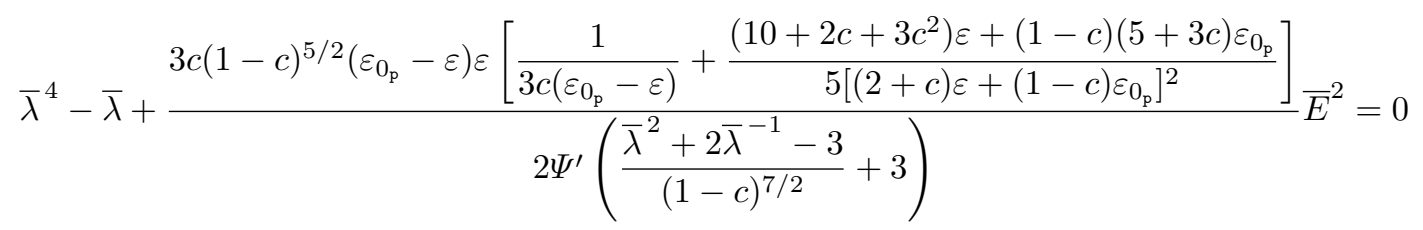

and

$$
\bar{D}=\varepsilon\left[\frac{3 c\left(\varepsilon_{0_{\mathrm{p}}}-\varepsilon\right)}{(2+c) \varepsilon+(1-c) \varepsilon_{0_{\mathrm{p}}}}\left(1+\frac{\left(1-\bar{\lambda}^{2}\right)\left[\left(10+2 c+3 c^{2}\right) \varepsilon+(1-c)(5+3 c) \varepsilon_{0_{\mathrm{p}}}\right]}{5\left[(2+c) \varepsilon+(1-c) \varepsilon_{0_{\mathrm{p}}}\right] \bar{\lambda}^{2}}\right)+\frac{1}{\bar{\lambda}^{2}}\right] \bar{E},
$$

where $\varepsilon_{0_{\mathrm{p}}}$ is solution of the nonlinear algebraic equation

$$
\mathcal{S}^{\prime}\left(\mathcal{I}_{5}^{\mathrm{Sph}_{r}}\right)-\frac{\varepsilon_{0_{\mathrm{p}}}}{2}=0
$$

with

$$
\mathcal{I}_{5}^{\mathrm{Sph}_{r}}=-\frac{54 c(1-c)\left(\varepsilon_{0_{\mathrm{p}}}-\varepsilon\right) \varepsilon^{2}}{5\left[(2+c) \varepsilon+(1-c) \varepsilon_{0_{\mathrm{p}}}\right]^{3}} \bar{E}^{2}+\frac{9\left[\left(10-c+6 c^{2}\right) \varepsilon+\left(5+c-6 c^{2}\right) \varepsilon_{0_{\mathrm{p}}}\right] \varepsilon^{2}}{5\left[(2+c) \varepsilon+(1-c) \varepsilon_{0_{\mathrm{p}}}\right]^{2}} \frac{\bar{E}^{2}}{\bar{\lambda}^{2}}
$$

If, in addition to being rigid, the particles are electrically conducting, the results (100)-(101) reduce to

$$
\bar{\lambda}^{4}-\bar{\lambda}+\frac{(1-c)^{3 / 2}\left(5+10 c+9 c^{2}\right) \varepsilon}{10 \Psi^{\prime}\left(\frac{\bar{\lambda}^{2}+2 \bar{\lambda}^{-1}-3}{(1-c)^{7 / 2}}+3\right)} \bar{E}^{2}=0
$$

and

$$
\bar{D}=\frac{\left[5+10 c+9 c^{2}\left(1-\bar{\lambda}^{2}\right)\right] \varepsilon}{5(1-c) \bar{\lambda}^{2}} \bar{E} .
$$

Liquid-like particles. For the case of liquid-like spherical particles, the coefficients $\widetilde{\mu}_{0}, \widetilde{\varepsilon}_{0}, \widetilde{m}_{K_{0}}$ are given by expressions (91) so that relations (99) defining the electrostriction stretch $\bar{\lambda}$ and electric displacement $\bar{D}$ take the form

$$
\bar{\lambda}^{4}-\bar{\lambda}+\frac{\varepsilon+\frac{9 \varepsilon\left(\varepsilon_{0_{\mathrm{p}}}-\varepsilon\right)^{2}}{\left[(2+c) \varepsilon+(1-c) \varepsilon_{0_{\mathrm{p}}}\right]^{2}}\left(\frac{4 c^{2}}{45}-\frac{81 c^{61 / 25}}{500}+\frac{c \varepsilon}{\varepsilon_{0_{\mathrm{p}}}-\varepsilon}\right)}{2(1-c)^{5 / 3} \Psi^{\prime}\left((1-c)^{2 / 3}\left[\bar{\lambda}^{2}+2 \bar{\lambda}^{-1}-3\right]+3\right)} \bar{E}^{2}=0
$$


and

$\bar{D}=\varepsilon\left[\frac{3 c\left(\varepsilon_{0_{\mathrm{p}}}-\varepsilon\right)}{(2+c) \varepsilon+(1-c) \varepsilon_{0_{\mathrm{p}}}}\left(1+\frac{\left(1-\bar{\lambda}^{2}\right)\left[\left(4500-400 c+729 c^{36 / 25}\right) \varepsilon+c\left(400-729 c^{11 / 25}\right) \varepsilon_{0_{\mathrm{p}}}\right]}{1500\left[(2+c) \varepsilon+(1-c) \varepsilon_{0_{\mathrm{p}}}\right] \bar{\lambda}^{2}}\right)+\frac{1}{\bar{\lambda}^{2}}\right] \bar{E}$

where $\varepsilon_{0_{\mathrm{p}}}$ is implicitly defined by the nonlinear algebraic equation

$$
\mathcal{S}^{\prime}\left(\mathcal{I}_{5}^{\mathrm{Sph}_{l}}\right)-\frac{\varepsilon_{0_{\mathrm{p}}}}{2}=0
$$

with

$\mathcal{I}_{5}^{\operatorname{Sph}_{l}}=\frac{3\left(1500-1900 c+729 c^{36 / 25}\right)\left(\varepsilon_{0_{\mathrm{p}}}-\varepsilon\right) \varepsilon^{2}}{250\left[(2+c) \varepsilon+(1-c) \varepsilon_{0_{\mathrm{p}}}\right]^{3}} \bar{E}^{2}-\frac{3\left[\left(750-1150 c+729 c^{36 / 25}\right)\left(\varepsilon_{0_{\mathrm{p}}}-\varepsilon\right)-2250 \varepsilon\right] \varepsilon^{2}}{250\left[(2+c) \varepsilon+(1-c) \varepsilon_{0_{\mathrm{p}}}\right]^{3}} \frac{\bar{E}^{2}}{\bar{\lambda}^{2}}$.

If, in addition to exhibiting a vanishingly small shear resistance, the particles are electrically conducting, the results (106)-(107) specialize further to

$$
\bar{\lambda}^{4}-\bar{\lambda}+\frac{\left(500-1000 c+900 c^{2}-729 c^{61 / 25}\right) \varepsilon}{1000(1-c)^{11 / 3} \Psi^{\prime}\left((1-c)^{2 / 3}\left[\bar{\lambda}^{2}+2 \bar{\lambda}^{-1}-3\right]+3\right)} \bar{E}^{2}=0
$$

and

$$
\bar{D}=\frac{\left[500-729 c^{61 / 25}\left(1-\bar{\lambda}^{2}\right)-500 c\left(2-3 \bar{\lambda}^{2}\right)+100 c^{2}\left(9-19 \bar{\lambda}^{2}\right)\right] \varepsilon}{500(1-c)^{2} \bar{\lambda}^{2}} \bar{E}
$$

\section{A hybrid FE formulation for homogenization problems in nonlinear electroelastostatics}

Complementary to the analytical framework put forth in the two preceding sections, we present in this section a hybrid FE formulation to construct homogenization solutions numerically for the macroscopic elastic dielectric response of dielectric elastomer composites subjected to finite deformations and finite electric fields. Non-hybrid FE formulations for this class of problems appear to have been first reported by $\mathrm{Li}$ and Landis (2012) and by Keip et al. (2014) in the context of two spatial dimensions, while non-hybrid FE formulations in three dimensions have been just recently reported by Miehe et al. (2016). These works also include sample solutions for square/cubic distributions of elliptical/ellipsoidal distributions of filler particles in compressible ideal elastic dielectric matrix materials under conditions of uniaxial electrostriction. Because of the well-known issue of volumetric locking, the above referenced non-hybrid formulations are not applicable to study incompressible or nearly incompressible dielectric elastomer composites, which are the case of most practical relevance and of interest here.

For definiteness, we restrict attention to dielectric elastomer composites of infinite extent whose initial microstructures are defined by the periodic repetition of a cubic unit cell, $Y=\left\{\mathbf{X}: \mathbf{X} \in[0,1]^{3}\right\}$ say, containing a finite, possibly large, number of filler particles. The hybrid FE formulation that we pursue here stems from the definitions of a function $\widehat{W}(\mathbf{X}, \mathbf{F}, J, \mathbf{E})$ that agrees identically with the local free-energy function $W(\mathbf{X}, \mathbf{F}, \mathbf{E})$ when $J=\operatorname{det} \mathbf{F}$ and of its transform (see, e.g., Chi et al., 2014)

$$
\widehat{W}^{*}(\mathbf{X}, \mathbf{F}, p, \mathbf{E}) \doteq \max _{J}\{p(J-1)-\widehat{W}(\mathbf{X}, \mathbf{F}, J, \mathbf{E})\}
$$

Provided that $\widehat{W}$ is convex in its argument $J$, the duality relation

$$
\widehat{W}(\mathbf{X}, \mathbf{F}, J, \mathbf{E})=\max _{p}\left\{p(J-1)-\widehat{W}^{*}(\mathbf{X}, \mathbf{F}, p, \mathbf{E})\right\}
$$


follows from (112). Direct use of (113) and restricting attention to $Y$-periodic solutions ${ }^{3}$ allows one to rewrite the variational problem (4) in the alternative form

$$
\bar{W}(\overline{\mathbf{F}}, \overline{\mathbf{E}}, c)=\min _{\mathbf{u} \in \mathcal{U}} \max _{\Phi \in \mathcal{F}} \max _{p \in \mathcal{P}} \int_{Y}\left\{p[\operatorname{det} \mathbf{F}(\mathbf{u})-1]-\widehat{W}^{*}(\mathbf{X}, \mathbf{F}(\mathbf{u}), p, \mathbf{E}(\Phi))\right\} \mathrm{d} \mathbf{X},
$$

where the displacement vector field $\mathbf{u}=\mathbf{x}-\mathbf{X}$ and the electric potential scalar field $\Phi$, both free of any differential constraint, are conveniently chosen as the independent arguments in lieu of the deformation gradient $\mathbf{F}$ and the curl-free Lagrangian electric field $\mathbf{E}$. For clarity, we write the dependence of $F_{i j}\left(u_{i}\right)=$ $\delta_{i j}+u_{i, j}$ and $E_{i}(\Phi)=-\Phi_{, i}$ on $\mathbf{u}$ and $\Phi$ explicitly. In the hybrid variational principle (114), $\mathcal{U}, \mathcal{F}$, and $\mathcal{P}$ stand for sufficiently large sets of admissible displacements $\mathbf{u}$, admissible electric potentials $\Phi$, and admissible pressure fields $p$ that are consistent with the following periodicity conditions:

$$
\begin{aligned}
& \mathbf{u}(\mathbf{X})=(\overline{\mathbf{F}}-\mathbf{I}) \mathbf{X}+\widetilde{\mathbf{u}}(\mathbf{X}), \text { where } \widetilde{\mathbf{u}} \text { is } Y \text {-periodic, } \int_{Y} \widetilde{\mathbf{u}}(\mathbf{X}) \mathrm{d} \mathbf{X}=\mathbf{0}, \quad \widetilde{\mathbf{u}}(\mathbf{0})=\mathbf{0}, \\
& \Phi(\mathbf{X})=-\overline{\mathbf{E}} \cdot \mathbf{X}+\widetilde{\Phi}(\mathbf{X}), \text { where } \widetilde{\Phi} \text { is } Y \text {-periodic, } \int_{Y} \widetilde{\Phi}(\mathbf{X}) \mathrm{d} \mathbf{X}=0, \quad \widetilde{\Phi}(\mathbf{0})=0, \\
& p \text { is } Y \text {-periodic. }
\end{aligned}
$$

The weak form of the Euler-Lagrange equations associated with the variational principle (114) reads as

$$
\begin{aligned}
& \int_{Y}\left\{p(\operatorname{det} \mathbf{F}(\mathbf{u})) F_{j i}^{-1}(\mathbf{u})-\frac{\partial \widehat{W}^{*}}{\partial F_{i j}}(\mathbf{X}, \mathbf{F}(\mathbf{u}), p, \mathbf{E}(\Phi))\right\} v_{i, j} \mathrm{~d} \mathbf{X}=0 \quad \forall \mathbf{v} \in \mathcal{U}^{0}, \\
& \int_{Y}\left\{\frac{\partial \widehat{W}^{*}}{\partial E_{i}}(\mathbf{X}, \mathbf{F}(\mathbf{u}), p, \mathbf{E}(\Phi))\right\} \psi_{, i} \mathrm{~d} \mathbf{X}=0 \quad \forall \psi \in \mathcal{F}^{0}, \\
& \int_{Y}\left\{\operatorname{det} \mathbf{F}(\mathbf{u})-1-\frac{\partial \widehat{W}^{*}}{\partial p}(\mathbf{X}, \mathbf{F}(\mathbf{u}), p, \mathbf{E}(\Phi))\right\} q \mathrm{~d} \mathbf{X}=0 \quad \forall q \in \mathcal{P},
\end{aligned}
$$

where $\mathcal{U}^{0}$ and $\mathcal{F}^{0}$ stand for sufficiently large spaces of vector fields $\mathbf{v}$ and scalar fields $\psi$ that are $Y$-periodic and satisfy $\mathbf{v}(\mathbf{0})=\mathbf{0}$ and $\psi(\mathbf{0})=0$.

A convenient yet robust method, which we pursue here, to construct numerical solutions for the type of Euler-Lagrange equations (116) is the conforming FE method. This requires the selection of suitable finite dimensional subspaces of $\mathcal{U}, \mathcal{F}$, and $\mathcal{P}$. The details of the construction of such subspaces and of the FE solutions for the Euler-Lagrange equations (116) that they allow to generate are provided in the Appendix.

\subsection{Application to non-Gaussian dielectric elastomers isotropically filled with nonlinear elastic dielectric particles}

The hybrid FE formulation presented in the Appendix is applicable to arbitrary types - in terms of local elastic dielectric behaviors as well as microstructures - of dielectric elastomer composites. The interest of this work is in non-Gaussian dielectric elastomers, characterized by free-energy functions of the form (10), isotropically filled with nonlinear elastic dielectric particles characterized by free-energy functions of the form (14). For this class of dielectric elastomer composites, the transform function $\widehat{W}^{*}(\mathbf{X}, \mathbf{F}, p, \mathbf{E})$ required in the hybrid variational principle (114) is simply given by

$$
\widehat{W}^{*}(\mathbf{X}, \mathbf{F}, p, \mathbf{E})=[1-\theta(\mathbf{X})] \widehat{W}_{\mathrm{m}}^{*}(\mathbf{F}, p, \mathbf{E})+\theta(\mathbf{X}) \widehat{W}_{\mathrm{p}}^{*}(\mathbf{F}, p, \mathbf{E})
$$

with

\footnotetext{
${ }^{3}$ Periodic solutions of larger period than the unit cell $Y$ may exist. These are associated with the development of microscopic instabilities (see, e.g., Geymonat et al., 1993; Michel et al., 2010). We shall not consider such solutions in this work.
} 


$$
\widehat{W}_{\mathrm{m}}^{*}(\mathbf{F}, p, \mathbf{E})=-\Psi\left(I_{1}\right)+\frac{\varepsilon}{2} I_{5}^{E} \quad \text { and } \quad \widehat{W}_{\mathrm{p}}^{*}(\mathbf{F}, p, \mathbf{E})=-\frac{\mu_{\mathrm{p}}}{2}\left[I_{1}-3\right]+\mathcal{S}\left(I_{5}^{E}\right),
$$

this for any isotropic indicator function $\theta$ of choice.

Now, by definition, an isotropic distribution of particles involves an infinite number of particles. Within the present context, however, it suffices to consider a large but finite number of particles randomly distributed in the repeating unit cell $Y$. In this paper, we follow this approach to work out FE results for two types of microstructures: $i$ ) an isotropic distribution of polydisperse spherical particles of a finite number of different sizes and ii) an isotropic distribution of monodisperse spherical particles, as described in Sections 4.1 and 4.2 of Part I. Representative meshes for two realizations of such microstructures are shown in Fig. 2 for a composite where the volume fraction of particles is $c=0.15$. Mesh sensitivity analyses have indicated that meshes comprising $N_{e} \approx 150,000$ elements ( $N_{n} \approx 670,000$ nodes) for the polydisperse microstructure and $N_{e} \approx 80,000$ elements $\left(N_{n} \approx 345,000\right.$ nodes) for the monodisperse microstructure are refined enough to deliver accurate results, at least for the range of volume fraction of particles considered in this work, $c \in[0,0.25]$. Finally, we note that all the FE results presented throughout this work correspond to the average of three realizations (see Sections 4.1 and 4.2 of Part I). The responses of all three realizations exhibited very small differences (less than $1 \%$ ) between one another.

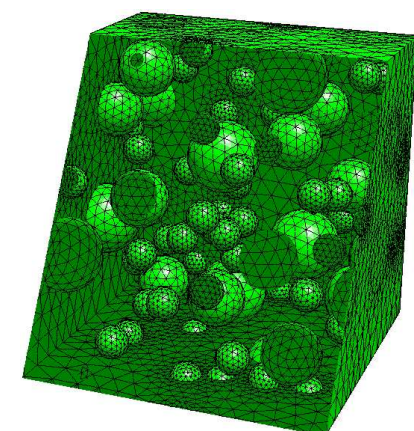

(a)

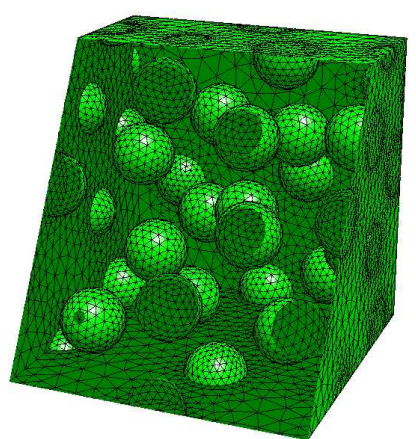

(b)

Figure 2: Meshes for unit cells $Y$ containing: (a) $N=36$ randomly distributed spherical particles of three different sizes with $N_{e} \approx 150,000$ elements $\left(N_{n} \approx 670,000\right.$ nodes) and (b) $N=30$ randomly distributed spherical particles of identical size with $N_{e} \approx 80,000$ elements $\left(N_{n} \approx 345,000\right.$ nodes). The volume fraction of particles in both meshes is $c=0.15$.

\section{Sample results}

Next, we present sample results for the macroscopic electromechanical behaviors of a typical nonGaussian dielectric elastomer filled with several physically significant types of nonlinear elastic dielectric filler particles, as characterized by the analytical variational solution (56) and by the hybrid FE formulation of Section 5. Throughout this section, for definiteness, we make use of the choice (12) for the function $\Psi\left(I_{1}\right)$ described in subsection 2.1 , so that

$$
W_{\mathrm{m}}(\mathbf{F}, \mathbf{E})=\left\{\begin{array}{ll}
\frac{3^{1-\alpha_{1}}}{2 \alpha_{1}} \mu_{1}\left[I_{1}^{\alpha_{1}}-3^{\alpha_{1}}\right]+\frac{3^{1-\alpha_{2}}}{2 \alpha_{2}} \mu_{2}\left[I_{1}^{\alpha_{2}}-3^{\alpha_{2}}\right]-\frac{\varepsilon}{2} I_{5}^{E} & \text { if } \quad \operatorname{det} \mathbf{F}=1 \\
+\infty & \text { otherwise }
\end{array},\right.
$$

with the material parameters listed in Table 1. This choice of free-energy function provides an accurate model for a standard silicone elastomer over large ranges of deformations and electric fields; see Section 2.3 in Lopez-Pamies (2010) and Liu et al. (2013).

\begin{tabular}{|l|c|c|c|c|c|}
\cline { 2 - 6 } \multicolumn{1}{c|}{} & $\alpha_{1}$ & $\mu_{1}(\mathrm{MPa})$ & $\alpha_{2}$ & $\mu_{2}(\mathrm{MPa})$ & $\varepsilon(\mathrm{F} / \mathrm{m})$ \\
\hline Silicone elastomer & 3.837 & 0.032 & 0.559 & 0.3 & $3.2 \varepsilon_{0}$ \\
\hline
\end{tabular}

Table 1: Values for the materials parameters $\alpha_{1}, \mu_{1}, \alpha_{2}, \mu_{2}, \varepsilon$ in the free-energy function (119) fitted to model a silicone elastomer. 
Throughout this section, we also make use of the choice (19) for the function $\mathcal{S}\left(I_{5}^{E}\right)$, so that the free-energy function characterizing the response of the particles is given by

$$
W_{\mathrm{p}}(\mathbf{F}, \mathbf{E})= \begin{cases}\frac{\mu_{\mathrm{p}}}{2}\left[I_{1}-3\right]-\frac{\varepsilon_{0}}{2} I_{5}^{E}- & p_{s}^{2} \\ \frac{3\left(\varepsilon_{\mathrm{p}}-\varepsilon_{0}\right)}{+\infty}\left[\ln \left(\sinh \left(\frac{3\left(\varepsilon_{\mathrm{p}}-\varepsilon_{0}\right) \sqrt{I_{5}^{E}}}{p_{s}}\right)\right)-\ln \left(\frac{3\left(\varepsilon_{\mathrm{p}}-\varepsilon_{0}\right) \sqrt{I_{5}^{E}}}{p_{s}}\right)\right] & \text { if } \quad \operatorname{det} \mathbf{F}=1 \\ \text { otherwise }\end{cases}
$$

In particular, within the context of this model, we consider the sets of initial shear modulus $\mu_{\mathrm{p}}$, initial permittivity $\varepsilon_{\mathrm{p}}$, and saturation polarization $p_{s}$ that are listed in Table 2 . These correspond to the practically relevant cases of rigid conducting particles, rigid high-permittivity particles exhibiting polarization saturation, and liquid-like high-permittivity particles. They are representative of filler particles made out of metals or hard conducting polymers, polarization-saturating ferroelectric ceramics, and high-permittivity liquids, the first two of which have been widely utilized in experiments (see, e.g., Zhang et al., 2002; Huang et al., 2005; McCarthy et al., 2009; Liu et al., 2013).

\begin{tabular}{|l|c|c|c|}
\cline { 2 - 4 } \multicolumn{1}{c|}{} & $\mu_{\mathrm{p}}(\mathrm{MPa})$ & $\varepsilon_{\mathrm{p}}(\mathrm{F} / \mathrm{m})$ & $p_{s}\left(\mathrm{C} / \mathrm{m}^{2}\right)$ \\
\hline Rigid conducting particles & $+\infty$ & $+\infty$ & $+\infty$ \\
\hline $\begin{array}{l}\text { Rigid high-permittivity particles } \\
\text { with polarization saturation }\end{array}$ & $+\infty$ & $320 \varepsilon_{0}$ & $10^{-2}, 10^{-4}$ \\
\hline $\begin{array}{l}\text { Liquid-like high-permittivity } \\
\text { particles }\end{array}$ & 0.03 & $320 \varepsilon_{0}$ & $+\infty$ \\
\hline
\end{tabular}

Table 2: Values for the materials parameters $\mu_{\mathrm{p}}, \varepsilon_{\mathrm{p}}, p_{s}$ in the free-energy function (120) utilized to model rigid conducting particles, rigid high-permittivity particles with polarization saturation, and liquid-like high-permittivity particles.

We begin in subsection 6.1 by reporting sample results that assess the accuracy of the variational solution (56) via direct comparisons with FE results. For conciseness, we restrict attention to microstructures wherein the fillers are monodisperse spherical particles. The analytical solution (56) is subsequently deployed to probe the electrostriction response of the above-introduced non-Gaussian silicone elastomer filled with rigid conducting particles (subsection 6.2), rigid high-permittivity particles with polarization saturation (subsection 6.3), and liquid-like high-permittivity particles (subsection 6.4). These latter sets of results are aimed at scrutinizing the effects of the elastic dielectric properties of the underlying fillers, as well as their content and size dispersion on the macroscopic electrostriction properties of promising classes of dielectric elastomer composites.

\subsection{Accuracy of the variational solution (56) at finite deformations and finite electric fields}

Figure 3 presents comparisons between the variational solution (56) and corresponding FE solutions. As for all the results presented in this section, the underlying dielectric elastomeric matrix corresponds to the non-Gaussian silicone elastomer characterized by the free-energy function (119) with material parameters given in Table 1. The fillers are monodisperse spherical particles whose elastic dielectric behaviors are characterized by the free-energy function (120) with material parameters given in Table 2. Specifically, Figs. 3(a) and (b) present comparisons for the case of rigid conducting particles with volume fraction $c=0.05$. Figures $3(\mathrm{c})$ and $(\mathrm{d})$ show comparisons for rigid high-permittivity particles with polarization saturation $p_{s}=10^{-4} \mathrm{C} / \mathrm{m}^{2}$ at volume fraction $c=0.05$. Figures $3(\mathrm{e}$ ) and (f) pertain to liquid-like high-permittivity particles with volume fraction $c=0.15$.

The plots in Fig. 3 show the effective free energy $\bar{W}$ as a function of the invariants $\bar{I}_{2}$ and $\bar{I}_{6}^{E}$ for fixed values of the remaining four isotropic invariants, $\bar{I}_{1}, \bar{I}_{4}^{E}, \bar{I}_{5}^{E}, \bar{I}_{6}^{E}$ and $\bar{I}_{1}, \bar{I}_{2}, \bar{I}_{4}^{E}, \bar{I}_{5}^{E}$. We note that keeping the values of four out of the five invariants $\bar{I}_{1}, \bar{I}_{2}, \bar{I}_{4}^{E}, \bar{I}_{5}^{E}, \bar{I}_{6}^{E}$ constant restricts the range of physical values that the remaining free invariant can take on. For instance, for the prescribed set of invariants $\bar{I}_{1}=3.86, \bar{I}_{4}^{E}=7.1 \times 10^{3} \mathrm{MV}^{2} / \mathrm{m}^{2}, \bar{I}_{5}^{E}=11.7 \times 10^{3} \mathrm{MV}^{2} / \mathrm{m}^{2}, \bar{I}_{6}^{E}=30.0 \times 10^{3} \mathrm{MV}^{2} / \mathrm{m}^{2}$ in Fig. 3(a), 
the range of physically allowable values for $\bar{I}_{2}$ is $[4.13,4.24]$. The results shown in Fig. 3 span the entire range of physically allowable values for each one of the cases that is presented. We further note that the selected values of the macroscopic invariants $\bar{I}_{1}, \bar{I}_{2}, \bar{I}_{4}^{E}, \bar{I}_{5}^{E}, \bar{I}_{6}^{E}$ in Fig. 3 involve local invariants $I_{1}(\mathbf{X})$ and $I_{5}^{E}(\mathbf{X})$ that are large enough in the silicone elastomer and in the filler particles so as to trigger the non-Gaussian stiffening of the former and, in the pertinent case, the polarization saturation of the latter. As an example to visually illustrate this point, Figs. 4 (a) and (b) show contour plots of the local invariants $I_{1}(\mathbf{X})$ and $I_{5}^{E}(\mathbf{X})$ from one of the FE solutions for the case of rigid high-permittivity particles with polarization saturation $p_{s}=10^{-4} \mathrm{C} / \mathrm{m}^{2}$ at volume fraction $c=0.05$.

An immediate observation from Fig. 3 is that the variational solution (56) is in good quantitative agreement with corresponding FE solutions for all three types of filler particles considered. The variational solution (56) is also seen to be in good qualitative agreement with the FE results in that it is independent of the invariants $\bar{I}_{2}$ and $\bar{I}_{6}^{E}$. This remarkable functional trait had already been observed in the simpler uncoupled context of the overall nonlinear elastic response of non-Gaussian rubber isotropically filled with rigid particles (Lopez-Pamies et al., 2013), which corresponds to setting $\overline{\mathbf{E}}=\mathbf{0}$ and $\mu_{\mathrm{p}}=+\infty$ in the present context. A large body of results (in addition to those presented here) has confirmed that the variational solution (56) remains in good qualitative and quantitative agreement with "exact" numerical 


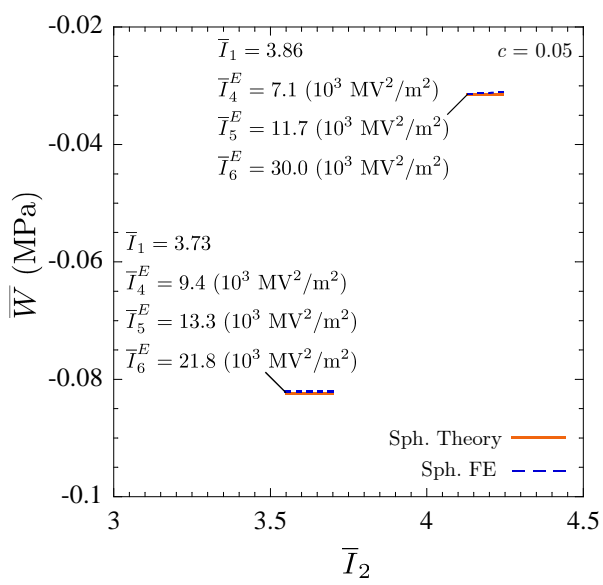

(a)

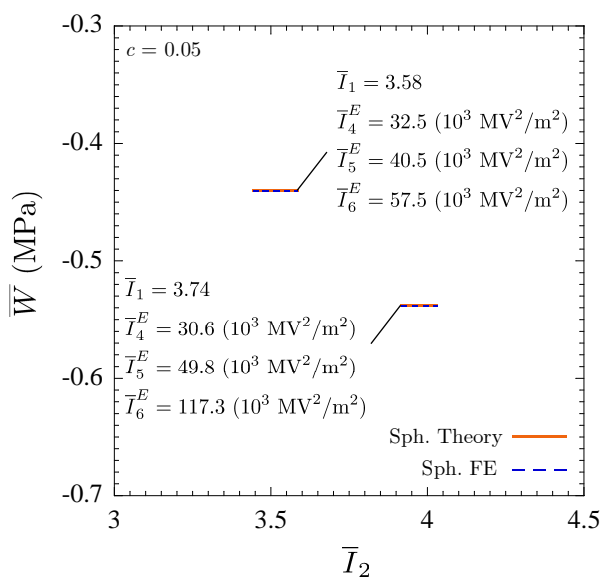

(c)

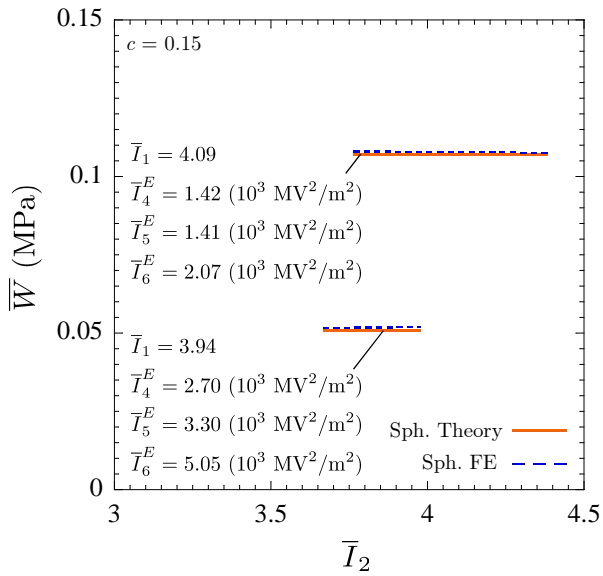

(e)

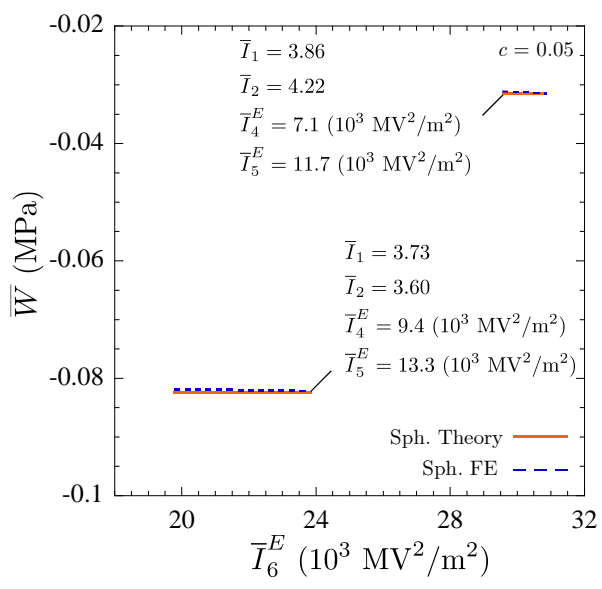

(b)

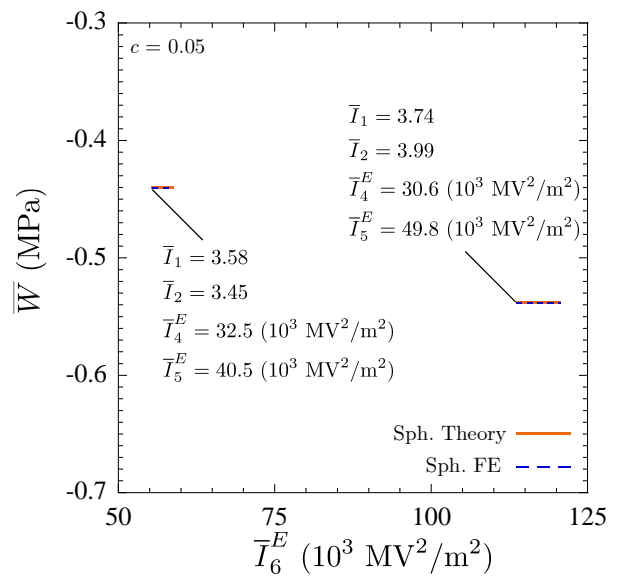

(d)

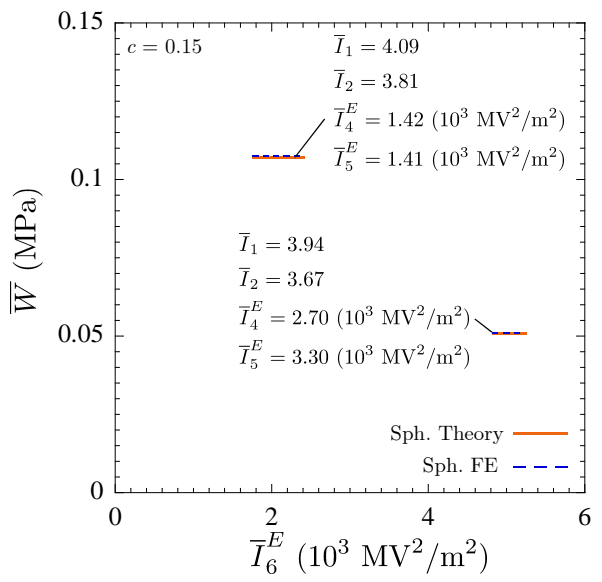

(f)

Figure 3: Plots of the effective free energy $\bar{W}$ for a non-Gaussian silicone elastomer, characterized by the free-energy function (119) with the material parameters listed in Table 1, filled with: (a)-(b) a volume fraction $c=0.05$ of rigid conducting spherical particles, (c)-(d) a volume fraction $c=0.05$ of rigid high-permittivity spherical particles with polarization saturation $p_{s}=10^{-4} \mathrm{C} / \mathrm{m}^{2}$, and (e)-(f) a volume fraction $c=0.15$ of liquid-like high-permittivity spherical particles. Results are shown for the values of the free energy in terms of the invariants $\bar{I}_{2}$ and $\bar{I}_{6}^{E}$ for two sets of fixed values of the remaining four isotropic invariants. The solid lines (labeled "Sph. Theory") correspond to appropriate specializations of the variational solution (56), namely, expressions (90), (87), and (93) for rigid conducting, rigid with polarization-saturating, and liquid-like high-permittivity monodisperse spherical particles, respectively. The dashed lines (labeled "Sph. FE") correspond to the FE solutions. 
solutions for finite deformations and finite electric fields irrespectively of the elastic dielectric properties of the dielectric elastomer and filler particles, as characterized by $\Psi\left(I_{1}\right), \varepsilon, \mu_{p}, \mathcal{S}\left(I_{5}^{E}\right)$, and irrespectively of the specifics of the underlying microstructure, at least for volume fractions of particles sufficiently away from percolation.

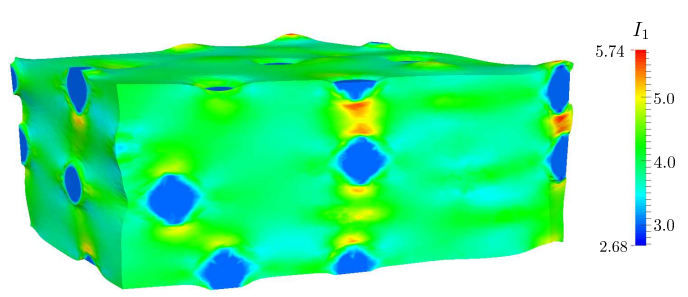

(a)

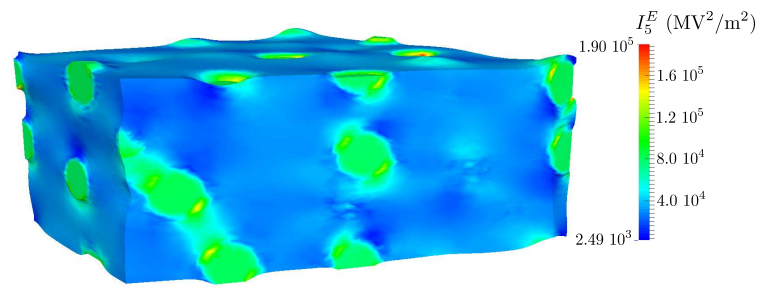

(b)

Figure 4: Representative contour plots of the local invariants $I_{1}(\mathbf{X})$ and $I_{5}^{E}(\mathbf{X})$ in a non-Gaussian silicone elastomer, characterized by the free energy function (119) with the material parameters listed in Table 1, filled with a volume fraction $c=0.05$ of rigid high-permittivity spherical particles with polarization saturation. The plots correspond to values of the macroscopic invariants $\bar{I}_{1}=3.74$ and $\bar{I}_{5}^{E}=49.8 \times 10^{3} \mathrm{MV}^{2} / \mathrm{m}^{2}$.

\subsection{Rigid conducting particles}

Figure 5 presents results determined from the variational solution (56) for the macroscopic response of the non-Gaussian silicone elastomer, again, characterized by the free-energy function (119) with the material parameters listed in Table 1, filled with rigid conducting particles under the conditions of electrostriction (97). Parts (a) and (c) of the figure pertain to results (labeled "HJ Theory") for the iterative microstructure described in subsection 4.1, wherein the particles are infinitely polydisperse in size. On the other hand, parts (b) and (d) correspond to the case of monodisperse spherical particles (labeled "Sph. Theory") described in subsection 4.2. Parts (e) and (f) include results for both microstructures. To further illustrate the accuracy of the variational solution (56), the FE solutions for the microstructure with spherical particles are also included in the appropriate parts of the figure (up to the point at which we were able to compute them). These solutions are labeled "Sph. FE" and are displayed as dashed lines or solid circles. The response of the unfilled silicone elastomer (dotted line) is also displayed in the figures for comparison purposes.

The plots in Figs. 5(a) through (d) show results for the electrostriction stretch $\bar{\lambda}$ in terms of the applied electric field $\bar{E}$, as defined by equation $(99)_{1}$, for two volume fractions of particles, $c=0.05$ and $c=0.15$. A plain observation from these four sets of plots is that, irrespectively of the microstructure, the addition of rigid conducting particles has little effect on the electrostriction response of the silicone elastomer, at least up to around the stretch $\bar{\lambda} \approx 0.62$ corresponding to the point at which the electric field $\bar{E}$ reaches a local maximum, $\bar{E} \approx 74.2 \mathrm{MV} / \mathrm{m}$, in the unfilled silicone elastomer. Indeed, up to that point, the dielectric elastomer composite with the iterative microstructure deforms only slightly less than the unfilled silicone elastomer for both volume fractions of particles considered, while the dielectric elastomer composite with spherical particles deforms only slightly more. For sufficiently large electric fields $\bar{E}>74.2 \mathrm{MV} / \mathrm{m}$, the presence of rigid conducting particles does consistently lead to a sizable reduction in the electrostriction when compared to that of the unfilled silicone elastomer. In particular, larger volume fractions $c$ of particles lead to larger reductions. These results are in striking disagreement with most experimental investigations, which have reported enhancements in electrostriction up to several thousands of a percent for small additions $(c<0.1)$ of stiff conducting particles (see, e.g., Huang et al., 2005). The implications of this disagreement are discussed in Section 7. 


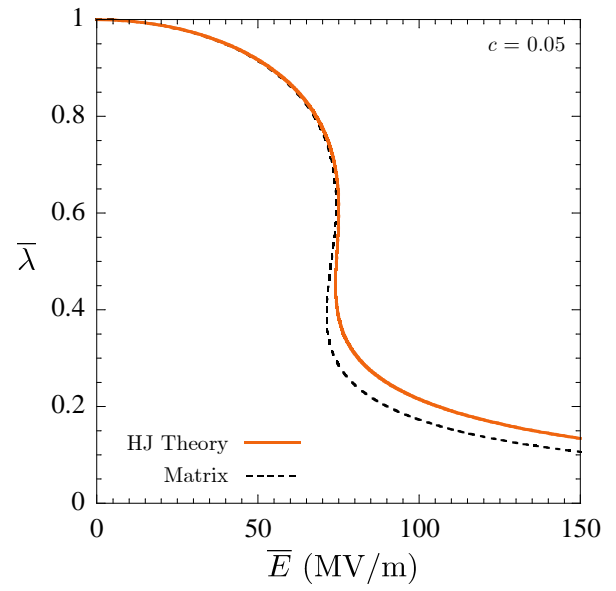

(a)

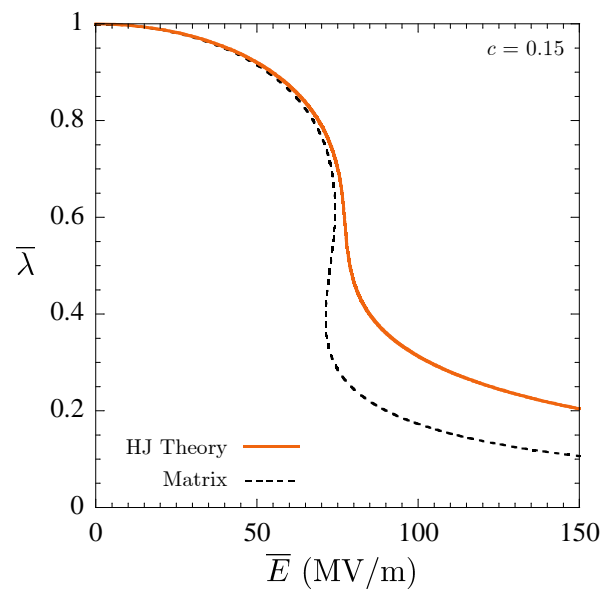

(c)

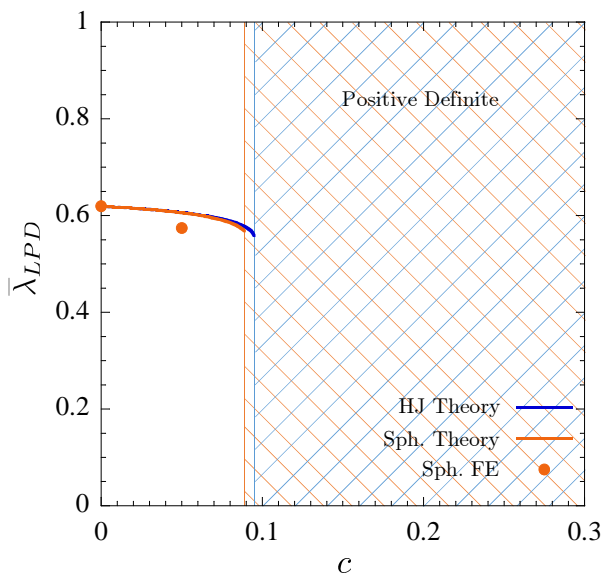

(e)

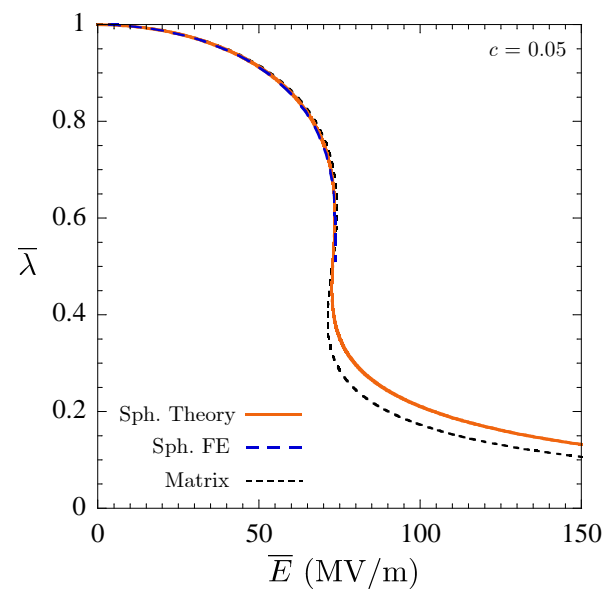

(b)

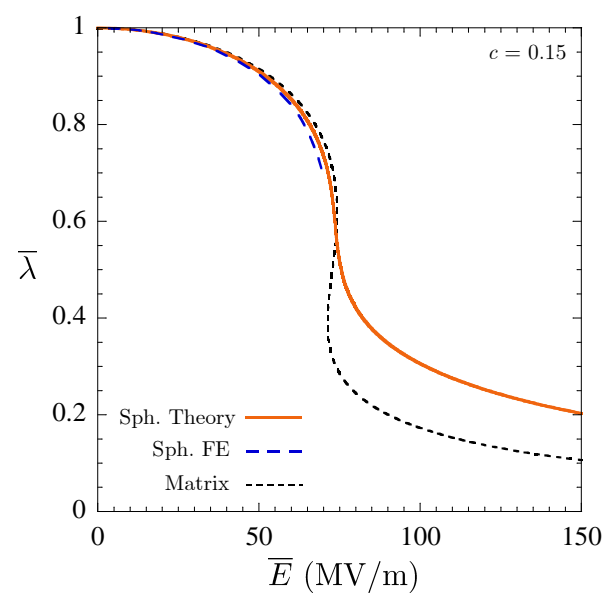

(d)

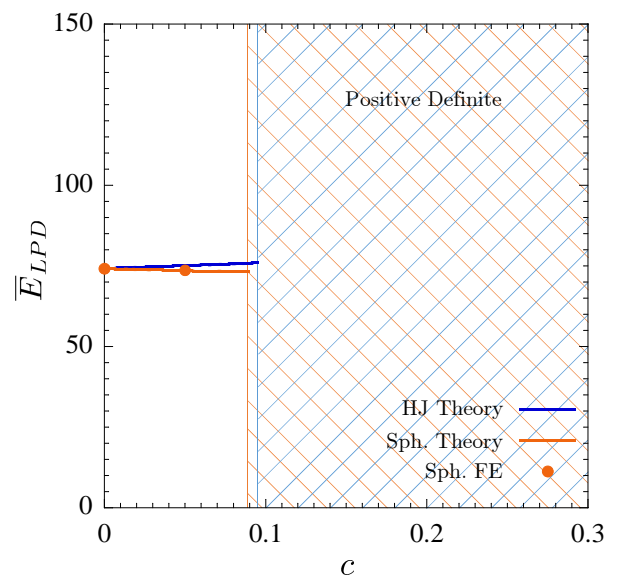

(f)

Figure 5: Electrostriction response determined from the effective free energy (56) under conditions (97) - labeled "Theory" and displayed as solid lines in the plots - for a non-Gaussian silicone elastomer, characterized by the free energy function (119) with the material parameters listed in Table 1, filled with rigid conducting particles at volume fraction $c$. Results are shown for the infinitely polydisperse iterative microstructure (labeled "HJ") and for the microstructure with monodisperse spherical particles (labeled "Sph."). To further illustrate the accuracy of the variational solution (56), corresponding plots are also included of the FE solutions for the microstructure with spherical particles (labeled "FE" and displayed as dashed lines or solid circles). 
Another salient observation from Figs. 5(a) through (d) is that, much like for the unfilled silicone elastomer, the applied electric field $\bar{E}$ for the dielectric elastomer composites with volume fraction of particles $c=0.05$ - but not for those with the larger volume fraction $c=0.15$ - reaches a local maximum, $\bar{E}_{L P D}$ say, at some critical electrostriction stretch, $\bar{\lambda}_{L P D}$ say. As mentioned in remark viii above in Section 4 and discussed in detail in Section 5.2 of Part I, this point corresponds to the loss of positive definiteness of the tangent modulus of the effective Helmholtz free energy (52). The values of the critical electrostriction stretch $\bar{\lambda}_{L P D}$ and corresponding critical electric field $\bar{E}_{L P D}$ are plotted in Figs. $5(\mathrm{e})$ and (f) for both microstructures as functions of the volume fraction $c$ of particles. Remarkably, these plots indicate that the addition of rigid conducting particles has little effect on $\bar{\lambda}_{L P D}$ and $\bar{E}_{L P D}$ up to some threshold in $c$ beyond which any further addition of particles results in stable dielectric elastomers composites that remain positive definite for arbitrarily large electric fields $\bar{E}$, in spite of the fact, again, that the underlying silicone matrix loses positive definiteness at $\bar{\lambda} \approx 0.62$ and $\bar{E}_{L P D} \approx 74.2 \mathrm{MV} / \mathrm{m}$. The threshold for the iterative microstructure with infinitely polydisperse particles is given approximately by $c=0.095$, while that for the microstructure with monodisperse spherical particles is given approximately by $c=0.089$.

\subsection{Rigid high-permittivity particles with polarization saturation}

Figures 6(a) through (d) show results analogous to those shown in Figs. 5(a) through (d) for the case of rigid high-permittivity particles with polarization saturation, whose behaviors are characterized by the free-energy function (120) with the material parameters indicated in Table 2. A quick glance at the plots suffices to recognize that the addition of particles with the larger polarization saturation $p_{s}=10^{-2} \mathrm{C} / \mathrm{m}^{2}$ leads to electrostriction responses that are not much different from the responses of the above-discussed dielectric elastomer composites wherein the particles are electrically conducting, especially for the case of volume fraction of particles $c=0.05$. On the other hand, the addition of particles with the smaller polarization saturation $p_{s}=10^{-4} \mathrm{C} / \mathrm{m}^{2}$ consistently leads to a drastic reduction in the electrostriction response for all values of the applied electric field $\bar{E}$ when compared to the response of the unfilled silicone elastomer. These results are, again, in disagreement with a number of experimental investigations, which have reported significant enhancements in the electrostriction response of dielectric elastomers when filled with small amounts $(c<0.1)$ of stiff polarization-saturating particles (see, e.g., Liu et al., 2013). The implications of this disagreement too are discussed in Section 7.

To gain further insight into the effects of the polarization saturation properties of the filler particles, Figs. 6(e) and (f) present results, as functions of $p_{s}$, for the critical electrostriction stretch $\bar{\lambda}_{L P D}$ and associated critical electric field $\bar{E}_{L P D}$ at which the dielectric elastomer composites with polarizationsaturating particles reach an electromechanical limit load. Results are shown for both microstructures, with infinitely polydisperse particles ("HJ") and with monodisperse spherical particles ("Sph."), for a volume fraction of particles $c=0.05$. It is evident from the plots that the electromechanical limit load in these classes of dielectric elastomer composites is largely unaffected by the polarization saturation of the particles.

A final point worth remarking from all six sets of plots included in Fig. 6 is that both microstructures ("HJ" and "Sph.") exhibit nearly identical behaviors. This suggests that the response of non-Gaussian dielectric elastomers isotropically filled with rigid high-permittivity particles with polarization saturation is fairly insensitive, sufficiently away from percolation, to fine microstructural details (such as the size dispersion and the shape of the filler particles) beyond the volume fraction of particles, even more so than the non-Gaussian dielectric elastomers isotropically filled with rigid conducting particles discussed in the preceding subsection. 


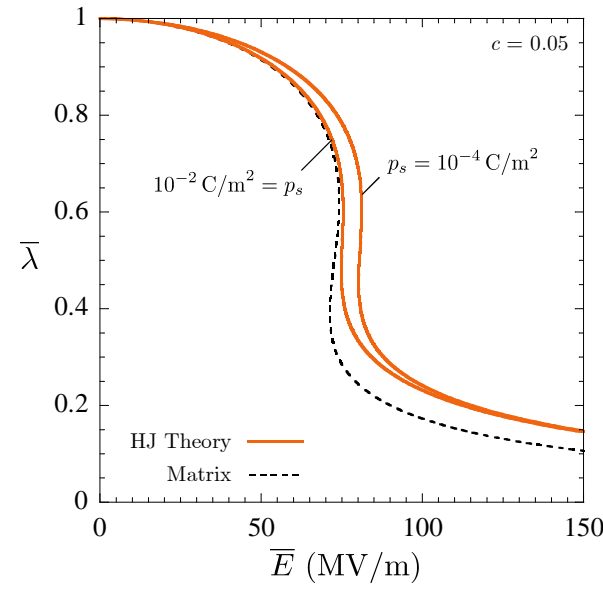

(a)

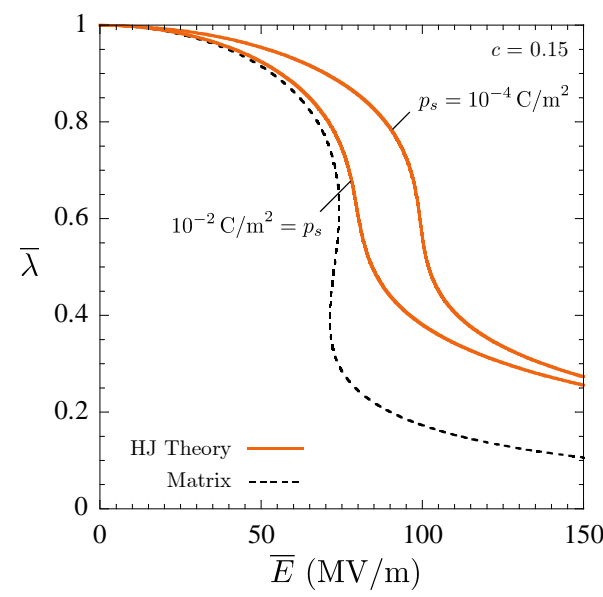

(c)

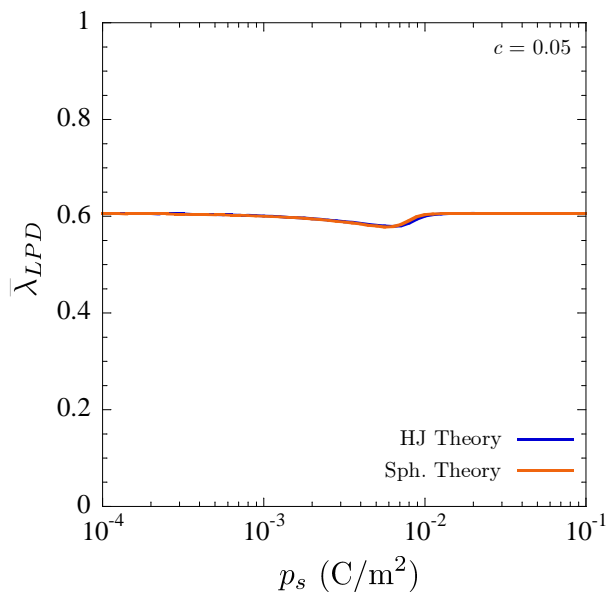

(e)

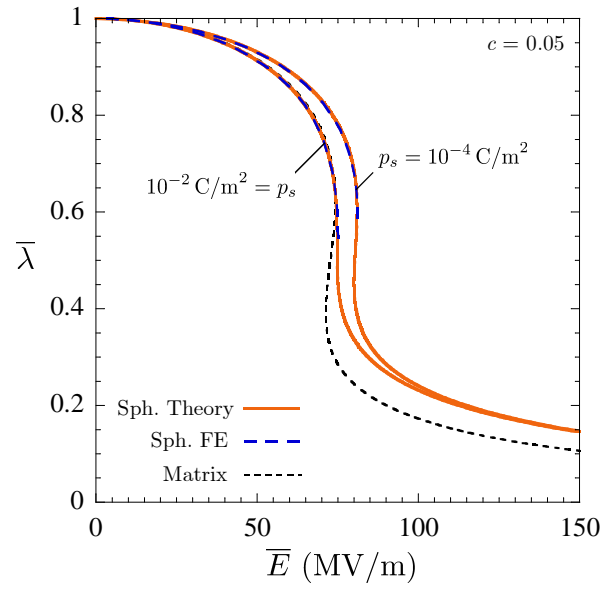

(b)

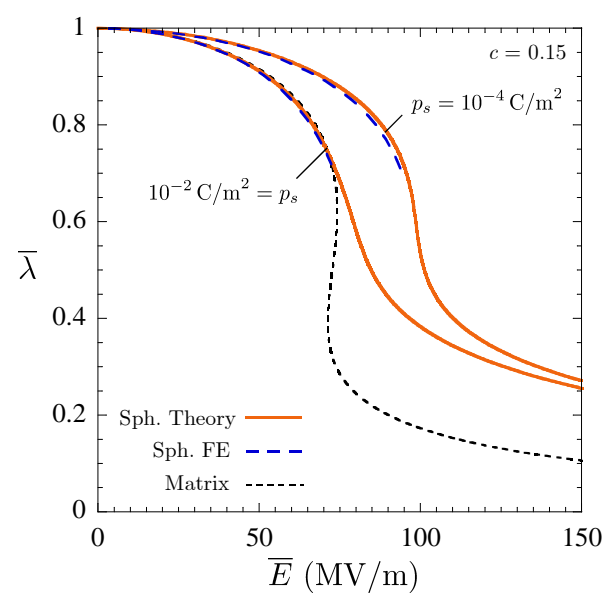

(d)

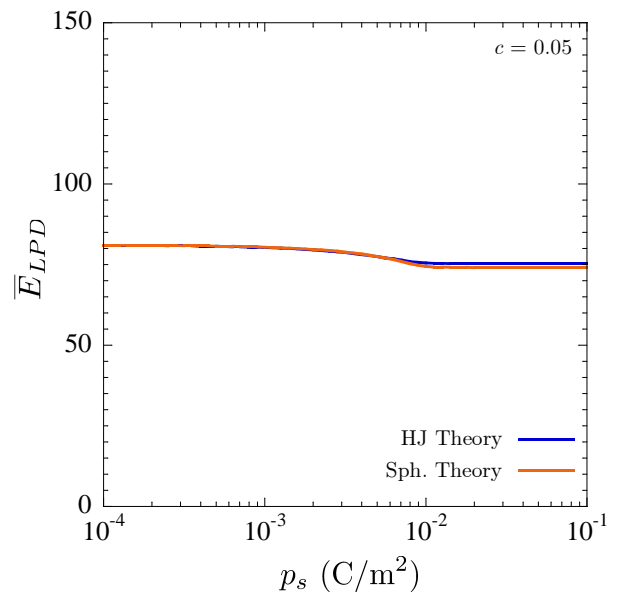

(f)

Figure 6: Electrostriction response determined from the effective free energy (56) under conditions (97) — labeled "Theory" and displayed as solid lines in the plots - for a non-Gaussian silicone elastomer, characterized by the free energy function (119) with the material parameters listed in Table 1, filled with rigid high-permittivity particles with polarization saturation at volume fraction $c$. The behavior of the particles is characterized by the free-energy function (120) with the material parameters indicated in Table 2. Results are shown for the infinitely polydisperse iterative microstructure (labeled "HJ") and for the microstructure with monodisperse spherical particles (labeled "Sph."). Corresponding FE solutions are also included for the microstructure with spherical particles (labeled "FE" and displayed as dashed lines). 


\subsection{Liquid-like high-permittivity particles}

Finally, we consider the electrostriction response of the non-Gaussian silicone elastomer when filled with liquid-like high-permittivity particles, whose behaviors are characterized by the free-energy function (120) with the material parameters indicated in Table 2. Results analogous to those presented in Fig. 5 are shown in Fig. 7. As opposed to the addition of rigid conducting particles and of rigid high-permittivity particles with polarization saturation, the addition of liquid-like high-permittivity particles is seen to lead to significant enhancements in electrostriction. In particular, larger volume fractions of particles consistently lead to larger enhancements. This is accompanied, however, by a sizable monotonic decrease in the limiting electric field $\bar{E}_{L P D}$ while the corresponding stretch $\bar{\lambda}_{L P D}$ remains fairly constant. The authors are not aware of any experimental investigation on dielectric elastomers isotropically filled with liquid-like high-permittivity particles. The results presented in Fig. 7 certainly motivate their pursuit.

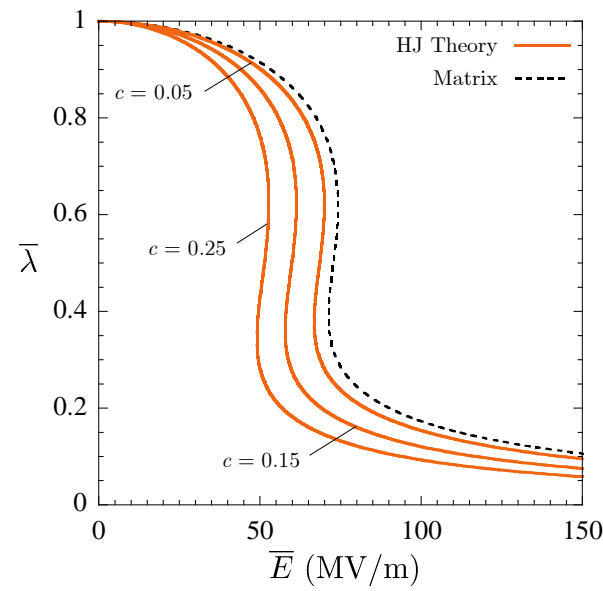

(a)

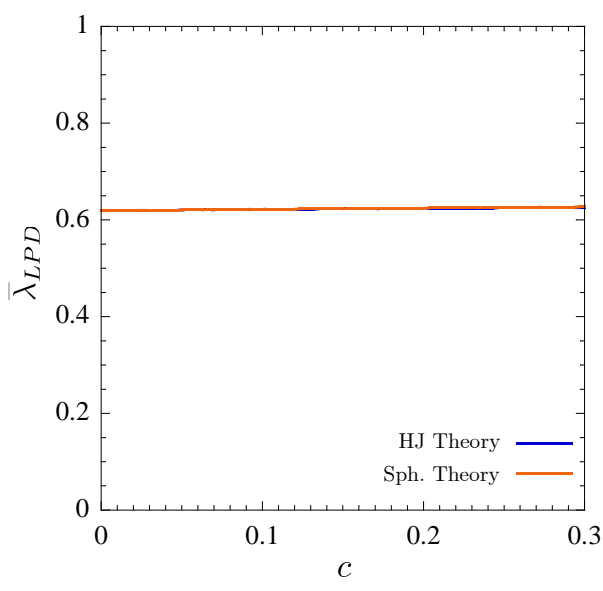

(c)

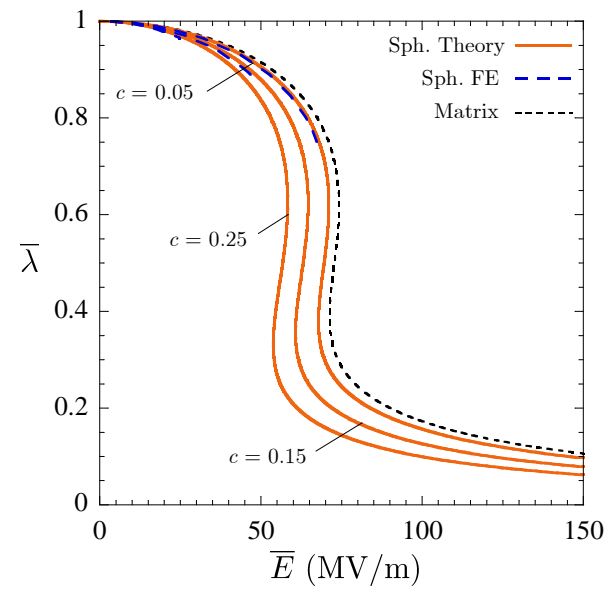

(b)

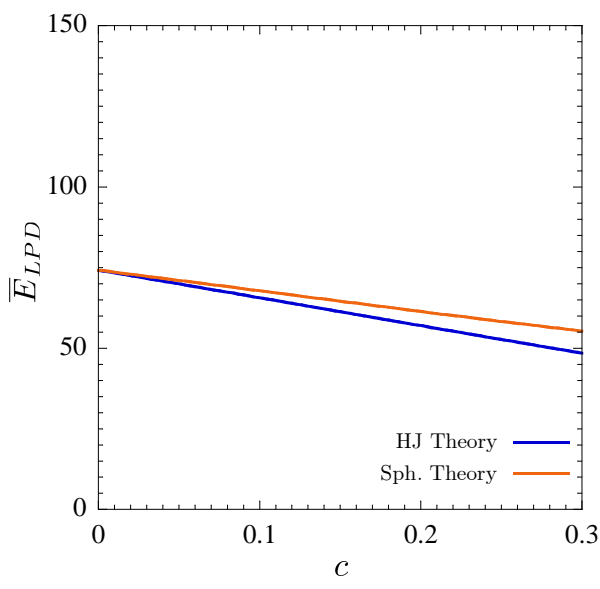

(d)

Figure 7: Electrostriction response determined from the effective free energy (56) under conditions (97) - labeled "Theory" and displayed as solid lines in the plots - for a non-Gaussian silicone elastomer, characterized by the free energy function (119) with the material parameters listed in Table 1, filled with liquid-like high-permittivity particles at volume fraction $c$. The behavior of the particles is characterized by the free-energy function (120) with the material parameters indicated in Table 2. Results are shown for the infinitely polydisperse iterative microstructure (labeled "HJ") and for the microstructure with monodisperse spherical particles (labeled "Sph."). Corresponding FE solutions are also included for the microstructure with spherical particles (labeled "FE" and displayed as dashed lines).

We conclude by remarking that similar to the two previous cases involving rigid filler particles, all four sets of plots displayed in Fig. 7 show that both microstructures ("HJ" and "Sph.") exhibit nearly identical behaviors. This suggests that the response of non-Gaussian dielectric elastomers isotropically filled with liquid-like particles too is largely insensitive to fine microstructural details beyond the volume fraction of 
particles (again, sufficiently away from percolation). Further evidence supporting this lack of sensitivity is provided by the fact that, up to the volume fraction of particles considered in this work $c=0.25$, the FE solutions for the isotropic distribution of polydisperse spherical particles with three different sizes described in Section 5 are virtually indistinguishable from those presented in Figs. 5 through 7 for monodisperse particles.

\section{Comparisons with experimental data and final comments}

Since the "rediscovery" of electroactive polymers at around the turn of the millennium, numerous experimental investigations have reported that dielectric elastomers filled with small amounts of (semi)conducting or high-permittivity particles exhibit electrostriction properties that are far superior to those of the corresponding unfilled dielectric elastomers (see, e.g., Zhang et al., 2002; Huang and Zhang, 2004; Huang et al., 2005; Carpi and Rossi, 2005; McCarthy et al., 2009, Meddeb and Ouanies, 2012; Liu et al., 2013). Motivated by a heuristic analysis in the asymptotic context of small deformations and moderate electric fields, $\mathrm{Li}$ (2003) and $\mathrm{Li}$ et al. (2004) conjectured that such superior properties are due to the nonlinear elastic dielectric nature of elastomers which heightens the role of the fluctuations of the electric field in the presence of filler particles. Still within the asymptotic context of small deformations and moderate electric fields, Tian et al. (2012) extended and made rigorous the approximate analysis of Li (2003) and Li et al. (2004) and also pointed to the nonlinear elastic dielectric nature of elastomers as the dominant electrostriction enhancing mechanism. Armed with the general analytical solution (56) and the hybrid FE formulation presented in this work, we are now in a position to probe the verity of this initial conjecture. Contrary to it, the sample results for finite deformations and finite electric fields presented in subsections 6.2 and 6.3 have already suggested that the nonlinear elastic dielectric nature of elastomers is not the mechanism responsible for the enhanced electrostriction properties exhibited by emerging dielectric elastomer composites. In the sequel, we directly confront the variational solution (56) and FE solutions to two sets of representative experiments that confirm that this is in fact the case.

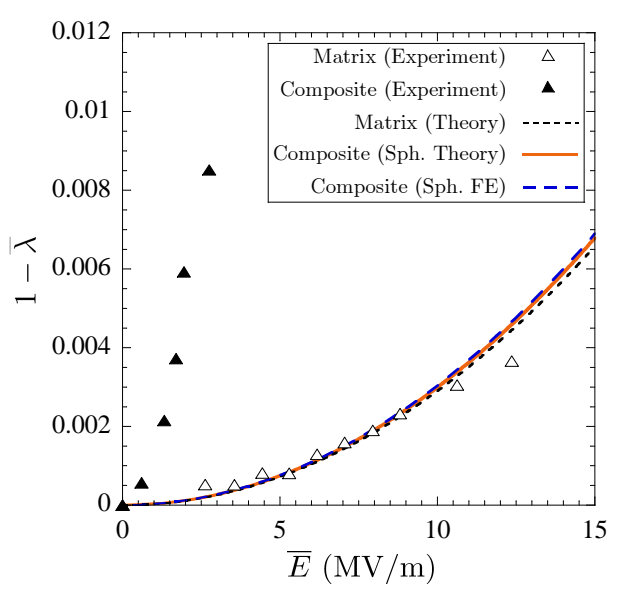

(a)

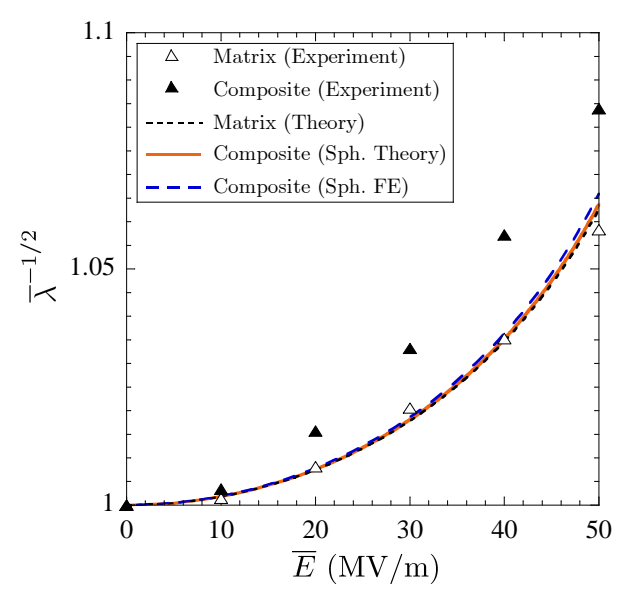

(b)

Figure 8: Comparisons between the theoretical predictions determined from the effective free-energy function (56) and the experimental data of Huang et al. (2005) and Liu et al. (2013) for the electrostriction of: (a) a polyurethane elastomer filled with semi-conducting o-CuPc particles at volume fraction $c=0.073$ and (b) a silicone elastomer filled with polarizationsaturating $\mathrm{TiO}_{2}$ particles at volume fraction $c=0.082$, under the application of a uniaxial electric field of magnitude $\bar{E}$. The experimental (theoretical) data for the dielectric elastomer composites is depicted as solid triangles (solid lines), while the experimental (theoretical) data for the underlying unfilled elastomers is depicted as hollow triangles (dotted lines). For further scrutiny, plots are also included (dashed lines) of corresponding FE predictions for the dielectric elastomer composites.

We begin by examining the experimental data of Huang et al. (2005) for the electrostriction response under a uniaxial electric field of a polyurethane elastomer filled with semi-conducting o-CuPc particles. The particles were reported to be roughly spherical in shape, about $40 \mathrm{~nm}$ in average diameter, and, with help of a polyacrylic acid (PAA) coating, well dispersed with overall isotropic symmetry. The total initial 
volume fraction of the o-CuPc particles and surrounding PAA coating was reported to be $c=0.073$. Figure 8(a) shows the measured electrostriction strain $\bar{\lambda}-1$ as a function of the applied electric field $\bar{E}$ for the filled polyurethane elastomer (solid triangles); see Section 4.3 for the definition of these variables. To aid the discussion, Fig. 8(a) also shows the measured electrostriction response for the unfilled polyurethane elastomer (empty triangles).

In computing the theoretical response predicted by the effective free-energy function (56), given the partial information available, we take the polyurethane elastomer in the composite to be characterized by the free-energy function (119) with parameters $\mu_{1}=0.410 \mathrm{MPa}, \mu_{2}=0.409 \mathrm{MPa}, \alpha_{1}=-8.034$, $\alpha_{2}=0.841$, and $\varepsilon=8.0 \varepsilon_{0}$, as fitted to the electrostriction and dielectric data provided by Huang et al. (2005) for the unfilled polyurethane elastomer ${ }^{4}$. Note that this assumes that the synthesis process in the presence of the o-CuPc nanoparticles does not alter the properties of the resulting polyurethane. We further assume that the particles are spherical in shape and monodisperse in size; recall from Section 6 that at the small volume fraction of particles $c=0.073$ of interest here, particle size dispersion has essentially no effect on macroscopic elastic dielectric properties. Moreover, since o-CuPc is a semi-conducting polymer with an initial shear modulus of about $1 \mathrm{GPa}$, we model the behavior of the particles by means of the freeenergy function (120) with material parameters $\mu_{\mathrm{p}}=1 \mathrm{GPa}, \varepsilon_{\mathrm{p}}=+\infty$, and $p_{s}=+\infty$. The electrostriction response that results from the effective free-energy function (56) given these inputs is displayed in Fig. 8(a) as a solid line. The corresponding FE prediction is also displayed in the figure as a dashed line. As expected from the sample results of subsection 6.2 , the theoretical results show little difference between the electrostriction of the dielectric elastomer composite and that of the unfilled polyurethane. This is in striking disagreement with the experimental data, which shows that the electrostriction of the dielectric elastomer composite is about 20 times larger than that of the unfilled polyurethane.

We now turn to examine the experimental data of Liu et al. (2013) for the electrostriction response under a uniaxial electric field of a silicone elastomer isotropically filled with roughly spherical $\mathrm{TiO}_{2}$ particles of about $3 \mu \mathrm{m}$ in average diameter at volume fraction $c=0.082$. Figure 8(b) shows the reported transverse (or so-called actuation) electrostriction $\bar{\lambda}^{-1 / 2}$ as a function of the applied electric field $\bar{E}$ for the filled silicone elastomer (solid triangles). Figure 8(b) also includes the electrostriction response for the unfilled silicone elastomer (empty triangles).

At the level of the variational solution (56), much like in the previous comparison, we take the silicone elastomer in the composite to be characterized by the free-energy function (119) with parameters $\mu_{1}=0.032 \mathrm{MPa}, \mu_{2}=0.023 \mathrm{MPa}, \alpha_{1}=3.837, \alpha_{2}=0.559$, and $\varepsilon=3.2 \varepsilon_{0}$, as fitted to the reported electrostriction and dielectric data for the unfilled silicone elastomer. Moreover, the particles are assumed to be spherical in shape and monodisperse in size. Given their polycrystalline rutile composition, we take their elastic dielectric behavior to be characterized by the free-energy function (120) with initial shear modulus $\mu_{\mathrm{p}}=110 \mathrm{GPa}$, initial permittivity $\varepsilon_{\mathrm{p}}=114 \varepsilon_{0}$, and polarization saturation $p_{s}=10^{-2} \mathrm{C} / \mathrm{m}^{2}$. The electrostriction response that results from the effective free-energy function (56) given these inputs is displayed in Fig. 8(b) as a solid line. For further scrutiny of the experimental data, the corresponding FE solution is also included (dashed line) in the figure. Again, as expected from the sample results of subsection 6.3, the theoretical predictions for the electrostriction response of the dielectric elastomer composite are practically indistinguishable from the response of the unfilled silicone elastomer. In stark contrast, the experimental data for the dielectric elastomer composite shows about a $50 \%$ enhancement in electrostriction with respect to the unfilled silicone.

The above glaring disagreement between the theoretical results and experiments makes it plain that the basic point of view (adopted throughout this work) that dielectric elastomer composites are two-phase particulate elastic dielectric composites is fundamentally incomplete, especially for cases involving stiff filler particles such as those shown in Fig. 8. In this regard, we recall that in elastomers filled with stiff particles the "anchoring" of the underlying polymer chains to the filler particles forces the chains into conformations that are very different from those in the bulk and that this results in "interphases" of different mechanical and physical behavior. The presence of such interphases (often referred to as bound rubber in the rubber science community) has long been known to have major effects on macroscopic properties when the filler particles are submicron in size (see, e.g., Leblanc, 2010; Goudarzi et al., 2015

\footnotetext{
${ }^{4}$ It is of note that the electrostriction data of Huang et al. (2005) imply a nonlinear elastic response of polyurethane that is about an order of magnitude softer than other experimental investigations have reported (see, e.g., Qi and Boyce, 2005).
} 
and references therein). Furthermore, free charges in such interphases may be present from the outset because of the fabrication process of the materials (see, e.g., Bauer et al., 2004; Lewis, 2004; Roy et al., 2005; Deng et al., 2014). They may also be injected from the particles upon the application of an electric field (see, e.g., Lewis, 2004; Roy et al., 2005). Whatever their origin, the presence of free interphasial charges has been recently shown to have the potential to lead to extreme enhancements of the macroscopic dielectric response of particulate composites and, by the same token, extreme enhancements of their electrostrictive response (Lopez-Pamies et al., 2014). We posit that the extreme enhancements in electromechanical properties that have been exhibited by emerging dielectric elastomer composites are the manifestation of the above-described interphasial phenomena ${ }^{5}$.
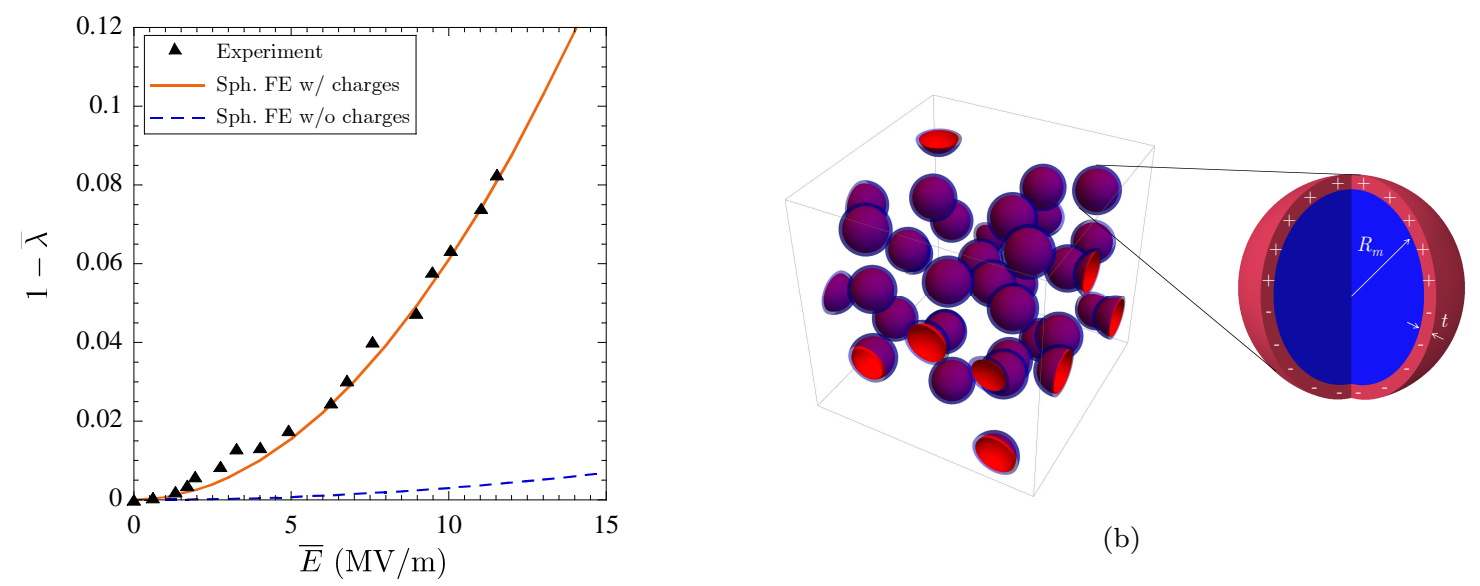

(b)

(a)

Figure 9: (a) Comparison between the experimental electrostriction data (solid triangles) of Huang et al. (2005) for a polyurethane elastomer filled with an isotropic distribution of semi-conducting o-CuPc particles at volume fraction $c=0.073$ and a theoretical prediction (solid line) featuring interphasial charges. In particular, the latter corresponds to the FE solution for the electrostriction response of the elastic dielectric composite whose defining unit cell is depicted in (b), namely, a nonGaussian dielectric elastomer (with free-energy function(119) and material parameters $\mu_{1}=0.410 \mathrm{MPa}, \mu_{2}=0.409 \mathrm{MPa}$, $\left.\alpha_{1}=-8.034, \alpha_{2}=0.841, \varepsilon=8.0 \varepsilon_{0}\right)$ filled with an isotropic distribution of rigid conducting monodisperse spherical particles at volume fraction $c=0.073$ that are surrounded by the distribution of free charges described by expression (121) with $R_{m}=20 \mathrm{~nm}, t=3 \mathrm{~nm}$, and $q_{i}=6500 \varepsilon_{0}$. The FE solution for the same elastic dielectric composite but without the interphasial charges (dashed line), already displayed in Fig. 8(a), is also included in this figure for comparison purposes.

By way of an example, in support of the above conjecture, Fig. 9(a) illustrates the close agreement possible between a theoretical prediction incorporating interphasial charges and the above-discussed experimental data of Huang et al. (2005) for a polyurethane elastomer filled with semi-conducting o-CuPc particles at volume fraction $c=0.073$. The theoretical prediction corresponds to the FE solution for the electrostriction response of a non-Gaussian dielectric elastomer filled with an isotropic distribution of monodisperse spherical particles that are surrounded by a layer of free charges. Specifically, like in the theoretical results presented in Fig. 8(a), the elastic dielectric behavior of the non-Gaussian dielectric elastomer is taken to be characterized by the free-energy function (119) with parameters $\mu_{1}=0.410 \mathrm{MPa}$, $\mu_{2}=0.409 \mathrm{MPa}, \alpha_{1}=-8.034, \alpha_{2}=0.841$, and $\varepsilon=8.0 \varepsilon_{0}$, as fitted to the electrostriction and dielectric data provided by Huang et al. (2005) for the unfilled polyurethane elastomer. The filler particles are taken to be rigid and electrically conducting. Finally, following Lopez-Pamies et al. (2014), the distribution of free charges that surround the particles is taken to be characterized by the charge density (per unit

\footnotetext{
${ }^{5}$ While it is known that the elastomeric matrix material in a given filled elastomer may not have the same mechanical and physical properties as the "same" elastomer synthesized in the absence of filler particles, the expected range of possible differences cannot account for the extreme enhancements observed experimentally. Viscous and dielectric dissipative phenomena might also contribute to the observed enhancements. Preliminary calculations in the context of time-dependent dielectric composite materials have suggested, however, that they are of lesser importance than interphasial phenomena (Ladeb and Lopez-Pamies, 2015).
} 
undeformed volume)

$$
Q(\mathbf{X})=\theta_{i}(\mathbf{X}) q_{i} \frac{\overline{\mathbf{E}} \cdot\left(\mathbf{X}-\mathbf{X}^{c}\right)}{R_{m}\left|\mathbf{X}-\mathbf{X}^{c}\right|} \quad \text { with } \quad \theta_{i}(\mathbf{X})= \begin{cases}1 & \text { if } R_{m}<\left|\mathbf{X}-\mathbf{X}^{c}\right|<R_{m}+t \\ 0 & \text { otherwise }\end{cases}
$$

Here, $R_{m}$ and $\mathbf{X}^{c}$ stand for the common radius and the position vector of the center of each spherical particle, $t$ denotes the common constant thickness of the spatial regions around each particle where the charges are contained, and $q_{i}$ is a constant of choice (of units $\mathrm{F} / \mathrm{m}$ ) that physically can be viewed as a measure of charge content. The FE result presented in Fig. 9(a) corresponds to the values $R_{m}=20 \mathrm{~nm}$, $t=3 \mathrm{~nm}$, and $q_{i}=6500 \varepsilon_{0}$. Figure $9(\mathrm{~b})$ illustrates the unit cell whose periodic repetition defines the precise microstructure in this example.

We emphasize that expression (121) is an ad hoc constitutive choice and that little is actually known about interphasial charges in dielectric elastomer composites. Further studies in this direction appear to be of the essence in order to understand the fundamental microscopic mechanisms behind the remarkable electromechanical properties that dielectric elastomer composites seem capable of achieving.

\section{Acknowledgements}

Support for this work by the National Science Foundation through the CAREER Grant CMMI-1219336 (formerly CMMI-1055528) is gratefully acknowledged.

\section{Appendix. A conforming FE approximation based on Crouzeix-Raviart-type elements}

In this appendix, we provide details of the conforming FE method that we employ to construct numerical solutions for the Euler-Lagrange equations (116). Seeking the ability to discretize complex domains, we consider partitions of the unit cell that comprise $N_{e}$ non-overlapping "curved" tetrahedral elements: $Y=\bigcup_{e=1}^{N_{e}} \mathcal{E}^{(e)}$ with $\mathcal{E}^{(i)} \cap \mathcal{E}^{(j)}=\varnothing \forall i \neq j$. Specifically, each element $e$ possesses 15 nodes with coordinates $\left(X_{1}^{(e, m)}, X_{2}^{(e, m)}, X_{3}^{(e, m)}\right), m=1, \ldots, 15$. In terms of these nodal coordinates, with help of the reference tetrahedral element $\mathcal{T}=\{(\xi, \eta, \rho): 0 \leq \xi, \eta, \rho \leq 1, \xi+\eta+\rho \leq 1\}$, the domain occupied by each element $e$ is defined parametrically by $\mathcal{E}^{(e)}=\left\{\left(X_{1}, X_{2}, X_{3}\right): X_{i}=\mathcal{M}(\xi, \eta, \rho)=\sum_{m=1}^{10} N_{Q}^{(m)}(\xi, \eta, \rho) X_{i}^{(e, m)},(\xi, \eta, \rho) \in\right.$ $\mathcal{T}$ \}. Figure 10 illustrates a generic element $\mathcal{E}^{(e)}$ and its mapping from the reference element $\mathcal{T}$ indicating the location of all of its 15 nodes. In the above-introduced parametric mapping $\mathcal{M}, N_{Q}^{(m)}(\xi, \eta, \rho)$ stand for the shape functions associated with the classical 10-node tetrahedral element. For convenience, we recall them here:

$$
\begin{aligned}
& N_{Q}^{(1)}(\xi, \eta, \rho)=\xi(2 \xi-1), \quad N_{Q}^{(2)}(\xi, \eta, \rho)=\eta(2 \eta-1), \\
& N_{Q}^{(3)}(\xi, \eta, \rho)=(1-\xi-\eta-\rho)(1-2 \xi-2 \eta-2 \rho), \quad N_{Q}^{(4)}(\xi, \eta, \rho)=\rho(2 \rho-1), \\
& N_{Q}^{(5)}(\xi, \eta, \rho)=4 \xi \eta, \quad N_{Q}^{(6)}(\xi, \eta, \rho)=4 \eta(1-\xi-\eta-\rho), \quad N_{Q}^{(7)}(\xi, \eta, \rho)=4 \xi(1-\xi-\eta-\rho), \\
& N_{Q}^{(8)}(\xi, \eta, \rho)=4 \xi \rho, \quad N_{Q}^{(9)}(\xi, \eta, \rho)=4 \eta \rho, \quad N_{Q}^{(10)}(\xi, \eta, \rho)=4 \rho(1-\xi-\eta-\rho) .
\end{aligned}
$$



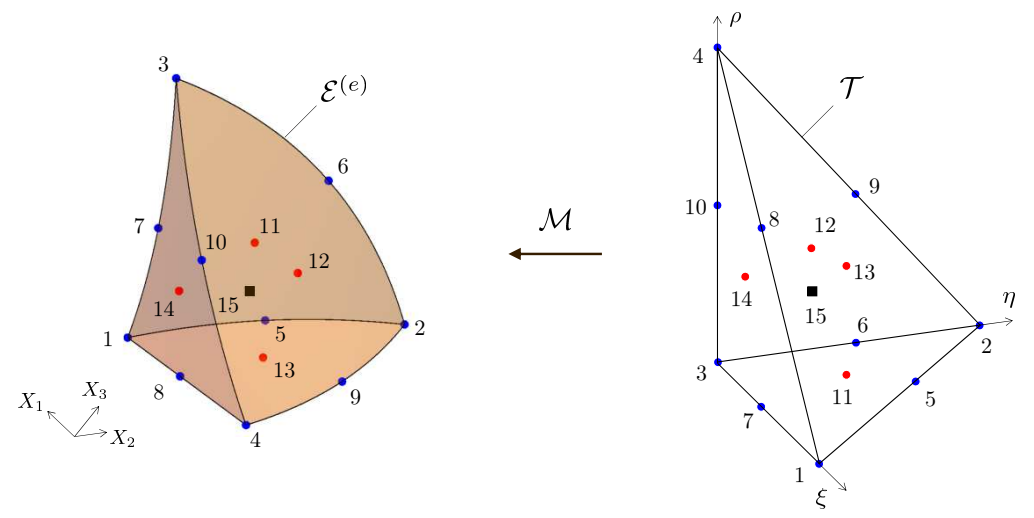

Figure 10: Schematic of a "curved" tetrahedral element occupying a domain $\mathcal{E}^{(e)} \subseteq Y$ and its mapping $\mathcal{M}$ from the reference element $\mathcal{T}$. Blue circles indicate nodes 1 through 10. They are located at the four vertices and the six mid-edge points of $\mathcal{T}$. Nodes 11 through 14, which are located at the four mid-face points of $\mathcal{T}$, are shown as red circles. Node 15 , located at the barycenter of $\mathcal{T}$, is indicated by a black square.

Having defined the partition of the unit cell $Y$, we are now in a position to define $\mathcal{U}_{h}, \mathcal{F}_{h}$, and $\mathcal{P}_{h}$, the finite dimensional subspaces of $\mathcal{U}, \mathcal{F}$, and $\mathcal{P}$. They read as follows:

$$
\begin{aligned}
& \mathcal{U}^{h}=\left\{\mathbf{u}^{h} \in\left[\mathcal{C}^{0}(Y)\right]^{3} \cap \mathcal{U}:\left.u_{i}^{h}\left(X_{1}, X_{2}, X_{3}\right)\right|_{\mathcal{E}^{(e)}}=\sum_{m=1}^{15} N_{C R}^{(m)}(\xi, \eta, \rho) u_{i}^{(e, m)}, \forall e=1, \ldots, N_{e}\right\}, \\
& \mathcal{F}^{h}=\left\{\Phi^{h} \in \mathcal{C}^{0}(Y) \cap \mathcal{F}:\left.\Phi^{h}\left(X_{1}, X_{2}, X_{3}\right)\right|_{\mathcal{E}^{(e)}}=\sum_{m=1}^{15} N_{C R}^{(m)}(\xi, \eta, \rho) \Phi^{(e, m)}, \forall e=1, \ldots, N_{e}\right\} \\
& \mathcal{P}^{h}=\left\{p^{h} \in \mathcal{P}:\left.p^{h}\left(X_{1}, X_{2}, X_{3}\right)\right|_{\mathcal{E}(e)}=\sum_{k=0}^{3} N_{P}^{(k)}(\xi, \eta, \rho) p^{(e, k)}, \forall e=1, \ldots, N_{e}\right\} .
\end{aligned}
$$

Here, $\mathcal{C}^{0}(Y)$ stands for the set of continuous functions defined on $Y, u_{i}^{(e, m)}$ and $\Phi^{(e, m)}$ denote the component $i$ of the displacement and the electric potential at node $m$ of element $e$, while $p^{(e, k)}$ denote the value $(k=0)$ of the pressure field and the three components $(k=1,2,3)$ of its gradient at node 15 of the domain $\mathcal{E}^{(e)}$. In the above expressions, $N_{C R}^{(m)}(\xi, \eta, \rho)$ stand for the shape functions

$N_{C R}^{(1)}(\xi, \eta, \rho)=3 \xi[\eta \rho+(\eta+\rho)(1-\eta-\xi-\rho)]+\xi[2 \xi-1-4 \eta \rho(1-\eta-\xi-\rho)]$,

$N_{C R}^{(2)}(\xi, \eta, \rho)=3 \eta[\xi \rho+(\xi+\rho)(1-\eta-\xi-\rho)]+\eta[2 \eta-1-4 \xi \rho(1-\eta-\xi-\rho)]$,

$N_{C R}^{(3)}(\xi, \eta, \rho)=3(1-\eta-\xi-\rho)(\eta \xi+\eta \rho+\xi \rho)+(1-\eta-\xi-\rho)[1-2 \eta-2 \xi-2 \rho-4 \eta \xi \rho]$,

$N_{C R}^{(4)}(\xi, \eta, \rho)=3 \rho[\eta \xi+(\eta+\xi)(1-\eta-\xi-\rho)]+\rho[2 \rho-1-4 \eta \xi(1-\eta-\xi-\rho)]$,

$N_{C R}^{(5)}(\xi, \eta, \rho)=4 \eta \xi[8 \rho(1-\eta-\xi-\rho)-3(1-\eta-\xi)+1], \quad N_{C R}^{(6)}(\xi, \eta, \rho)=4 \eta(1-\eta-\xi-\rho)[8 \xi \rho-3(\xi+\rho)+1]$,

$N_{C R}^{(7)}(\xi, \eta, \rho)=4 \xi(1-\eta-\xi-\rho)[8 \eta \rho-3(\eta+\rho)+1], \quad N_{C R}^{(8)}(\xi, \eta, \rho)=4 \xi \rho[8 \eta(1-\eta-\xi-\rho)-3(1-\xi-\rho)+1]$,

$N_{C R}^{(9)}(\xi, \eta, \rho)=4 \eta \rho[8 \xi(1-\eta-\xi-\rho)-3(1-\eta-\rho)+1], \quad N_{C R}^{(10)}(\xi, \eta, \rho)=4 \rho(1-\eta-\xi-\rho)[8 \eta \xi-3(\eta+\xi)+1]$,

$N_{C R}^{(11)}(\xi, \eta, \rho)=27 \eta \xi(1-4 \rho)(1-\eta-\xi-\rho), \quad N_{C R}^{(12)}(\xi, \eta, \rho)=27 \eta \xi \rho(4 \eta+4 \xi+4 \rho-3)$,

$N_{C R}^{(13)}(\xi, \eta, \rho)=27 \eta \rho(1-4 \xi)(1-\eta-\xi-\rho), \quad N_{C R}^{(14)}(\xi, \eta, \rho)=27 \xi \rho(1-4 \eta)(1-\eta-\xi-\rho)$,

$N_{C R}^{(15)}(\xi, \eta, \rho)=256 \eta \xi \rho(1-\eta-\xi-\rho)$,

while $N_{P}^{(k)}(\xi, \eta, \rho)$ are given by $(125)$.

$$
N_{P}^{(0)}(\xi, \eta, \rho)=1, \quad N_{P}^{(1)}(\xi, \eta, \rho)=\xi-\frac{1}{4}, \quad N_{P}^{(2)}(\xi, \eta, \rho)=\eta-\frac{1}{4}, \quad N_{P}^{(3)}(\xi, \eta, \rho)=\rho-\frac{1}{4} .
$$


Again, the parameters $\xi, \eta, \rho$ in expressions (123)-(125) are related to the physical coordinates $X_{1}, X_{2}$, $X_{3}$ through the mapping $\mathcal{M}$.

At this stage, it is fitting to mention that the finite dimensional subspaces $(123)_{1}$ and $(123)_{3}$ for the displacement field $\mathbf{u}$ and the pressure field $p$ are three-dimensional analogues (see, e.g., Chapter II in Girault and Raviart, 1986; Chapter 8 in Boffi et al., 2012) of finite dimensional subspaces originally introduced in the context of two-dimensional Stokes flow by Crouzeix and Raviart (1973), thus the $C R$ subscript in (124). They have the merit to have been proven to lead to stable and convergent formulations in the context of isotropic linear elasticity, irrespectively of the compressibility of the medium (Chapter II in Girault and Raviart, 1986; Chapter 8 in Boffi et al., 2012). Moreover, in the context of nonlinear pdes, our own numerical investigations and those of others for different types of nonlinear pdes (see, e.g., Van de Vosse et al., 2003) have shown that the finite dimensional subspaces (123) lead to formulations with the expected convergence rates, namely, in the present context, $\left\|\mathbf{u}^{h}-\mathbf{u}\right\|_{L^{2}}=O\left(h^{3}\right),\left\|\Phi^{h}-\Phi\right\|_{L^{2}}=O\left(h^{3}\right)$, $\left\|p^{h}-p\right\|_{L^{2}}=O\left(h^{2}\right)$ with $h=\max \operatorname{diam}\left\{\mathcal{E}^{(e)}\right\}$.

Standard assembly procedures allows one to construct global shape functions $N_{C R}^{h(n)}(\mathbf{X}), n=1, \ldots, N_{n}$, and $N_{P}^{h(l)}(\mathbf{X}), l=0, \ldots, 4 N_{e}-1$, so that the displacement $\mathbf{u}^{h}$ and the electric potential $\Phi^{h}$ continuous trial fields, and the pressure $p^{h}$ discontinuous trial field in (123) can be rewritten in the global form

$$
u_{i}^{h}(\mathbf{X})=\sum_{n=1}^{N_{n}} u_{i}^{(n)} N_{C R}^{h(n)}(\mathbf{X}), \quad \Phi^{h}(\mathbf{X})=\sum_{n=1}^{N_{n}} \Phi^{(n)} N_{C R}^{h(n)}(\mathbf{X}), \quad \text { and } \quad p^{h}(\mathbf{X})=\sum_{l=0}^{4 N_{e}-1} p^{(l)} N_{P}^{h(l)}(\mathbf{X}),
$$

where the global degrees of freedom $u_{i}^{(n)}$ and $\Phi^{(n)}$ correspond physically to the component $i$ of the displacement $\mathbf{u}^{h}$ and the electric potential $\Phi^{h}$ at node $n$, while $p^{(l)}$ correspond physically to the value $(l \equiv 0 \bmod 4)$ of the pressure field $p^{h}$ and the three components $(l \equiv 1,2,3 \bmod 4)$ of its gradient at the barycenter of $\mathcal{E}^{(e)}, e=\lfloor l / 4\rfloor+1, l \neq \equiv 0 \bmod 4$; here, $\lfloor x\rfloor \doteq \max \{z \in \mathbb{Z}: z \leq x\}$ denotes the floor function. Similarly, the test functions $\mathbf{v}^{h} \in \mathcal{U}^{h} \cap \mathcal{U}^{0}, \psi^{h} \in \mathcal{F}^{h} \cap \mathcal{F}^{0}$, and $q^{h} \in \mathcal{P}^{h}$ can be written in global form as

$$
v_{i}^{h}(\mathbf{X})=\sum_{n=1}^{N_{n}} v_{i}^{(n)} N_{C R}^{h(n)}(\mathbf{X}), \quad \psi^{h}(\mathbf{X})=\sum_{n=1}^{N_{n}} \psi^{(n)} N_{C R}^{h(n)}(\mathbf{X}), \quad \text { and } \quad q^{h}(\mathbf{X})=\sum_{l=0}^{4 N_{e}-1} q^{(l)} N_{P}^{h(l)}(\mathbf{X}) .
$$

In the above expressions, $N_{n}$ stands for the total number of nodes in the partition of the cubic unit cell $Y$. Their location must be selected so that the intersections of any two opposite faces of the partition of $Y$ are identical. This geometric constraint is needed to be able to consistently enforce the required periodicity conditions (implied by relations (115) 1-2) of the continuous trial fields $\mathbf{u}^{h}$ and $\Phi^{h}$ and the Y-periodicity of the continuous test functions $\mathbf{v}^{h}$ and $\psi^{h}$. In light of this constraint, it proves useful to define the subsets of interior-node numbers I, vertex-node numbers V, face-node numbers F, and edge-node numbers $\mathrm{E}$ that make up the entire set of nodes. They read as

$$
\begin{aligned}
\mathrm{I}= & \left\{n: X_{i}^{(n)} \in(0,1) \forall i \in\{1,2,3\}\right\}, \quad \mathrm{V}=\left\{n: X_{i}^{(n)} \in\{0,1\} \forall i \in\{1,2,3\}\right\}, \\
\mathrm{F}= & \left\{(n, p): X_{j}^{(n)}=X_{j}^{(p)} \in(0,1), \quad X_{k}^{(n)}=X_{k}^{(p)} \in(0,1), \quad X_{i}^{(n)}=0, \quad X_{i}^{(p)}=1 \forall(i, j, k): \epsilon_{i j k}=1\right\}, \\
\mathrm{E}=\{ & \left\{(n, p, q, r): X_{i}^{(n)}=X_{i}^{(p)}=X_{i}^{(q)}=X_{i}^{(r)} \in(0,1), \quad X_{j}^{(n)}=X_{k}^{(n)}=X_{k}^{(p)}=X_{j}^{(q)}=0,\right. \\
& \left.X_{j}^{(p)}=X_{k}^{(q)}=X_{k}^{(r)}=X_{j}^{(r)}=1 \forall(i, j, k): \epsilon_{i j k}=1\right\},
\end{aligned}
$$

where $\epsilon_{i j k}$ stands for the three-dimensional permutation symbol.

Direct use of the trial fields (126) and the test functions (127) in the Euler-Lagrange equations (116) 
leads to the following system of non-linear algebraic equations

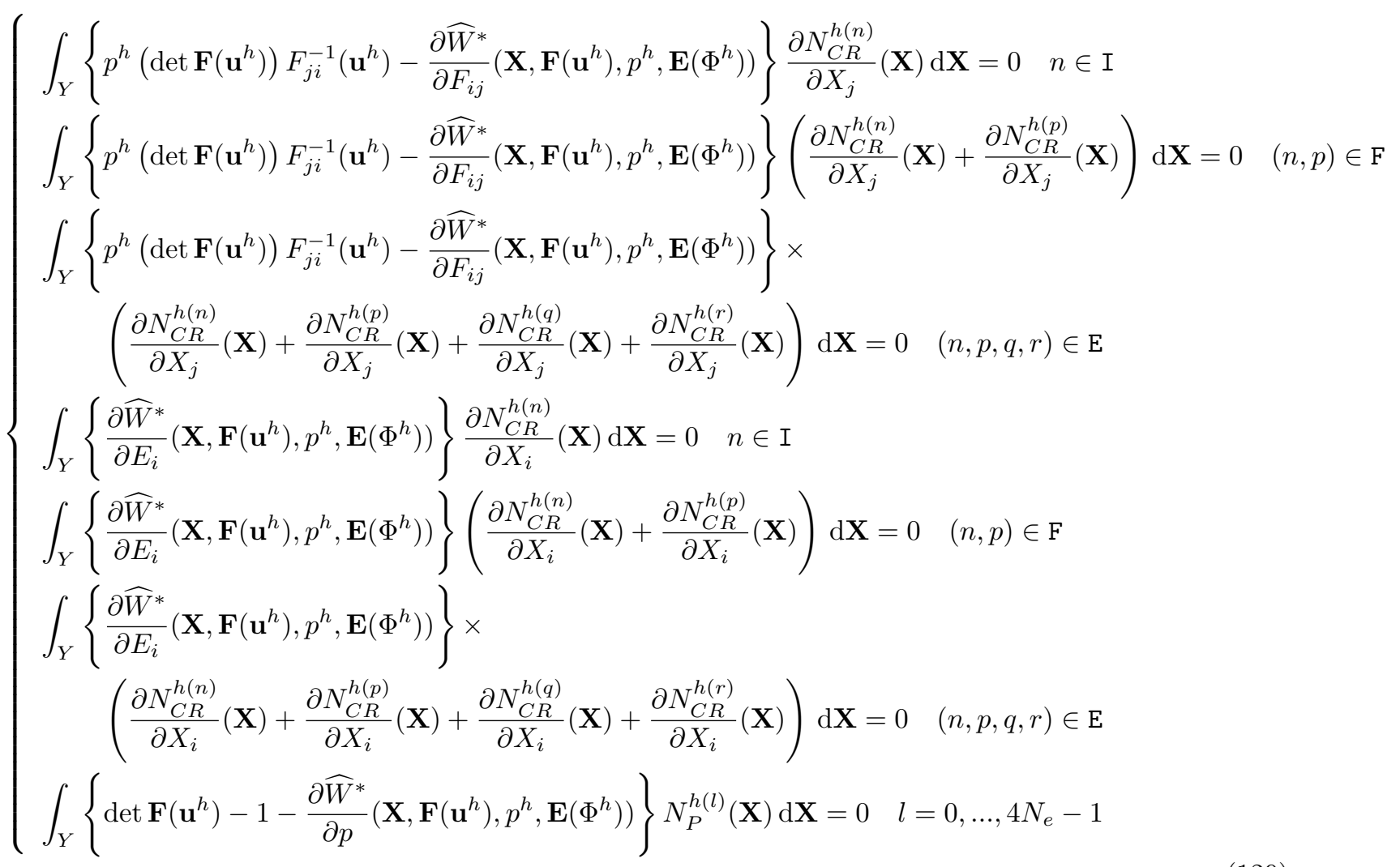

for the global degrees of freedom $u_{i}^{(n)}, \Phi^{(n)}, p^{(l)}$ for $n \notin \mathrm{V}$, together with the periodicity constraints

$$
\begin{aligned}
& u_{i}^{h}\left(1, X_{2}, X_{3}\right)-u_{i}^{h}\left(0, X_{2}, X_{3}\right)=\left(\bar{F}_{i 1}-\delta_{i 1}\right), \\
& u_{i}^{h}\left(X_{1}, 1, X_{3}\right)-u_{i}^{h}\left(X_{1}, 0, X_{3}\right)=\left(\bar{F}_{i 2}-\delta_{i 2}\right), \\
& u_{i}^{h}\left(X_{1}, X_{2}, 1\right)-u_{i}^{h}\left(X_{1}, X_{2}, 0\right)=\left(\bar{F}_{i 3}-\delta_{i 3}\right), \\
& \Phi^{h}\left(1, X_{2}, X_{3}\right)-\Phi^{h}\left(0, X_{2}, X_{3}\right)=-\bar{E}_{1}, \\
& \Phi^{h}\left(X_{1}, 1, X_{3}\right)-\Phi^{h}\left(X_{1}, 0, X_{3}\right)=-\bar{E}_{2}, \\
& \Phi^{h}\left(X_{1}, X_{2}, 1\right)-\Phi^{h}\left(X_{1}, X_{2}, 0\right)=-\bar{E}_{3},
\end{aligned}
$$

and known values at the vertices

$$
u_{i}^{(n)}=\left(\bar{F}_{i j}-\delta_{i j}\right) X_{j}^{(n)} \quad \text { and } \quad \Phi^{(n)}=-\bar{E}_{i} X_{i}^{(n)} \quad \text { for } n \in \mathrm{V} .
$$

Having computed the values of all the degrees of freedom $u_{i}^{(n)}, \Phi^{(n)}, p^{(l)}$ from the set of algebraic equations (129)-(131) for a given discretization of $Y$, given local free-energy function $W(\mathbf{X}, \mathbf{F}, \mathbf{E})$, and given electromechanical loading $\overline{\mathbf{F}}$ and $\overline{\mathbf{E}}$, the pointwise displacement field $\mathbf{u}^{h}(\mathbf{X})$, electric potential $\Phi^{h}(\mathbf{X})$, and pressure field $p^{h}(\mathbf{X})$ can be readily determined form expressions (126). In turn, these fields can be readily utilized to compute the effective free-energy function (114) for the dielectric elastomer composite of interest. Here, it is important to recall that the elastic dielectric response of dielectric elastomer composites may exhibit electromechanical limit loads. In order to be able to compute solutions past beyond eventual electromechanical limit loads, we solve the system of nonlinear algebraic equations (129)-(131) by means of an arc-length method. In particular, while there are many possibilities available, we found the use of the standard constraint condition put forward by Crisfield (1981) to be adequate for our purposes here. 


\section{References}

[1] Bauer, S., Gerhard-Multhaupt, R., Sessler, G.M., 2004. Ferroelectrets: Soft electroactive foams for transducers. Physics Today 57, 37-43.

[2] Boffi, D., Brezzi, F., Fortin, M., 2012. Mixed Finite Element Methods and Applications. Springer

[3] Carpi, F., De Rossi, D., 2005. Improvement of electromechanical actuating performances of a silicone dielectric elastomer by dispersion of titanium dioxide powder. IEEE Transactions on Dielectrics and Electrical Insulation 12, 835-843.

[4] Chi, H., Talischi, C., Lopez-Pamies, O., Paulino, G.H., 2014. Polygonal finite elements for finite elasticity. International Journal for Numerical Methods in Engineering 101, 305-328.

[5] Crisfield, M.A., 1981. A fast incremental-iterative solution procedure that handles "snap-through". Computers and Structures 13, 55-62.

[6] Crouzeix, M., Raviart, P.-A., 1973. Conforming and nonconforming finite element methods for solving the stationary Stokes equations. R.A.I.R.O. R3, 33-76.

[7] deBotton, G., Shmuel, G., 2010. A new variational estimate for the effective response of hyperelastic composites. J. Mech. Phys. Solids 58, 466-483.

[8] Debye, P.J.W., 1929. Polar Molecules. The Chemical Catalog Company, New York.

[9] Deng, Q., Liu, L., Sharma, P., 2014. Electrets in soft materials: Nonlinearity, size effects, and giant electromechanical coupling. Physical Review E 90, 012603.

[10] Fassler, A., Majidi, C., 2015. Liquid-phase metal inclusions for a conductive polymer composite. Adv. Mater. 27, 19281932.

[11] Geymonat, G., Müller, S., Triantafyllidis, N., 1993. Homogenization of nonlinearly elastic materials, microscopic bifurcation and macroscopic loss of rank-one convexity. Arch. Ration. Mech. Anal. 122, 231-290.

[12] Girault, V., Raviart, P.-A., 1986. Finite Element Methods for Navier-Stokes Equations. Theory and Algorithms. Springer.

[13] Goudarzi, T., Spring, D.W., Paulino, G.H., Lopez-Pamies, O. 2015. Filled elastomers: A theory of filler reinforcement based on hydrodynamic and interphasial effects. Journal of the Mechanics and Physics of Solids 80, 37-67.

[14] Huang, C., Zhang, Q.M., 2004. Enhanced dielectric and electromechanical response in high-dielectric constant allpolymer percolative composites. Advanced Functional Materials 14, 501-506.

[15] Huang, C., Zhang, Q.M., Li, J.Y., Rabeony, M., 2005. Colossal dielectric and electromechanical responses in selfassembled polymeric nanocomposites. Applied Physics Letters 87, 182901.

[16] Keip, M.A., Steinmann, P., Schröder, J., 2014. Two-scale computational homogenization of electro-elasticity at finite strains. Computer Methods in Applied Mechanics and Engineering 278, 62-79.

[17] Ladeb, M., Lopez-Pamies, O., 2015. Extreme enhancement and reduction of the time-dependent dielectric response of polymer nanoparticulate composites via interphasial charges. Unpublished work.

[18] Lahellec, N., Mazerolle, F., Michel, J.-C., 2004. Second-order estimate of the macroscopic behavior of periodic hyperelastic composites: theory and experimental validation. J. Mech. Phys. Solids 52, 27-49.

[19] Langevin, P., 1905. Sur la theorie du magnetisme. J. Phys. Theor. Appl. 4, 678-693.

[20] Leblanc, J.L., 2010. Filled Polymers: Science and Industrial Applications. CRC Press, Boca Raton.

[21] Lefèvre, V., Lopez-Pamies, O. 2016. Nonlinear electroelastic deformations of dielectric elastomer composites: I — Ideal elastic dielectrics. Submitted.

[22] Lewis, T.J., 2004. Interfaces are the dominant feature of dielectrics at the nanometric level. IEEE Transactions on Dielectrics and Electrical Insulation 11, 739-753.

[23] Li, J.Y., 2003. Exchange coupling in p(VDF-TRFE)copolymer based all-organic composites with giant electrostriction. Phys. Rev. Lett. 90, 217601-217604.

[24] Li, J.Y., Huang, C., Zhang, Q., 2004. Enhanced electromechanical properties in all-polymer percolative composites. Appl. Phys. Lett. 84, 3124.

[25] Li, W., Landis, C.M., 2012. Deformation and instabilities in dielectric elastomer composites. Smart Mater. Struct. 21, 094006

[26] Liu, H., Zhang, L., Yang, D., Yu, Y., Yao, L., Tian, M., 2013. Mechanical, dielectric, and actuated strain of silicone elastomer filled with various types of $\mathrm{TiO}_{2}$. Soft Materials 11, 363-370.

[27] Lopez-Pamies, O., 2010. A new $I_{1}$-based hyperelastic model for rubber elastic materials. C. R. Mec. 338, 3-11.

[28] Lopez-Pamies, O., 2014. Elastic dielectric composites: Theory and application to particle-filled ideal dielectrics. J. Mech. Phys. Solids 64, 61-82.

[29] Lopez-Pamies, O., Goudarzi, T., Danas, K. 2013. The nonlinear elastic response of suspensions of rigid inclusions in rubber: II - A simple explicit approximation for finite-concentration suspensions. J. Mech. Phys. Solids 61, $19-37$.

[30] Lopez-Pamies, O., Goudarzi, T., Meddeb, A.B., Ounaies, Z., 2014. Extreme enhancement and reduction of the dielectric response of polymer nanoparticulate composites via interphasial charges. Applied Physics Letters $104,242904$.

[31] McCarthy, D.N., Risse, S., Katekomol, P., Kofod, G., 2009. The effect of dispersion on the increased relative permittivity of $\mathrm{TiO}_{2}$ /SEBS composites. J. Phys. D: Appl. Phys. 42, 145406.

[32] Meddeb, A.M., Ounaies, Z., 2012. Nano-enhanced polymer composites for energy storage applications. Proc. SPIE 8342, 834207.

[33] Michel, J.C., Lopez-Pamies, O., Ponte Castañeda, P., Triantafyllidis, N., 2010. Microscopic and macroscopic instabilities in finitely strained fiber-reinforced elastomers. Journal of the Mechanics and Physics of Solids 58, $1776-1803$.

[34] Miehe, C. Vallicotti, D., Teichtmeister, S., 2016. Homogenization and multiscale stability analysis in finite magnetoelectro-elasticity. Application to soft matter EE, ME and MEE composites. Comput. Methods Appl. Mech. Engrg. 300, $294-346$.

[35] Ponte Castañeda, P., 1991. The effective mechanical properties of nonlinear isotropic composites. J. Mech. Phys. Solids $39,45-71$. 
[36] Qi, H.J., Boyce, M.C., 2005. Stress-strain behavior of thermoplastic polyurethanes. Mechanics of Materials 37, 817-839.

[37] Roy, M., Nelson, J.K., MacCrone, R.K., Schadler, L.S., Reed, C.W., Keefe, R., Zenger, W., 2005. Polymer nanocomposites dielectrics - The role of the interface. IEEE Transactions on Dielectrics and Electrical Insulation 12, 629-643.

[38] Spinelli, S.A., Lefèvre, V., Lopez-Pamies, O., 2015. Dielectric elastomer composites: A general closed-form solution in the small-deformation limit. J. Mech. Phys. Solids 83, 263-284.

[39] Talbot, D.R.S., Willis, J.R., 1985. Variational principles for inhomogeneous nonlinear media. IMA J. Appl. Math. 35, 3954.

[40] Tian, L., Tevet-Deree, L., deBotton, G., Bhattacharya, K., 2012. Dielectric elastomer composites. J. Mech. Phys. Solids 60, 181-198.

[41] Van de Vosse, F., De Hart, J., Van Oijen, C., Bessems, D., Gunther, T., Segal, A., Wolters, B., Stijnen, J., Baaijens., F., 2003. Finite-element-based computational methods for cardiovascular fluid-structure interaction. J. Eng. Math. 47, $335-368$.

[42] Willis, J.R., 1991. On methods for bounding the overall properties of nonlinear composites. J. Mech. Phys. Solids 39, $73-86$.

[43] Wissler, M.T., 2007. Modeling Dielectric Elastomer Actuators (Ph.D. dissertation). Eidgenössische Technische Hochschule Zürich. Switzerland.

[44] Zhang, Q.M., Hengfeng, L., Poh, M., Xia, F., Cheng, Z.-Y., Xu, H., Huang, C., 2002. An all-organic composite actuator material with high dielectric constant. Nature 419, 284-287. 